\title{
Incorporating physical constraints in braneworld black-string solutions for a Minkowski brane in scalar-tensor gravity
}

\author{
Theodoros Nakas $\oplus^{1, *}$ Panagiota Kanti® ${ }^{1, \dagger}$ and Nikolaos Pappas $\oplus^{2, \$}$ \\ ${ }^{1}$ Division of Theoretical Physics, Department of Physics, University of Ioannina, \\ Ioannina GR-45110, Greece \\ ${ }^{2}$ Nuclear and Particle Physics Section, Physics Department, \\ National and Kapodistrian University of Athens, Athens GR-15771, Greece
}

(Received 29 January 2020; accepted 3 April 2020; published 27 April 2020)

\begin{abstract}
In the framework of a general scalar-tensor theory, where the scalar field is nonminimally coupled to the five-dimensional Ricci scalar curvature, we investigate the emergence of complete braneworld solutions. By assuming a variety of forms for the coupling function, we solve the field equations in the bulk, and determine in an analytic way the form of the gravitational background and scalar field in each case. The solutions are always characterized by a regular scalar field, a finite energy-momentum tensor, and an exponentially decaying warp factor even in the absence of a negative bulk cosmological constant. The space-time on the brane is described by the Schwarzschild solution leading to either a nonhomogeneous black-string solution in the bulk, when the mass parameter $M$ is nonzero, or a regular anti-de Sitter spacetime, when $M=0$. We construct physically acceptable solutions by demanding in addition a positive effective gravitational constant on our brane, a positive total energy density for our brane, and the validity of the weak energy condition in the bulk. We find that, although the theory does not allow for all three conditions to be simultaneously satisfied, a plethora of solutions emerge which satisfy the first two, and most fundamental, conditions.
\end{abstract}

DOI: 10.1103/PhysRevD.101.084056

\section{INTRODUCTION}

The first higher-dimensional formulation of the general theory of relativity [1-3] by Kaluza [4] and Klein [5] is almost as old as the original theory itself. In the 1980s, the postulation of the existence of extra spacelike dimensions in nature was combined with the string-inspired notion of the brane, which plays the role of our four-dimensional world [6,7]. At the turn of the last century, the modern braneworld theories were proposed [8-12] in which the extra spatial dimensions may be large compared to the Planck scale or, even, infinite. This radical change in the structure and topology of space-time has significantly affected the properties of all gravitational solutions which emerge in the framework of the new theories. In addition, the phase space of solutions of a higher-dimensional gravitational theory now contains a variety of black objects,

\footnotetext{
t.nakas@uoi.gr

pkanti@uoi.gr

*npappas@uoi.gr
}

Published by the American Physical Society under the terms of the Creative Commons Attribution 4.0 International license. Further distribution of this work must maintain attribution to the author(s) and the published article's title, journal citation, and DOI. Funded by SCOAP ${ }^{3}$. namely black holes, black strings, black branes, black rings, or black saturns [13].

The warped braneworld model $[11,12]$ admits an infinitely long extra dimension which is nevertheless accompanied by the localization of the graviton close to our brane. This is realized with the help of an exponentially decreasing warp factor in the expression of the line element which describes the higher-dimensional gravitational background. However, the presence of this factor has proven to be an insurmountable obstacle in the derivation of an analytical, nonapproximate solution describing a regular, localized close-to-our-brane black hole (see Refs. [14-46] for an impartial list of works on this topic; for a number of numerical solutions describing in principle regular braneworld black holes, see [47-53]).

The aforementioned attempts to derive an analytical solution of a localized braneworld black hole have in fact proven that solutions describing a different type of a black object, namely a black string, are much easier to construct. Although the first such solution [14] in the context of the warped braneworld models [11,12] was proven to be unstable [54,55], a variety of higher-dimensional black strings have since been derived in the context of different theories in the literature-see, for example Refs. [56-74]. In $[31,38]$, a braneworld model, that contained a bulk scalar field with an arbitrary potential and a nonminimal coupling 
to gravity, was studied. Scalar-tensor theories of this type are very popular and have been extensively studied in the context of four-dimensional gravity, while braneworld generalizations have been studied in the literature before, both in static and nonstatic backgrounds [75-80]. The objective of the analyses in [31,38] was to derive an analytical solution describing a regular, localized black hole; although no such solution was found, these studies hinted that black-string solutions were in fact much easier to emerge in the context of a nonminimally coupled scalartensor braneworld model.

To demonstrate this, in [69] we launched a comprehensive study of the types of black-string solutions that emerge in the context of this theory. Solving analytically the complete set of gravitational and scalar-field equations in the bulk, we determined novel black-string solutions which reduced to a Schwarzschild-(anti-)de Sitter space-time on the brane. The sign of the effective cosmological constant on the brane was shown to determine not only the topology of our brane-leading to a de Sitter, anti-de Sitter, or Minkowski four-dimensional background-but also the properties of the coupling function between the bulk scalar field and the five-dimensional Ricci scalar curvature. In [69], we focused on the case of a positive cosmological constant on the brane, and showed that, in order for the scalar field to be real valued, the coupling function had to be negative over a particular regime in the bulk. Nevertheless, we were able to derive solutions which had a robust four-dimensional effective theory on the brane and a number of interesting, yet provocative, features in the bulk. In a follow-up work [73], we considered the case of a negative cosmological constant on the brane, which allowed positive-definite coupling functions; by employing two particular forms of the latter, we produced two complete analytical solutions that were characterized by a regular scalar field and a localized close-to-our brane energy-momentum tensor. In addition, the solutions featured a negative-definite bulk potential which supported by itself the warping of the space-time even in the absence of the traditional, negative, bulk cosmological constant.

Having covered the cases of a de Sitter and anti-de Sitter space-time on our brane in $[69,73]$, in this third installment we turn our attention to the case of a Minkowski brane, i.e., with a vanishing effective cosmological constant. The objective would be the same, namely to perform a comprehensive study of the complete set of field equations and derive analytical solutions for the gravitational background and scalar field in the bulk. As we will demonstrate, this case is the least restrictive and most flexible of the three, and allows for a variety of profiles for the coupling function and scalar field along the extra coordinate. In order to construct physically acceptable solutions, we will demand the finiteness of both the coupling function and scalar field everywhere in the bulk; in fact, we will consider forms of the coupling function that become trivial at large distances from our brane thus leading to a minimally coupled scalartensor theory in that limit. Even under the above assumptions, we will present a large number of solutions; they will all be characterized by a regular scalar field and a finite energy-momentum tensor localized near our brane. In addition, the bulk potential of the scalar field may take a variety of forms at our will, while supporting in all cases an exponentially decaying warp factor even in the absence of a negative bulk cosmological constant. Negative values of the coupling function in the bulk will not be necessary in our analysis, nevertheless, they will be allowed. The form of the effective theory on the brane will thus be of primary importance and a necessary ingredient of our analysis in the study of each solution presented. We will naturally demand a positive effective gravitational constant on our brane, and investigate whether this demand may be simultaneously satisfied with the condition of a positive total energy of our brane and the validity of the weak energy conditions in the bulk. The gravitational background on the brane will be described by the Schwarzschild solution leading to either a nonhomogeneous black-string solution in the bulk, when the mass parameter $M$ is nonzero, or a regular anti-de Sitter space-time, when $M=0$.

Our paper has the following outline: in Sec. II, we present our theory, the field equations, and impose a number of physical constraints on the scalar field and its coupling function. In Sec. III to VIII, we present a large number of complete braneworld solutions, and discuss in detail their physical properties in the bulk, the junction conditions, the effective theory on the brane and the parameter space where the optimum solutions-from the physical point of view-emerge in each case. We present our conclusions in Sec. IX.

\section{THE THEORETICAL FRAMEWORK}

We consider the following action functional which describes a five-dimensional scalar-tensor theory of gravity

$$
\begin{aligned}
S_{B}= & \int d^{4} x \int d y \sqrt{-g^{(5)}} \\
& \times\left[\frac{f(\Phi)}{2 \kappa_{5}^{2}} R-\Lambda_{5}-\frac{1}{2} \partial_{L} \Phi \partial^{L} \Phi-V_{B}(\Phi)\right] .
\end{aligned}
$$

The theory contains the five-dimensional scalar curvature $R$, a bulk cosmological constant $\Lambda_{5}$, and a five-dimensional scalar field $\Phi$. The latter is characterized by a selfinteracting potential $V_{B}(\Phi)$ and a nonminimal coupling to $R$ via a coupling function $f(\Phi)$. As in our previous works $[69,73]$, we will initially keep this function arbitrary so that our formalism is applicable to a large class of theories. In the above, $\kappa_{5}^{2}=8 \pi G_{5}$, where $G_{5}$ is the fivedimensional gravitational constant $G_{5}$, and $g_{M N}^{(5)}$ is the metric tensor of the five-dimensional space-time. 
Embedded in this five-dimensional space-time is a 3-brane, our four-dimensional world, located at $y=0$ along the extra spatial dimension. The energy content of our brane is described by the following action:

$$
\begin{aligned}
S_{b r} & =\int d^{4} x \sqrt{-g^{(b r)}}\left(\mathcal{L}_{b r}-\sigma\right) \\
& =-\int d^{4} x \int d y \sqrt{-g^{(b r)}}\left[V_{b}(\Phi)+\sigma\right] \delta(y),
\end{aligned}
$$

which should be added to the bulk action (2.1) to complete the theory. The brane Lagrangian $\mathcal{L}_{b r}$ is assumed, for simplicity, to contain only an interaction term $V_{b}(\Phi)$ of the bulk scalar field with the brane, while $\sigma$ is the constant brane self-energy. Also, $g_{\mu \nu}^{(b r)}=g_{\mu \nu}^{(5)}\left(x^{\lambda}, y=0\right)$ is the induced-on-the-brane metric tensor. In what follows, we will denote five-dimensional indices with capital Latin letters $M, N, L, \ldots$ and four-dimensional indices with lower-case Greek letters $\mu, \nu, \lambda, \ldots$ as usual.

The field equations of the theory follow if we vary the complete action $S=S_{B}+S_{b r}$ with respect to the metric tensor $g_{M N}^{(5)}$ and scalar field $\Phi$. Then, we obtain the gravitational field equations

$$
\begin{aligned}
f(\Phi) G_{M N} \sqrt{-g^{(5)}} & =\kappa_{5}^{2}\left[\left(T_{M N}^{(\Phi)}-g_{M N} \Lambda_{5}\right) \sqrt{-g^{(5)}}\right. \\
& \left.-\left[V_{b}(\Phi)+\sigma\right] g_{\mu \nu}^{(b r)} \delta_{M}^{\mu} \delta_{N}^{\nu} \delta(y) \sqrt{-g^{(b r)}}\right],
\end{aligned}
$$

with the energy-momentum tensor of the theory given by the expression

$$
\begin{aligned}
T_{M N}^{(\Phi)}= & \partial_{M} \Phi \partial_{N} \Phi+g_{M N}\left[-\frac{\partial_{L} \Phi \partial^{L} \Phi}{2}-V_{B}(\Phi)\right] \\
& +\frac{1}{\kappa_{5}^{2}}\left[\nabla_{M} \nabla_{N} f(\Phi)-g_{M N} \square f(\Phi)\right],
\end{aligned}
$$

and the scalar-field equation

$$
\begin{aligned}
- & \frac{1}{\sqrt{-g^{(5)}}} \partial_{M}\left(\sqrt{-g^{(5)}} g^{M N} \partial_{N} \Phi\right) \\
= & \frac{\partial_{\Phi} f}{2 \kappa_{5}^{2}} R-\partial_{\Phi} V_{B}-\frac{\sqrt{-g^{(b r)}}}{\sqrt{-g^{(5)}}} \partial_{\Phi} V_{b} \delta(y),
\end{aligned}
$$

respectively.

The form of the five-dimensional gravitational background needs to be specified next. As in $[69,73]$, we consider the following line element:

$$
\begin{aligned}
d s^{2}= & e^{2 A(y)}\left\{-\left[1-\frac{2 m(r)}{r}\right] d v^{2}+2 d v d r\right. \\
& \left.+r^{2}\left(d \theta^{2}+\sin ^{2} \theta d \varphi^{2}\right)\right\}+d y^{2}
\end{aligned}
$$

which describes a five-dimensional space-time warped along the fifth dimension due to the presence of the warp factor $e^{2 A(y)}$. Its four-dimensional part has the form of a generalized Vaidya line element: if $m(r)$ is a constant $M$, this reduces, after a coordinate transformation, to the Schwarzschild solution. The four-dimensional observer at $y=0$ would then see a black-hole line element on the brane; however, its embedding in the extra dimension as in Eq. (2.6) results in the context of the original RandallSundrum model [11,12], in a black-string solution [14] with an infinitely long singularity plagued by instabilities $[54,55]$.

By introducing a dependence of the mass function on the extra coordinate $y$, it is possible to localize the black hole close to the brane but this demands a form of bulk matter that cannot be supported by ordinary fields $[18,22]$. A more general ansatz for the mass function of the form $m=m(v, r, y)$, that was employed in subsequent works $[31,38]$, increased the flexibility of the model but failed, too, to lead to localized black-hole solutions in the context of a variety of scalar-field theories. Up to today, the analytical determination-in a closed form-of regular, localized black holes in warped braneworld models remains an open problem.

However, the five-dimensional scalar-tensor theory of gravity described by Eq. (2.1) was shown $[31,38]$ to admit novel black-string solutions that may be constructed analytically. In our previous works $[69,73]$, we performed a comprehensive study of the types of black-string solutions that emerge in the context of this theory when the cosmological constant on the brane is positive or negative, respectively. Here, we complete our study by considering the case of a Minkowski brane. As we will demonstrate, this case is the most flexible of all that allows for a larger variety of profiles for the scalar field and its coupling function while retaining all the attractive characteristics of the previous two cases.

We will employ again the line element (2.6), and proceed to derive the explicit form of the field equations (2.3)-(2.5). We will focus on solving this set of equations first in the bulk, and thus ignore for now all $\delta(y)$ terms. The explicit form of the gravitational equations follows by combining the nonvanishing components of the Einstein $G^{M}{ }_{N}$ and energy-momentum $T^{(\Phi) M}{ }_{N}$ tensors. In mixed form, these are

$$
\begin{aligned}
& G_{0}^{0}=G^{1}{ }_{1}=6 A^{\prime 2}+3 A^{\prime \prime}-\frac{2 e^{-2 A} \partial_{r} m}{r^{2}}, \\
& G_{2}^{2}=G^{3}{ }_{3}=6 A^{\prime 2}+3 A^{\prime \prime}-\frac{e^{-2 A} \partial_{r}^{2} m}{r} \\
& G_{4}^{4}=6 A^{\prime 2}-\frac{e^{-2 A}\left(2 \partial_{r} m+r \partial_{r}^{2} m\right)}{r^{2}}
\end{aligned}
$$

and 


$$
\begin{aligned}
& T^{(\Phi) 0}{ }_{0}=T^{(\Phi) 1}{ }_{1}=T^{(\Phi) 2}{ }_{2}=T^{(\Phi) 3}{ }_{3}=A^{\prime} \Phi^{\prime} \partial_{\Phi} f+\mathcal{L}_{\Phi}-\square f, \\
& T^{(\Phi) 4}{ }_{4}=\left(1+\partial_{\Phi}^{2} f\right) \Phi^{\prime 2}+\Phi^{\prime \prime} \partial_{\Phi} f+\mathcal{L}_{\Phi}-\square f,
\end{aligned}
$$

respectively, where a prime $\left({ }^{\prime}\right)$ denotes the derivative with respect to the $y$ coordinate. Above, we have made the assumption that the scalar field depends only on the coordinate along the fifth dimension, i.e., $\Phi=\Phi(y)$, and we have defined the quantities

$\mathcal{L}_{\Phi}=-\frac{1}{2} \partial_{L} \Phi \partial^{L} \Phi-V_{B}(\Phi)=-\frac{1}{2} \Phi^{\prime 2}-V_{B}(\Phi)$,

and

$$
\square f=4 A^{\prime} \Phi^{\prime} \partial_{\Phi} f+\Phi^{\prime 2} \partial_{\Phi}^{2} f+\Phi^{\prime \prime} \partial_{\Phi} f .
$$

Employing the above, and upon some simple manipulation [69], we obtain three equations having the following form:

$$
\begin{aligned}
r \partial_{r}^{2} m-2 \partial_{r} m & =0 \\
f\left(3 A^{\prime \prime}+e^{-2 A} \frac{\partial_{r}^{2} m}{r}\right) & =\partial_{\Phi} f\left(A^{\prime} \Phi^{\prime}-\Phi^{\prime \prime}\right)-\left(1+\partial_{\Phi}^{2} f\right) \Phi^{\prime 2}
\end{aligned}
$$

$$
f\left(6 A^{\prime 2}+3 A^{\prime \prime}-\frac{2 e^{-2 A} \partial_{r} m}{r^{2}}\right)=A^{\prime} \Phi^{\prime} \partial_{\Phi} f+\mathcal{L}_{\Phi}-\square f-\Lambda_{5} .
$$

Note that, for notational simplicity, we have absorbed the gravitational constant $\kappa_{5}^{2}$ in the expression of the general coupling function $f(\Phi)$. Turning next to the scalar-field equation in the bulk (2.5), this takes the explicit form

$$
\begin{aligned}
\Phi^{\prime \prime}+4 A^{\prime} \Phi^{\prime}= & \partial_{\Phi} f\left(10 A^{\prime 2}+4 A^{\prime \prime}-e^{-2 A} \frac{2 \partial_{r} m+r \partial_{r}^{2} m}{r^{2}}\right) \\
& +\partial_{\Phi} V_{B} .
\end{aligned}
$$

In order to increase the flexibility of the theory, the form of the mass function $m=m(r)$ in the gravitational background (2.6) was left arbitrary. Nevertheless, this will be duly determined via Eq. (2.11); by direct integration, we obtain the unique solution

$$
m(r)=M+\Lambda r^{3} / 6
$$

where $M$ and $\Lambda$ are arbitrary integration constants. The projected-on-the-brane gravitational background follows by setting $y=0$ in the line element (2.6) and using the above result for the mass function; then, we find the expression

$$
\begin{aligned}
d s_{4}^{2}= & -\left(1-\frac{2 M}{r}-\frac{\Lambda r^{2}}{3}\right) d v^{2}+2 d v d r \\
& +r^{2}\left(d \theta^{2}+\sin ^{2} \theta d \varphi^{2}\right)
\end{aligned}
$$

By employing an appropriate coordinate transformation, the above Vaidya form of the four-dimensional line element may be transformed to the usual Schwarzschild-(anti-)de Sitter solution [69]. As a result, we may interpret the two arbitrary parameters $M$ and $\Lambda$ as the mass of the black hole on the brane and the cosmological constant on the brane. The cases of positive and negative cosmological constant on the brane (i.e., $\Lambda>0$ and $\Lambda<0$ ) were studied respectively in our previous two works $[69,73]$; in the context of the present analysis, we will focus on the case of a zero four-dimensional cosmological constant $(\Lambda=0)$.

Returning to the remaining field equations (2.12)-(2.14), one may demonstrate that only two of them are independent [69]. We may therefore ignore altogether the scalar field equation (2.14) and work only with the gravitational equations (2.12)-(2.13). The former equation will provide the solution for the scalar field $\Phi$ while the latter will help us to determine the scalar potential in the bulk $V_{B}(\Phi)$. To this end, we need also the expression of the warp function $A(y)$ for which we will use the well-known form $A(y)=$ $-k|y|[11,12]$, with $k$ a positive constant, as this ensures the localization of gravity near the brane. Setting also the mass function to be $m(r)=M$ (since $\Lambda=0$ ), Eq. (2.12) takes the form ${ }^{1}$

$$
\left(1+\partial_{\Phi}^{2} f\right) \Phi^{\prime 2}+\partial_{\Phi} f\left(\Phi^{\prime \prime}+k \Phi^{\prime}\right)=0,
$$

or

$$
\Phi^{\prime 2}+\partial_{y}^{2} f+k \partial_{y} f=0
$$

while Eq. (2.13), with the use of Eq. (2.18), can be solved for $V_{B}(y)$ :

$$
V_{B}(y)=-\Lambda_{5}-6 k^{2} f(y)+\frac{7}{2} k \partial_{y} f-\frac{1}{2} \partial_{y}^{2} f
$$

In the above, we have also used the relations

$$
\partial_{y} f=\Phi^{\prime} \partial_{\Phi} f, \quad \partial_{y}^{2} f=\Phi^{\prime 2} \partial_{\Phi}^{2} f+\Phi^{\prime \prime} \partial_{\Phi} f .
$$

The topology of the five-dimensional bulk space-time may be inferred from the form of the curvature invariant quantities. Using the five-dimensional line element (2.6), together with the relations $m(r)=M$ and $A=-k|y|$, we find the following expressions:

\footnotetext{
${ }^{1}$ We assume a $\mathbf{Z}_{2}$ symmetry in the bulk under the change $y \rightarrow$ $-y$ therefore, henceforth, we focus on the positive $y$ regime.
} 


$$
\begin{aligned}
R & =-20 k^{2}, \quad R_{M N} R^{M N}=80 k^{4}, \\
R_{M N R S} R^{M N R S} & =40 k^{4}+\frac{48 M^{2} e^{4 k|y|}}{r^{6}} .
\end{aligned}
$$

For $M=0$, the bulk space-time is characterized by a constant negative curvature at every point, and is therefore an AdS space-time. This holds despite the presence of a nontrivial distribution of energy in the bulk, i.e., that of a nonminimally coupled scalar field with a potential, and is ensured through the field equations which, like Eqs. (2.18) and (2.19), relate the different bulk quantities among themselves. It is for this reason that, as we will see, the exponentially decaying warp factor will be supported even in the absence of the negative bulk cosmological constant $\Lambda_{5}$. In the case where $M \neq 0$, the above invariants describe a five-dimensional black-string solution with an infinitely long space-time singularity extending throughout the extra dimension. The black-string singularity reaches the boundary of space-time which is by itself a singular hypersurface.

The solution for both the scalar field and the bulk potential depends, through Eqs. (2.18)-(2.19), on the form of the nonminimal coupling function $f(\Phi)$. In our previous work [73], we assigned the following constraints to the scalar field $\Phi(y)$ and its coupling function $f[\Phi(y)]$ :

(i) Both functions should be real and finite in their whole domain and of class $C^{\infty}$.

(ii) At $y \rightarrow \pm \infty$, both functions should satisfy the following relations, otherwise the finiteness of the theory at infinity cannot be ascertained:

$$
\begin{array}{ll}
\lim _{y \rightarrow \pm \infty} \frac{d^{n}[f(y)]}{d y^{n}}=0, & \forall n \geq 1, \\
\lim _{y \rightarrow \pm \infty} \frac{d^{n}[\Phi(y)]}{d y^{n}}=0, & \forall n \geq 1 .
\end{array}
$$

The second constraint amounts to considering profiles of the scalar field and forms of the coupling function that both reduce to a constant value far away from the brane. Together with the first constraint, they ensure a physically acceptable behavior for our scalar-tensor theory. The sign, however, of the coupling function $f(y)$ will not be fixed. In [69], where the case of a positive cosmological constant on the brane was studied, i.e., $\Lambda>0$, the coupling function had to be negative-definite away from our brane for the reality of the scalar field to be ensured; nevertheless, the effective theory on the brane could still be well defined. In the case of $\Lambda<0$ [73], no such requirement was necessary and the coupling function was assumed to be everywhere positive-definite in terms of the $y$ coordinate; then, gravity was normal over the entire five-dimensional space-time leading to a well-defined effective field theory on the brane.

In the context of the present analysis, where $\Lambda=0$, we may consider coupling functions that are either positive- or negative-definite for particular regions of the $y$ coordinate. As we will demonstrate, it is possible to obtain a positive effective four-dimensional gravitational constant in every case. This will hold even when five-dimensional gravity behaves in an antigravitating way at particular regimes of space-time-as it turns out, such a behavior is not physically forbidden as long as the effective theory on our brane is well defined. To this end, the derivation of the effective theory on the brane is going to play an important role in our forthcoming analysis, and will thus supplement every bulk solution we derive.

\section{A LINEAR COUPLING FUNCTION}

Choosing $\Lambda=0$ on our brane simplifies the set of field equations of the theory, but more importantly, relaxes constraints that had to be imposed on the coupling function. As a result, the latter is now allowed to adopt a variety of physically acceptable forms, all obeying the criteria (i) and (ii) of the previous section. These forms lead to viable braneworld models (for $M=0$ ) or black-string solutions (for $M \neq 0$ ). In an effort to construct the most realistic solutions, we will also study, in every case, the energy conditions both in the bulk and on the brane.

We start our analysis with the case of the linear coupling function:

$$
f(\Phi)=f_{0}+\Phi_{0} \Phi
$$

where $f_{0}$ and $\Phi_{0}$ are arbitrary parameters of the theory. In what follows, we will first solve the system of field equations (2.17) and (2.19) in the bulk and then consider the effective theory on the brane as well as the energy conditions.

\section{A. The bulk solution}

Substituting the aforementioned coupling function in Eq. (2.17) and solving the resulting second-order differential equation, we obtain the solution:

$$
\Phi(y)=\Phi_{0}\left[-k y+\ln \left(e^{k y}+\xi\right)\right]
$$

where $\xi$ is an integration constant. Note that the gravitational field equation (2.17) possesses a translational symmetry with respect to the scalar field $\Phi(y)$. Hence, we are free to fix the value of a second integration constant, that should in principle appear additively on the right-hand side of Eq. (3.2), to zero without loss of generality. Then, using Eq. (3.2) in (3.1), we find

$$
f(y)=f_{0}+\Phi_{0}^{2}\left[-k y+\ln \left(e^{k y}+\xi\right)\right] .
$$

As we mentioned earlier, both functions $f(y)$ and $\Phi(y)$ should be real and finite; therefore $\xi \in(-1,0) \cup(0, \infty)$, and $\Phi_{0} \in \mathbb{R} \backslash\{0\}$. It is clear from Eqs. (3.2) and (3.3) that if 


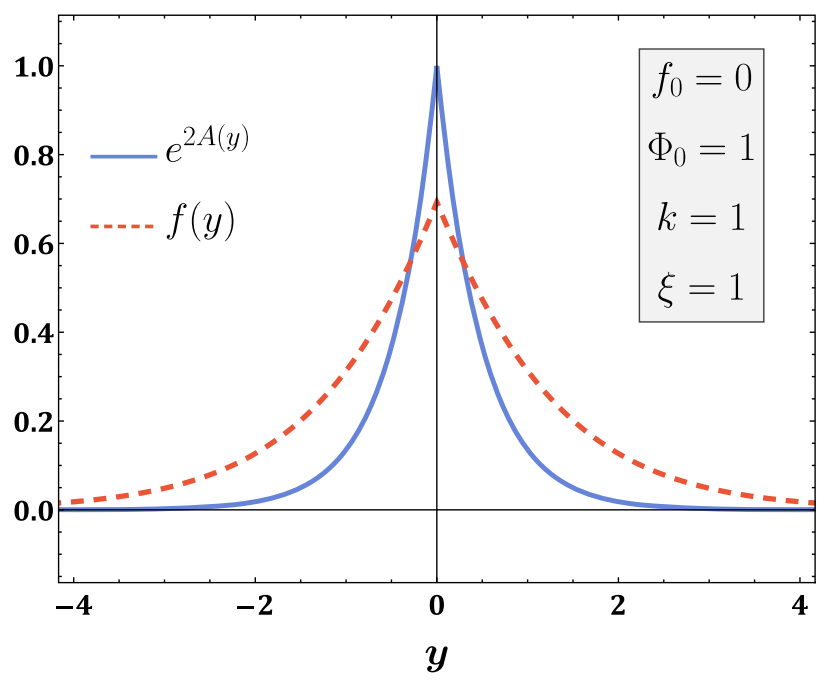

(a)

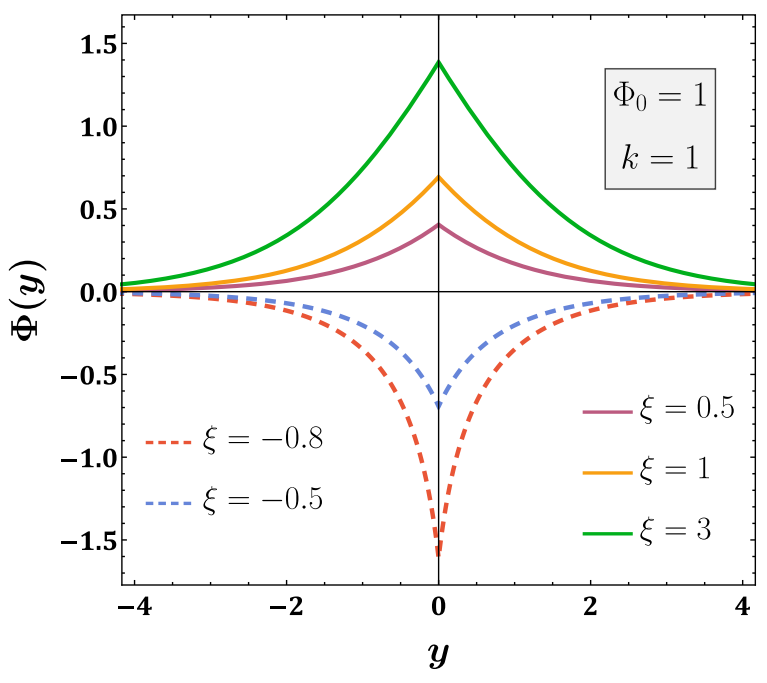

(b)

FIG. 1. (a) The warp factor $e^{2 A(y)}=e^{-2 k|y|}$ and coupling function $f(y)$ in terms of the coordinate $y$ for $f_{0}=0, \Phi_{0}=1, k=1, \xi=1$, and (b) the scalar field $\Phi(y)$ for different values of the parameter $\xi=-0.8,-0.5,0.5,1,3$ (from bottom to top).

we allow $\xi$ to become equal to zero, then we nullify the scalar field everywhere in the bulk and reduce the coupling function to a constant, which makes our model trivial. The allowed range of values for the parameter $f_{0}$ will be determined shortly.

In Fig. 1(a), we depict the warp factor $e^{2 A(y)}=e^{-2 k|y|}$ and coupling function $f(y)$ in terms of the coordinate $y$ for $f_{0}=0, \Phi_{0}=1, k=1$, and $\xi=1$. We observe that, similarly to the warp factor, the coupling function remains localized close to our brane and reduces to zero at large distances although with a smaller rate. According to this behavior, the nonminimal coupling of the scalar field to the five-dimensional Ricci scalar takes its maximum value at the location of the brane whereas, for large values of $y$, this coupling vanishes leading to a minimally coupled scalartensor theory of gravity. The profile of the scalar field $\Phi(y)$ itself is presented in Fig. 1(b) for $\Phi_{0}=1$ and $k=1$. We also display the dependence of this profile on the value of the parameter $\xi=-0.8,-0.5,0.5,1,3$ (from bottom to top). It is clear that also the scalar field exhibits a localized behavior with the value of $\xi$ determining the overall sign and maximum value of $\Phi$ on our brane. The dependence of the coupling function $f(y)$ on the value of $\xi$ is similar to that of the scalar field, as one can easily deduce from the relation (3.1).

The potential of the scalar field $V_{B}(y)$ in the bulk can be determined from Eq. (2.19) using the expression of the coupling function $f(y)$ (3.3). Thus, we obtain

$$
\begin{aligned}
V_{B}(y)= & -\Lambda_{5}-6 k^{2} f_{0}+\frac{k^{2} \Phi_{0}^{2}}{2}\left[12 k y-\frac{\xi\left(8 e^{k y}+7 \xi\right)}{\left(\xi+e^{k y}\right)^{2}}\right] \\
& -6 k^{2} \Phi_{0}^{2} \ln \left(\xi+e^{k y}\right) .
\end{aligned}
$$

Using Eq. (3.2), we can express the potential in terms of the scalar field in a closed form, as follows:

$$
\begin{aligned}
V_{B}(\Phi)= & -\Lambda_{5}-6 k^{2} f_{0}-6 k^{2} \Phi_{0} \Phi-4 k^{2} \Phi_{0}^{2}\left(1-e^{-\Phi / \Phi_{0}}\right) \\
& +\frac{k^{2} \Phi_{0}^{2}}{2}\left(1-e^{-\Phi / \Phi_{0}}\right)^{2} .
\end{aligned}
$$

We observe that the parameter $f_{0}$ appearing in the expression of the coupling function (3.3) gives a constant contribution to the scalar bulk potential. Depending on the value of $f_{0}$, the asymptotic value of $V_{B}$ in the bulk (when $\Phi$ vanishes) can be either positive, zero, or negative. In the latter case, this contribution may be considered to play the role of the negative bulk cosmological constant $\Lambda_{5}$, which is usually introduced in an $a d$ hoc way. Therefore, such a quantity is not necessary any more in order to support the exponentially decreasing warp factor á la Randall Sundrum $[11,12]$. As mentioned earlier, it is the nonminimal coupling of the scalar field combined with the form of the bulk potential that supports the AdS bulk space-time and the chosen form of the warp factor. To this end, we will henceforth choose a vanishing value for $\Lambda_{5}$ in any numerical evaluation; however, for completeness, we will retain it in our equations. The profile of the bulk potential $V_{B}$ is presented in Fig. 2(a) for $f_{0}=1$, which leads to a negative asymptotic value of $V_{B}$. The figure depicts the dependence of $V_{B}$ on the parameter $\xi$ : the scalar potential may be negative everywhere in the bulk or assume a positive value on our brane depending on the value of $\xi$.

We may also compute the components of the energymomentum tensor of the theory in the bulk. Using the relations $\rho=-T_{0}^{0}, p^{i}=T^{i}{ }_{i}, p^{y}=T^{y}{ }_{y}$, we obtain the following expressions: 


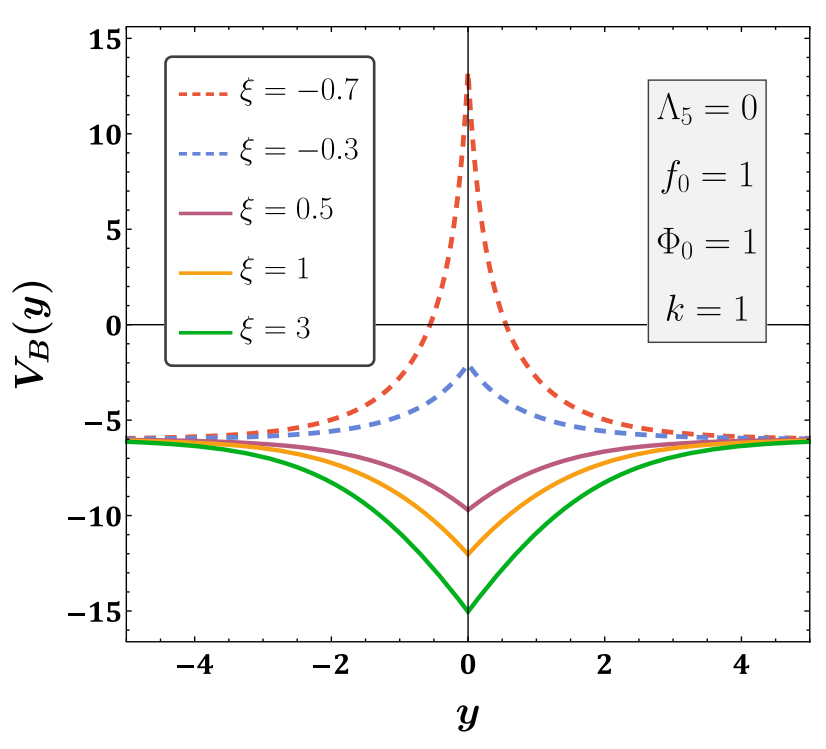

(a)

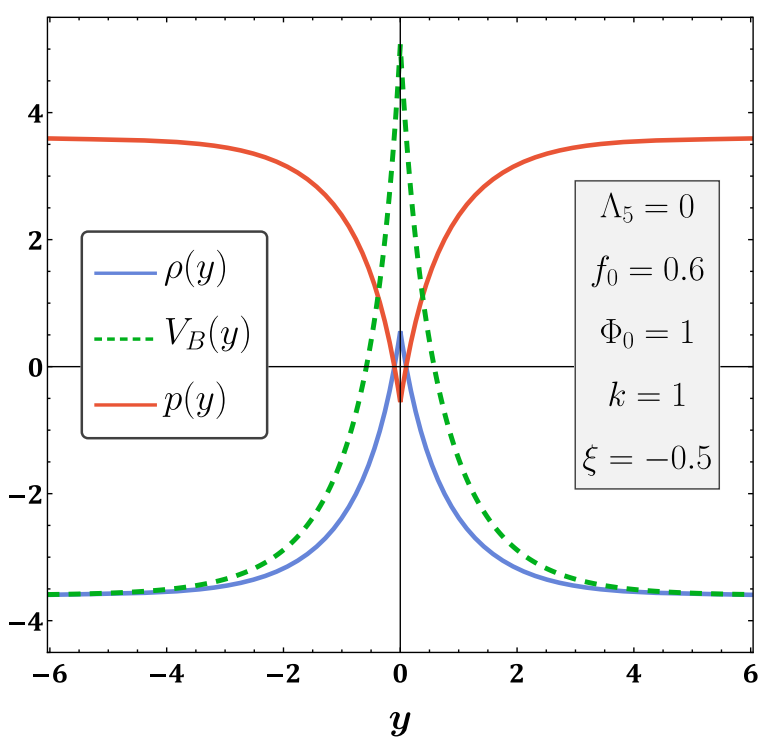

(b)

FIG. 2. (a) Scalar potential $V_{B}$ in terms of the coordinate $y$ for different values of the parameter $\xi=-0.7,-0.3,0.5,1,3$ (from top to bottom). (b) Energy density $\rho$, pressure components $p^{i}=p^{y}=p$, and scalar potential $V_{B}$ in terms of the coordinate $y$ for the case $f_{0}=0.6$ and $\xi=-0.5$.

$$
\begin{aligned}
& \rho(y)=-\left(T_{0}^{(\Phi) 0}-\Lambda_{5}\right)=-6 k^{2} f(y), \\
& p^{i}(y)=T_{i}^{(\Phi) i}-\Lambda_{5}=6 k^{2} f(y) \\
& p^{y}(y)=T_{y}^{(\Phi) y}-\Lambda_{5}=6 k^{2} f(y)
\end{aligned}
$$

The above relations hold in general, for an arbitrary form of the coupling function and profile of the scalar field. From the above expressions, we can immediately observe that the energy-momentum tensor in the bulk is isotropic $\left(p^{y}=p^{i} \equiv p\right)$ and satisfies an equation of state of the form $p=-\rho$. The sign of all energy-momentum tensor components depends on that of the coupling function: at bulk regimes where $f(y)$ is negative-definite, the energy density $\rho(y)$ will be positive while the pressure $p(y)$ would have the opposite sign. At these regimes, the weak energy conditions ${ }^{2}$ will be satisfied. We are primarily interested in satisfying these on and close to our brane. Thus, if we impose the condition that $f(0)<0$ and combine this inequality with the form of Eq. (3.3), we may obtain the range of values for the parameter $f_{0}$, with respect to $\xi$ and $\Phi_{0}$, for which the weak energy conditions on our brane are satisfied. Hence, we get

$$
\frac{f_{0}}{\Phi_{0}^{2}}<-\ln (1+\xi)
$$

A particular indicative case where the weak energy conditions are satisfied on our brane is depicted in

\footnotetext{
${ }^{2}$ The weak energy conditions postulate that $\rho \geq 0, \rho+p \geq 0$.
}

Fig. 2(b): it corresponds to the set of values $\Phi_{0}=1$, $\xi=-0.5$, and $f_{0}=0.6$, which satisfy the above inequality. Both the bulk potential and energy density are positive on our brane while the pressure components assume a negative value of equal magnitude to that of $\rho$.

\section{B. Junction conditions and effective theory}

Let us now address the junction conditions that should be imposed on our bulk solution due to the presence of the brane at $y=0$. The energy content of the brane will be given by the combination $\sigma+V_{b}(\Phi)$, where $\sigma$ is the constant self-energy of the brane and $V_{b}(\Phi)$ an interaction term of the bulk scalar field with the brane. Since this distribution of energy is located at a single point along the extra dimension, i.e., at $y=0$, it creates a discontinuity in the second derivatives of the warp factor, the coupling function, and the scalar field at the location of the brane. We may then write $A^{\prime \prime}=\hat{A}^{\prime \prime}+\left[A^{\prime}\right] \delta(y), f^{\prime \prime}=\hat{f}^{\prime \prime}+\left[f^{\prime}\right] \delta(y)$, and $\Phi^{\prime \prime}=\hat{\Phi}^{\prime \prime}+\left[\Phi^{\prime}\right] \delta(y)$, where the hat quantities denote the distributional (i.e., regular) parts of the second derivatives and $[\cdots]$ stand for the discontinuities of the corresponding first derivatives across the brane [81]. Then, in the complete field equations (2.12) and (2.14), we match the coefficients of the $\delta$-function terms ${ }^{3}$ and obtain the following two conditions:

$$
3 f(y)\left[A^{\prime}\right]=-\left[\Phi^{\prime}\right] \partial_{\Phi} f-\left(\sigma+V_{b}\right),
$$

\footnotetext{
${ }^{3}$ We note that the line element (2.6) satisfies the relation $\sqrt{-g^{(5)}}=\sqrt{-g^{(4)}}$.
} 


$$
\left[\Phi^{\prime}\right]=4\left[A^{\prime}\right] \partial_{\Phi} f+\partial_{\Phi} V_{b}
$$

respectively, where all quantities are evaluated at $y=0$. The above expressions also hold in general for arbitrary forms of the coupling function $f(\Phi)$. In the case of a linear $f(\Phi)$, employing the form of the warp function $A(y)=$ $-k|y|$ and the solution (3.2) for the scalar field $\Phi(y)$, we obtain the constraints

$$
\begin{aligned}
\sigma+\left.V_{b}(\Phi)\right|_{y=0} & =\frac{2 k \xi \Phi_{0}^{2}}{1+\xi}+6 k f_{0}+6 k \Phi_{0}^{2} \ln (1+\xi), \\
\left.\partial_{\Phi} V_{b}\right|_{y=0} & =\frac{2 k \Phi_{0}(4+3 \xi)}{1+\xi} .
\end{aligned}
$$

In the above relations, we have used the assumed $\mathbf{Z}_{2}$ symmetry in the bulk.

The first constraint (3.12) relates the total energy density of the brane with bulk parameters. It may be used to fix one of the bulk parameters of our solution, for example, the warping constant $k$; then, the warping of space-time is naturally determined by the distribution of energy in the bulk and on the brane. The second constraint (3.13) may in turn be used to fix one parameter of the brane interaction term $V_{b}$ of the scalar field. Going further, we may demand that, for physically interesting situations, the total energy density of the brane should be positive; then, the right-hand side of Eq. (3.12) leads to

$$
\frac{f_{0}}{\Phi_{0}^{2}}>-\ln (1+\xi)-\frac{\xi}{3(1+\xi)} .
$$

The above is therefore an additional constraint that the bulk parameters $\left(f_{0}, \Phi_{0}, \xi\right)$ should satisfy which, as the one of Eq. (3.9), follows not from the mathematical consistency of the solution but from strictly physical arguments.

We now turn to the effective theory on the brane that follows by integrating the complete five-dimensional theory, given by $S=S_{B}+S_{b r}$, over the fifth coordinate $y$. We would like to derive first the effective four-dimensional gravitational constant that governs all gravitational interactions on our brane. For this, it is of key importance to express the five-dimensional Ricci scalar $R$ in terms of the four-dimensional projected-on-the-brane Ricci scalar $R^{(4)}$. One can easily prove that the five-dimensional Ricci scalar $R$ of the following line element

$$
d s^{2}=e^{-2 k|y|} g_{\mu \nu}^{(b r)}(x) d x^{\mu} d x^{\nu}+d y^{2}
$$

can be written in the form

$$
R=-20 k^{2}+8 k \frac{d^{2}|y|}{d y^{2}}+e^{2 k|y|} R^{(4)} .
$$

Equation (3.16) holds even if the projected-on-thebrane four-dimensional metric $g_{\mu \nu}^{(b r)}$ leads to a zero four-dimensional Ricci scalar $R^{(4)}$ when the latter is evaluated for particular solutions (as is the case for our Vaidya induced metric). The part of the complete action $S=S_{B}+S_{b r}$ that is relevant for the evaluation of the effective gravitational constant is the following:

$$
S \supset \int d^{4} x d y \sqrt{-g^{(5)}} \frac{f(\Phi)}{2} e^{2 k|y|} R^{(4)}
$$

Then, using also that $\sqrt{-g^{(5)}}=e^{-4 k|y|} \sqrt{-g^{(b r)}}$, where $g_{\mu \nu}^{(b r)}$ is the metric tensor of the projected-on-the-brane space-time, the four-dimensional, effective gravitational constant is given by the integral

$$
\begin{aligned}
\frac{1}{\kappa_{4}^{2}} & \equiv 2 \int_{0}^{\infty} d y e^{-2 k y} f(y) \\
& =2 \int_{0}^{\infty} d y e^{-2 k y}\left[f_{0}-\Phi_{0}^{2} k y+\Phi_{0}^{2} \ln \left(e^{k y}+\xi\right)\right] .
\end{aligned}
$$

Using the relation $1 / \kappa_{4}^{2}=M_{P l}^{2} / 8 \pi$ and calculating the above integral, we obtain the following expression for the effective Planck scale:

$M_{\mathrm{Pl}}^{2}=\frac{8 \pi \Phi_{0}^{2}}{k}\left\{\frac{f_{0}}{\Phi_{0}^{2}}-\frac{1}{2}+\frac{1}{\xi^{2}}\left[\xi+\left(\xi^{2}-1\right) \ln (1+\xi)\right]\right\}$.

Note, that, due to the localization of both the coupling function and scalar field close to our brane, no need arises for the introduction of a second brane in the model. The above value for $M_{\mathrm{Pl}}^{2}$ is therefore finite as demanded, however, it is not sign-definite. We should therefore demand that the aforementioned expression is positivedefinite which leads to the third, and most, important constraint on the values of $\left(f_{0}, \Phi_{0}, \xi\right)$, namely

$$
\frac{f_{0}}{\Phi_{0}^{2}}>\frac{\xi-2}{2 \xi}+\frac{1-\xi^{2}}{\xi^{2}} \ln (1+\xi)
$$

The integral of all the remaining terms of the fivedimensional action $S=S_{B}+S_{b r}$, apart from the one appearing in Eq. (3.17), will yield the effective cosmological constant on the brane. This is due to the fact that the scalar field $\Phi$ is only $y$ dependent; therefore, when the integration over the extra coordinate $y$ is performed, no dynamical degree of freedom remains in the four-dimensional effective theory. The effective cosmological constant is thus given by the expression 


$$
\begin{aligned}
-\Lambda_{4}= & \int_{-\infty}^{\infty} d y e^{-4 k|y|}\left[-10 k^{2} f(y)-\Lambda_{5}-\frac{1}{2} \Phi^{\prime 2}-V_{B}(y)\right. \\
& \left.+\left.f(y)\left(-4 A^{\prime \prime}\right)\right|_{y=0}-\left[\sigma+V_{b}(\Phi)\right] \delta(y)\right] \\
= & 2 \int_{0}^{\infty} d y e^{-4 k y}\left[-10 k^{2} f(y)-\Lambda_{5}-\frac{1}{2} \Phi^{\prime 2}-V_{B}(y)\right] \\
& +8 k f(0)-\left[\sigma+V_{b}(\Phi)\right]_{y=0} .
\end{aligned}
$$

In the above, we have also added the Gibbons-Hawking term [82] due to the presence of the brane, that acts as a boundary for the five-dimensional space-time. Substituting the expressions for the coupling function and the bulk potential of the scalar field, and employing the junction condition (3.12), we finally obtain the result

$$
\Lambda_{4}=0 \text {. }
$$

As in our previous works for positive [69] and negative cosmological constant [73] on the brane, it is clear that the parameter $\Lambda$ appearing in the expression of the mass function (2.15), and in the projected-on-the-brane line element (2.16) is indeed related to the four-dimensional cosmological constant $\Lambda_{4}$. Therefore, in the context of the present analysis where we have set $\Lambda=0$, we derived a vanishing $\Lambda_{4}$ as anticipated.

\section{The energy conditions in the parameter space}

We will now focus on the inequalities (3.9), (3.14), and (3.20), and in particular investigate whether it is possible to simultaneously satisfy all three of them. To this end, we study the parameter space defined by the ratio $f_{0} / \Phi_{0}^{2}$ and the parameter $\xi$ : this is depicted in Fig. 3, where we have plotted the expressions of the right-hand sides of the inequalities (3.9), (3.14), (3.20) with respect to the parameter $\xi$. From a physical point of view, the most important inequality to satisfy is (3.20), which ensures that the fourdimensional effective gravitational constant on our brane is positive: this demands that $f_{0} / \Phi_{0}^{2}$ should be always greater than $\frac{\xi-2}{2 \xi}+\frac{1-\xi^{2}}{\xi^{2}} \ln (1+\xi)$ and corresponds to the area above the red dashed curve in Fig. 3. The inequality (3.9) ensures that the bulk energy-momentum tensor satisfies the weak energy conditions at the location of our brane, and demands that $f_{0} / \Phi_{0}^{2}$ should be smaller than $-\ln (1+\xi)$; this corresponds to the area below the purple continuous line in Fig. 3. Finally, inequality (3.14) expresses the demand that the total energy density of our brane is positive; this is satisfied if $f_{0} / \Phi_{0}^{2}$ is greater than $-\ln (1+\xi)-\frac{\xi}{3(1+\xi)}$; this is the area above the blue dashed curve in Fig. 3.

It is straightforward to see that it is impossible to satisfy all three inequalities simultaneously. However, it is always possible to satisfy two out of these three at a time-in Fig. 3, we have highlighted the regions where the most important inequality (3.20) is one of the two satisfied

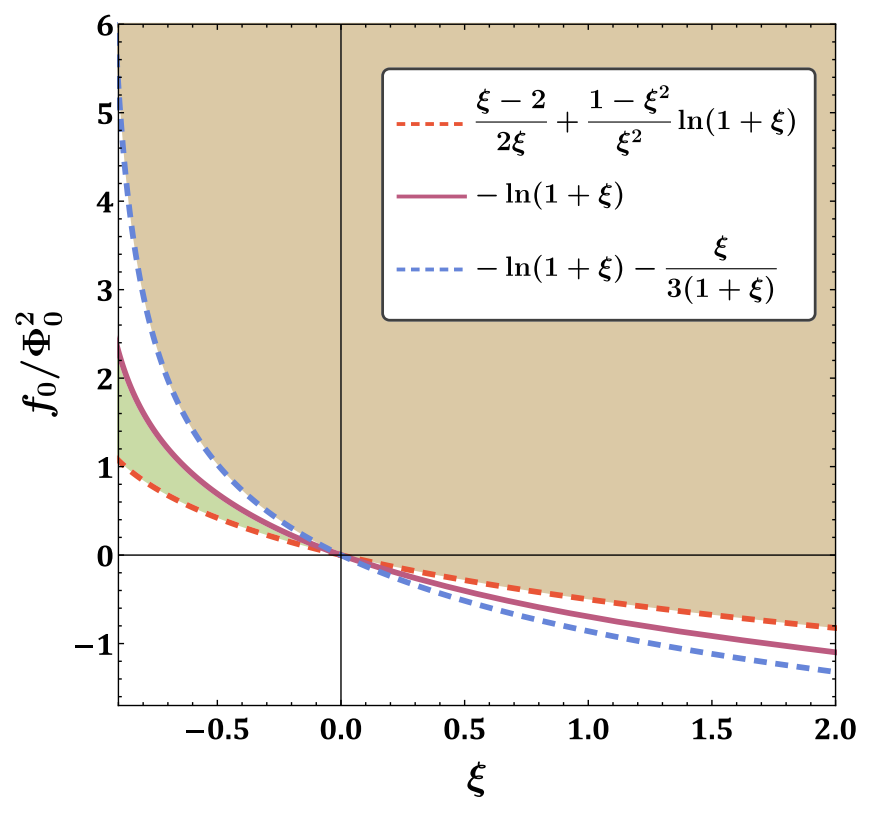

FIG. 3. The parameter space between the ratio $f_{0} / \Phi_{0}^{2}$ and the parameter $\xi$. The graphs of the expressions of the right-hand side of the inequalities (3.9), (3.14), (3.20) are depicted as well.

conditions-we observe that this area covers a very large part of the parameter space. Which one of the two remaining inequalities is the second satisfied condition depends on the value of the parameter $\xi$; therefore, we distinguish the following cases:

(i) For $\xi \in(-1,0)$, it is easy to see that the following sequence of inequalities holds:

$$
\begin{aligned}
& \frac{\xi-2}{2 \xi}+\frac{1-\xi^{2}}{\xi^{2}} \ln (1+\xi)<-\ln (1+\xi)<-\ln (1+\xi) \\
& -\frac{\xi}{3(1+\xi)}
\end{aligned}
$$

Thus, we can simultaneously satisfy either the inequalities (3.20) and (3.9) (green region in Fig. 3) or (3.20) and (3.14) (brown region in Fig. 3). In the former case, we have a physically acceptable fourdimensional effective theory on the brane and the weak energy conditions are satisfied on and close to our brane; the total energy density of the brane $\sigma+\left.V_{b}(\Phi)\right|_{y=0}$, however, is negative. In the latter case, we still have a physically acceptable effective theory and the total energy density of our brane is now positive; the weak energy conditions though are not satisfied by the bulk matter close to our brane.

(ii) For $\xi>0$, it now holds

$$
\begin{gathered}
-\ln (1+\xi)-\frac{\xi}{3(1+\xi)}<-\ln (1+\xi) \\
<\frac{\xi-2}{2 \xi}+\frac{1-\xi^{2}}{\xi^{2}} \ln (1+\xi) .
\end{gathered}
$$


In this case, we are able to simultaneously satisfy only the inequalities (3.20) and (3.14) (brown region in Fig. 3). Then, we can have a regular fourdimensional effective theory and a positive total energy density on our brane. However, in this range of values for the parameter $\xi$, it is impossible to satisfy the weak energy condition close to our brane and have a well-behaved effective theory.

Going back to Figs. 2(a) and 2(b), we observe that the solution depicted in Fig. 2(b) as well as the solution for $\xi=-0.7$ in Fig. 2(a) fall in the green area of Fig. 3 and thus respect the energy conditions-indeed $V_{B}$ and $\rho$ are positive on and close to the brane. In contrast, the remaining solutions of Fig. 2(a) belong to the brown area of Fig. 3, and thus violate the weak energy conditions; they have, however, a positive total energy density through the junction condition (3.12). We stress that all depicted solutions have a well-defined four-dimensional effective theory, i.e., a positive effective gravitational constant.

\section{A QUADRATIC COUPLING FUNCTION}

In this section, we proceed to consider the case of the quadratic coupling function, and thus we write

$$
f(\Phi)=f_{0}+\Phi_{0} \Phi+\lambda \Phi^{2},
$$

where again $\left(f_{0}, \Phi_{0}, \lambda\right)$ are arbitrary parameters. Throughout this section, it will be assumed that $\lambda \neq 0$ otherwise the analysis reduces to the one of the linear case studied in the previous section. As before, we start with the derivation of the bulk solution and then turn to the effective theory on the brane.

\section{A. The bulk solution and the effective theory on the brane}

Substituting the aforementioned form of the coupling function in Eq. (2.17) we obtain the equation:

$$
(1+2 \lambda) \Phi^{\prime 2}+\left(2 \lambda \Phi+\Phi_{0}\right)\left(\Phi^{\prime \prime}+k \Phi^{\prime}\right)=0 .
$$

Integrating, we find the following solution for the scalar field:

$\Phi(y)=\left\{\begin{array}{ll}\frac{1}{2 \lambda}\left[\Phi_{1}\left(\mu+e^{-k y}\right)^{\frac{2 \lambda}{1+4 \lambda}}-\Phi_{0}\right], & \lambda \in \mathbb{R} \backslash\left\{-\frac{1}{4}, 0\right\} \\ 2 \Phi_{0}+\Phi_{1} e^{\mu e^{-k y}}, & \lambda=-\frac{1}{4}\end{array}\right\}$,

where $\mu$ and $\Phi_{1}$ are integration constants. We note that the case with $\Phi_{0}=0$ was studied in [76]; here, we generalize the aforementioned analysis by assuming that $\Phi_{0} \neq 0$. We also perform a more comprehensive analysis of the ensuing solutions by studying the different profiles of the coupling function, scalar field, and bulk potential, which emerge as the values of the parameters of the model vary. In addition, we supplement our analysis with the study of the effective theory on the brane and of the physical constraints imposed on the solutions. In order to simplify our notation, we set $\Phi_{1}=\xi \Phi_{0}$, where $\xi$ is a new integration constant. Then, Eq. (4.3) is written as

$\Phi(y)=\left\{\begin{array}{ll}\frac{\Phi_{0}}{2 \lambda}\left[\xi\left(\mu+e^{-k y}\right)^{\frac{2 \lambda}{1+4 \lambda}}-1\right], & \lambda \in \mathbb{R} \backslash\left\{-\frac{1}{4}, 0\right\} \\ \Phi_{0}\left(2+\xi e^{\mu e^{-k y}}\right), & \lambda=-\frac{1}{4}\end{array}\right\}$.

Substituting the above expression in Eq. (4.1), we obtain the following profile for the coupling function in terms of the extra coordinate

$f(y)=\left\{\begin{array}{ll}f_{0}+\frac{\Phi_{0}^{2}}{4 \lambda}\left[\xi^{2}\left(\mu+e^{-k y}\right)^{\frac{4 \lambda}{1+4 \lambda}}-1\right], & \lambda \in \mathbb{R} \backslash\left\{-\frac{1}{4}, 0\right\} \\ f_{0}+\Phi_{0}^{2}\left(1-\frac{\xi^{2}}{4} e^{2 \mu e^{-k y}}\right), & \lambda=-\frac{1}{4}\end{array}\right\}$.

The theory seems to contain five independent parameters: $f_{0}, \Phi_{0}, \lambda, \mu$, and $\xi$. However, the range of values for two of these will be constrained by the physical demands imposed on the model. To start with, both the scalar field $\Phi(y)$ and the coupling function $f(y)$ must be real and finite in their whole domain, according to the discussion in Sec. II. From Eq. (4.4), we observe that the allowed range of values of the parameter $\mu$ depends on the values that the parameter $\lambda$ assumes. In Appendix A, we consider in detail all possible values for $\lambda$ and the ensuing allowed ranges of values for $\mu$; the different cases and corresponding results are summarized in Table I. ${ }^{4}$

In addition, from the analysis of the previous section, it became clear that the theory is not robust unless a positive effective gravitational constant is obtained on the brane. This demand will impose a constraint on one of the remaining parameters of the theory: we choose this parameter to be $f_{0}$. Thus, in order to appropriately choose the values of $f_{0}$ to study the profile of the scalar field and coupling function, at this point we turn to the effective theory and compute the effective gravitational constant. We will employ Eq. (3.17), and consider separately the cases with $\lambda \neq-1 / 4$ and $\lambda=-1 / 4$. In the first case, using also Eq. (4.5), we obtain

$$
\begin{aligned}
\frac{1}{\kappa_{4}^{2}} & =2 \int_{0}^{\infty} d y e^{-2 k y} f(y) \\
& =\frac{1}{k}\left(f_{0}-\frac{\Phi_{0}^{2}}{4 \lambda}\right)+\frac{\Phi_{0}^{2} \xi^{2}}{2 \lambda} \int_{0}^{\infty} d y e^{-2 k y}\left(\mu+e^{-k y}\right)^{\frac{4 \lambda}{1+4 \lambda}} .
\end{aligned}
$$

\footnotetext{
${ }^{4}$ The symbol $\wedge$ that was used in Table I simply denotes the logical $A N D$. For example, the statement $A \wedge B$ is true if $A$ and $B$ are both true; else it is false.
} 
TABLE I. Range of values for all parameters of the model.

\begin{tabular}{ccc}
\hline \hline & Range of values for all parameters & \\
\hline$\xi \in \mathbb{R} \backslash\{0\}, \Phi_{0} \in \mathbb{R} \backslash\{0\}$, & $\lambda>0$ & $\mu \geq 0$ \\
$f_{0}$ is given by Eq. (4.12). & $\lambda \in\left(-\frac{1}{4}, 0\right) \wedge \frac{2 \lambda}{1+4 \lambda} \neq n, n \in \mathbb{Z}^{<}$ & $\mu>0$ \\
& $\lambda \in\left(-\frac{1}{4}, 0\right) \wedge \frac{2 \lambda}{1+4 \lambda}=n, n \in \mathbb{Z}^{<}$ & $\mu \in(-\infty,-1) \cup(0,+\infty)$ \\
& $\lambda=-1 / 4$ & $\mu \in(-\infty, 0) \cup(0,+\infty)$ \\
& $\lambda<-\frac{1}{4} \wedge \frac{2 \lambda}{1+4 \lambda} \neq n, n \in \mathbb{Z}^{>}$ & $\mu \geq 0$ \\
\hline \hline
\end{tabular}

In order to evaluate the integral on the right-hand side of the above equation, we perform the change of variable $t=e^{-k y}$. If we also use the integral representation of the hypergeometric function [83]

$$
\begin{aligned}
{ }_{2} F_{1} & (a, b ; c ; z) \\
= & \frac{\Gamma(c)}{\Gamma(b) \Gamma(c-b)} \int_{0}^{1} d t t^{b-1}(1-t)^{c-b-1}(1-z t)^{-a}, \\
& \quad \operatorname{Re}(c)>\operatorname{Re}(b)>0,
\end{aligned}
$$

we finally obtain the result:

$$
\begin{aligned}
\frac{1}{\kappa_{4}^{2}} & =\frac{M_{\mathrm{Pl}}^{2}}{8 \pi} \\
& =\frac{\Phi_{0}^{2}}{k}\left[\frac{f_{0}}{\Phi_{0}^{2}}-\frac{1}{4 \lambda}+\frac{\xi^{2} \mu^{\frac{4 \lambda}{1+4 \lambda}}}{4 \lambda}{ }_{2} F_{1}\left(-\frac{4 \lambda}{1+4 \lambda}, 2 ; 3 ;-\frac{1}{\mu}\right)\right] .
\end{aligned}
$$

We can further simplify the above expression using the following relations:

$$
{ }_{2} F_{1}(a, 2 ; 3 ; z)=\left\{\begin{array}{ll}
\frac{2(1-z)^{-a}\left[z(a+z-a z)+(1-z)^{a}-1\right]}{(a-2)(a-1) z^{2}}, & a \in \mathbb{R} \backslash\{1,2\} \\
\frac{2}{z^{2}}[-z-\ln (1-z)], & a=1 \\
\frac{2}{z^{2}(1-z)}[z+\ln (1-z)-z \ln (1-z)], & a=2
\end{array}\right\} .
$$

Then, for $\lambda \neq-1 / 4$, the four-dimensional effective Planck scale may be written in terms of elementary functions as follows:

$$
M_{\mathrm{Pl}}^{2}=\left\{\begin{array}{ll}
\frac{8 \pi \Phi_{0}^{2}}{k}\left\{\frac{f_{0}}{\Phi_{0}^{2}}-\frac{1}{4 \lambda}+\frac{(1+4 \lambda)^{2} \xi^{2}}{4 \lambda(1+6 \lambda)(1+8 \lambda)}\left[\mu^{\frac{2+12 \lambda}{1+4 \lambda}}-(1+\mu)^{\frac{1+8 \lambda}{1+4 \lambda}}\left(\mu-\frac{1+8 \lambda}{1+4 \lambda}\right)\right]\right\}, & \lambda \in \mathbb{R} \backslash\left\{-\frac{1}{4},-\frac{1}{6},-\frac{1}{8}, 0\right\} \\
\frac{8 \pi \Phi_{0}^{2}}{k}\left\{\frac{f_{0}}{\Phi_{0}^{2}}+2-4 \xi^{2}\left[1-\mu \ln \left(\frac{1+\mu}{\mu}\right)\right]\right\}, & \lambda=-\frac{1}{8} \\
\frac{8 \pi \Phi_{0}^{2}}{k}\left\{\frac{f_{0}}{\Phi_{0}^{2}}+\frac{3}{2}-\frac{3 \xi^{2}}{1+\mu}\left[-1+(1+\mu) \ln \left(\frac{1+\mu}{\mu}\right)\right]\right\}, & \lambda=-\frac{1}{6}
\end{array}\right\}
$$

On the other hand, for $\lambda=-1 / 4$, we readily obtain

$$
M_{\mathrm{Pl}}^{2}=\frac{8 \pi}{\kappa_{4}^{2}}=\frac{8 \pi \Phi_{0}^{2}}{k}\left\{\frac{f_{0}}{\Phi_{0}^{2}}+1-\frac{\xi^{2}}{8 \mu^{2}}\left[1+e^{2 \mu}(2 \mu-1)\right]\right\} .
$$

Since the effective four-dimensional gravitational scale $M_{P l}^{2}$ should be a positive number, Eqs. (4.10) and (4.11) impose the following constraints on the values of the ratio $f_{0} / \Phi_{0}^{2}$ :

$$
\begin{aligned}
& \frac{f_{0}}{\Phi_{0}^{2}}>\frac{1}{4 \lambda}\left\{1-\frac{(1+4 \lambda)^{2} \xi^{2}}{(1+6 \lambda)(1+8 \lambda)}\left[\mu^{\frac{2+12 \lambda}{1+4 \lambda}}-(1+\mu)^{\frac{1+8 \lambda}{1+4 \lambda}}\left(\mu-\frac{1+8 \lambda}{1+4 \lambda}\right)\right]\right\}, \quad \lambda \in \mathbb{R} \backslash\left\{-\frac{1}{4},-\frac{1}{6},-\frac{1}{8}, 0\right\} \\
& \frac{f_{0}}{\Phi_{0}^{2}}>-2\left\{1-2 \xi^{2}\left[1-\mu \ln \left(\frac{1+\mu}{\mu}\right)\right]\right\}, \quad \lambda=-\frac{1}{8}, \\
& \frac{f_{0}}{\Phi_{0}^{2}}>-\frac{3}{2}\left\{1-\frac{2 \xi^{2}}{1+\mu}\left[-1+(1+\mu) \ln \left(\frac{1+\mu}{\mu}\right)\right]\right\}, \quad \lambda=-\frac{1}{6}, \\
& \frac{f_{0}}{\Phi_{0}^{2}}>-1+\frac{\xi^{2}}{8 \mu^{2}}\left[1+e^{2 \mu}(2 \mu-1)\right], \quad \lambda=-\frac{1}{4} .
\end{aligned}
$$


We choose to use the above constraints in order to limit the range of values of the parameter $f_{0}$. The remaining parameters $\Phi_{0}, \lambda$, and $\xi$ may then take values in almost the entire set of real numbers, specifically $\Phi_{0} \in \mathbb{R} \backslash\{0\}$, $\xi \in \mathbb{R} \backslash\{0\}$, and $\lambda \in \mathbb{R} \backslash\{0\}$. These ranges of values are also summarized in Table I. We finally note that the above constraints for the positivity of the effective four-dimensional gravitational constant allow for both positive and negative values of the parameter $f_{0}$.

We now proceed to study the profile of our solution. In Fig. 4(a), we depict the form of the warp factor $e^{-2 k|y|}$ and the coupling function $f(y)$ in terms of the coordinate $y$ along the fifth dimension, for $\Phi_{0}=1, \lambda=1 / 7, \xi=1$, $\mu=1, k=1$, and $f_{0}=0$, which, as one can verify, is allowed by Eq. (4.12). The warp factor is always localized close to the brane and vanishes at the boundary of spacetime independently of the values of the parameters. The behavior of the coupling function though depends strongly on the values of the parameters of the model. For $\lambda>0$ and $\mu \geq 0$, the qualitative behavior of the coupling function is the same as the one that is illustrated in Fig. 4(a). In Fig. 4(b), we present the behavior of the coupling function for various values of the parameters $\mu$ and $\Phi_{0}$ while $\lambda$ is now in the regime $(-1 / 4,0)$. We note that, for generic values of the parameters, the asymptotic value of $f(y)$, as $y \rightarrow \infty$, is not zero: if so desired, one may choose $f_{0}$ to be equal to $\mathcal{C}_{\infty}$, which indicates the value that $f_{0}$ should have in order to get a vanishing coupling function at infinity; from Eq. (4.5), we can immediately calculate that $\mathcal{C}_{\infty}=\frac{\Phi_{0}^{2}}{4 \lambda}\left(1-\xi^{2} \mu^{\frac{4 \lambda}{1+4 \lambda}}\right)$. In Fig. 4(b), one can clearly see the strong dependence of the profile of the coupling function also on the value of the parameter $\mu$. As $\mu$ approaches zero,

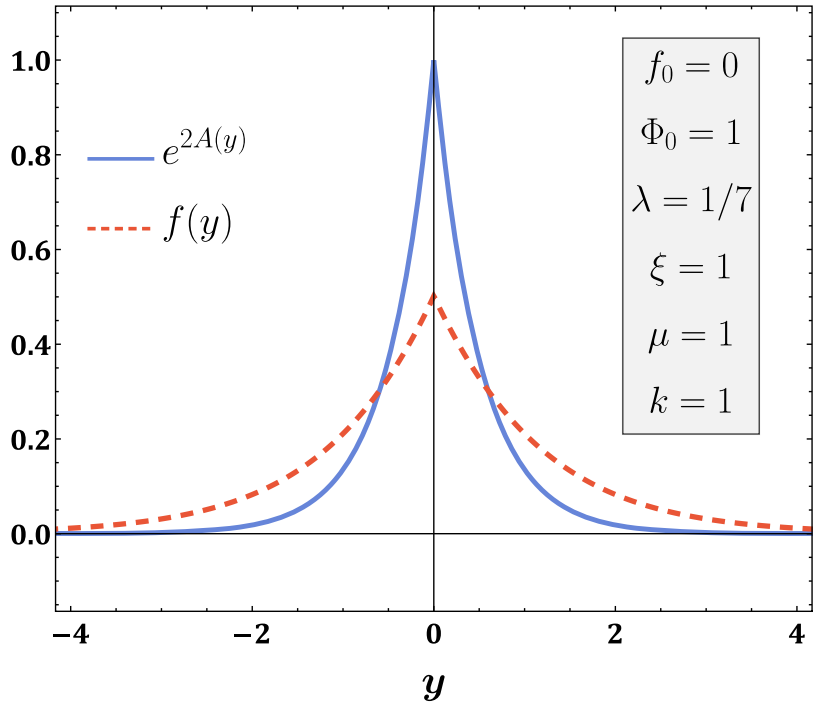

(a) the coupling function is characterized by a plateau around our brane; the closer the value of $\mu$ is to zero, the wider the plateau. On the contrary, $\Phi_{0}$ does not significantly affect the behavior of $f(y)$; it just scales the function as a whole. The behavior depicted in Fig. 4(b) holds for all values of $\lambda$ in the regime $\left(-\frac{1}{4}, 0\right)$ as long as $\mu>0$. A different behavior appears in the case where $\frac{2 \lambda}{1+4 \lambda}=-2 n, n \in \mathbb{Z}^{>}$, and $\mu<-1$; in this case the behavior of the coupling function is exactly the same as the one for $\lambda<-\frac{1}{4}$ and $\frac{2 \lambda}{1+4 \lambda} \neq n$, with $n \in \mathbb{Z}^{>}$, which will be discussed next.

In Fig. 5(a), we display the behavior of the coupling function $f(y)$ for $\Phi_{0}=1, \mu=1, k=1, \xi=1$, and values of $\lambda$ in the regime $\lambda<-1 / 4$. Since it holds that $\frac{2 \lambda}{1+4 \lambda} \neq n$, with $n \in \mathbb{Z}^{>}$, the parameter $\mu$ is constrained to values greater than or equal to zero. For easy comparison, the parameter $f_{0}$ has been taken to be equal to $\mathcal{C}_{0}$, which is the value that leads to $f(0)=0$; again, from Eq. (4.5), we find that $\mathcal{C}_{0}=\frac{\Phi_{0}^{2}}{4 \lambda}\left[1-\xi^{2}(\mu+1)^{\frac{4 \lambda}{1+4 \lambda}}\right]$. In such a model, the nonminimal coupling of the scalar field to the five-dimensional scalar curvature is nonvanishing in the bulk but disappears at the location of the brane. In this range of values for the parameter $\lambda$, the behavior of the coupling function, as depicted in Fig. 5(a), does not change regardless of the values of all the other parameters. In contrast, when $\lambda$ satisfies the condition $\frac{2 \lambda}{1+4 \lambda}=n$, the profile of the coupling function is extremely sensitive to changes in the parameter $\mu$. Indeed, Fig. 5(b) shows the behavior of the coupling function $f(y)$ for $k=1, \xi=1$ and $f_{0}=\mathcal{C}_{\infty}$, while $\lambda=$ $-1 / 3$ or $\frac{2 \lambda}{1+4 \lambda}=2$. In this figure, we focus on values of $\mu$ that are smaller than or equal to $-1 / 2$. We observe that, as $\mu$ approaches and exceeds -1 , the behavior of the coupling

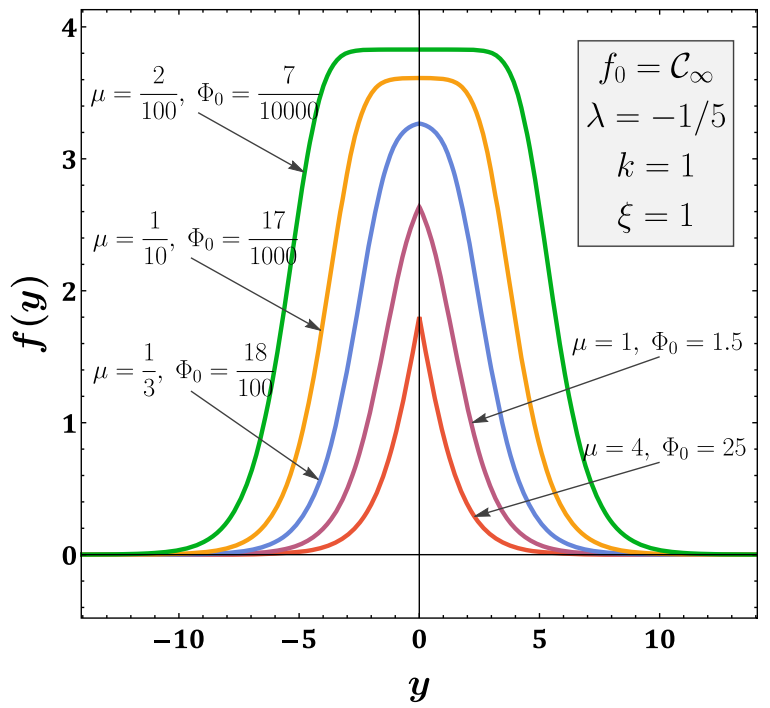

(b)

FIG. 4. (a) Warp factor $e^{2 A(y)}=e^{-2 k|y|}$ and coupling function $f(y)$ in terms of the coordinate $y$ for $\lambda=1 / 7$, and (b) the coupling function for $\lambda=-1 / 5$ in the regime $(-1 / 4,0)$ which satisfies $\frac{2 \lambda}{1+4 \lambda}=-2$ and different values of parameters $\mu$ and $\Phi_{0} . \mathcal{C}_{\infty}$ indicates the value that the parameter $f_{0}$ should have in order to get a vanishing coupling function $f(y)$ at $y \rightarrow \infty$. 


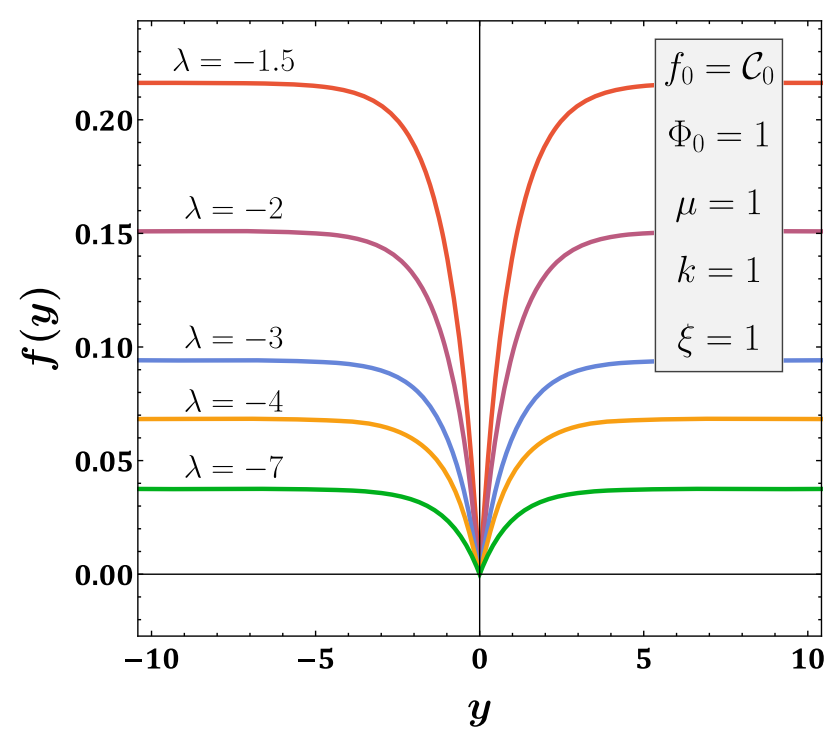

(a)

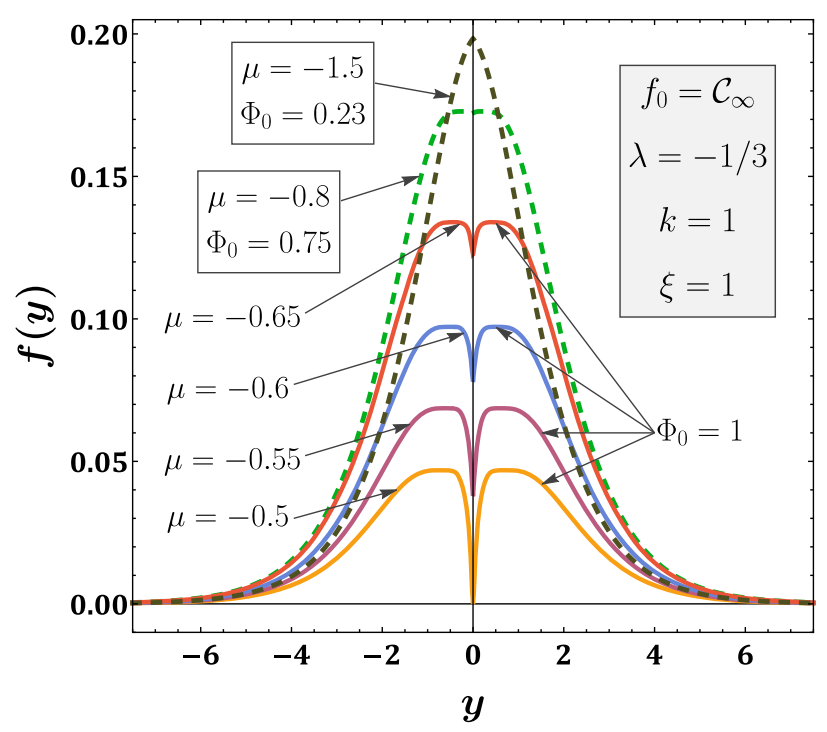

(b)

FIG. 5. (a) Coupling function $f(y)$ in terms of the coordinate $y$ for $\Phi_{0}=1, \mu=1, k=1, \xi=1$, and values of $\lambda$ smaller than $-\frac{1}{4}$ with $\frac{2 \lambda}{1+4 \lambda} \neq n, n \in \mathbb{Z}^{>}$. (b) Coupling function $f(y)$ for $\lambda=-1 / 3$, which satisfies $\frac{2 \lambda}{1+4 \lambda}=2, k=1, \xi=1$, and $f_{0}=\mathcal{C}_{\infty}$, while $\mu$ takes values equal or lower than $-1 / 2$.

function becomes similar to the one in Figs. 4(a) and 4(b). Here, we have chosen again $f_{0}=\mathcal{C}_{\infty}$; therefore, the nonminimal coupling takes its maximum value on or close to our brane while it vanishes at infinity. On the other hand, as $\mu$ approaches zero and takes on positive values, the profile of $f(y)$ resembles more the one depicted in Fig. 5(a).

Finally, in the case where $\lambda=-1 / 4$, the coupling function, as presented in Eq. (4.5), is given by a double exponential expression. It is not hard to realize that the qualitative behavior of $f(y)$ in this case is similar to the one in Fig. 4(b) when $\mu<0$ and similar to Fig. 5(a) when $\mu>0$. It is also necessary to stress that the behavior of the scalar field $\Phi(y)$ is similar to that of the coupling function $f(y)$, as one may easily conclude by observing Eqs. (4.4) and (4.5). Therefore, it is redundant to present any graphs of the scalar field as a function of the $y$ coordinate.

The scalar potential $V_{B}$ can be determined in terms of the extra dimension $y$ from Eq. (2.19) by substituting the function $f(y)$ given in Eq. (4.5). Consequently, we obtain

$$
V_{B}(y)=\left\{\begin{array}{ll}
-\Lambda_{5}-6 k^{2} f_{0}+\frac{3 k^{2} \Phi_{0}^{2}}{2 \lambda}-\frac{\xi^{2} k^{2} \Phi_{0}^{2}\left(\mu+e^{-k y}\right)^{-\frac{2+4 \lambda}{1+4 \lambda}}}{6 \lambda(1+4 \lambda)^{2}} & \\
\times\left\{\left[e^{-k y}(3+16 \lambda)+3(1+4 \lambda) \mu\right]^{2}-\lambda(3+16 \lambda) e^{-2 k y}\right\}, & \lambda \in \mathbb{R} \backslash\left\{-\frac{1}{4}, 0\right\} \\
-\Lambda_{5}-6 k^{2}\left(f_{0}+\Phi_{0}^{2}\right)+\frac{\xi^{2} \Phi_{0}^{2} k^{2}}{2} e^{2 \mu e^{-k y}}\left(3+4 \mu e^{-k y}+\mu^{2} e^{-2 k y}\right), & \lambda=-\frac{1}{4}
\end{array}\right\} .
$$

As in the linear case, it is possible to express the potential in terms of the scalar field $\Phi$ in closed form and obtain

$$
V_{B}(\Phi)=\left\{\begin{array}{ll}
-\Lambda_{5}-6 k^{2} f_{0}+\frac{3 k^{2} \Phi_{0}^{2}}{2 \lambda}+\frac{\xi^{2} k^{2} \Phi_{0}^{2}}{6 \lambda(1+4 \lambda)^{2}}\left(\frac{2 \lambda \Phi}{\xi \Phi_{0}}+\frac{1}{\xi}\right)^{-\frac{1+2 \lambda}{\lambda}\left\{3 \mu^{2} \lambda\right.} & \\
\left.+6 \lambda(3+16 \lambda) \mu\left(\frac{2 \lambda \Phi}{\xi \Phi_{0}}+\frac{1}{\xi}\right)^{\frac{1+4 \lambda}{2 \lambda}}-(3+16 \lambda)(3+15 \lambda)\left(\frac{2 \lambda \Phi}{\xi \Phi_{0}}+\frac{1}{\xi}\right)^{\frac{1+4 \lambda}{\lambda}}\right\}, & \lambda \in \mathbb{R} \backslash\left\{-\frac{1}{4}, 0\right\} \\
-\Lambda_{5}-6 k^{2}\left(f_{0}+\Phi_{0}^{2}\right)+\frac{\Phi_{0}^{2} k^{2}}{2}\left(\frac{\Phi}{\Phi_{0}}-2\right)^{2} \times & \\
\times\left\{3+4 \ln \left[\frac{1}{\xi}\left(\frac{\Phi}{\Phi_{0}}-2\right)\right]+\ln ^{2}\left[\frac{1}{\xi}\left(\frac{\Phi}{\Phi_{0}}-2\right)\right]\right\}, & \lambda=-1 / 4
\end{array}\right\} .
$$

In Figs. 6(a), 6(b), and 7(a), we display the behavior of the scalar potential $V_{B}$ as a function of the extra coordinate $y$ using the same values for the parameters as in Figs. 4(b), $5(\mathrm{a})$, and 5(b), respectively. It is worth observing the variety of forms that one may achieve for $V_{B}$ by varying the values of the parameters of the model. In Fig. 6(a), constructed for $\lambda \in(-1 / 4,0)$, the scalar potential adopts a negative value around the location of our brane, thus mimicking locally a 


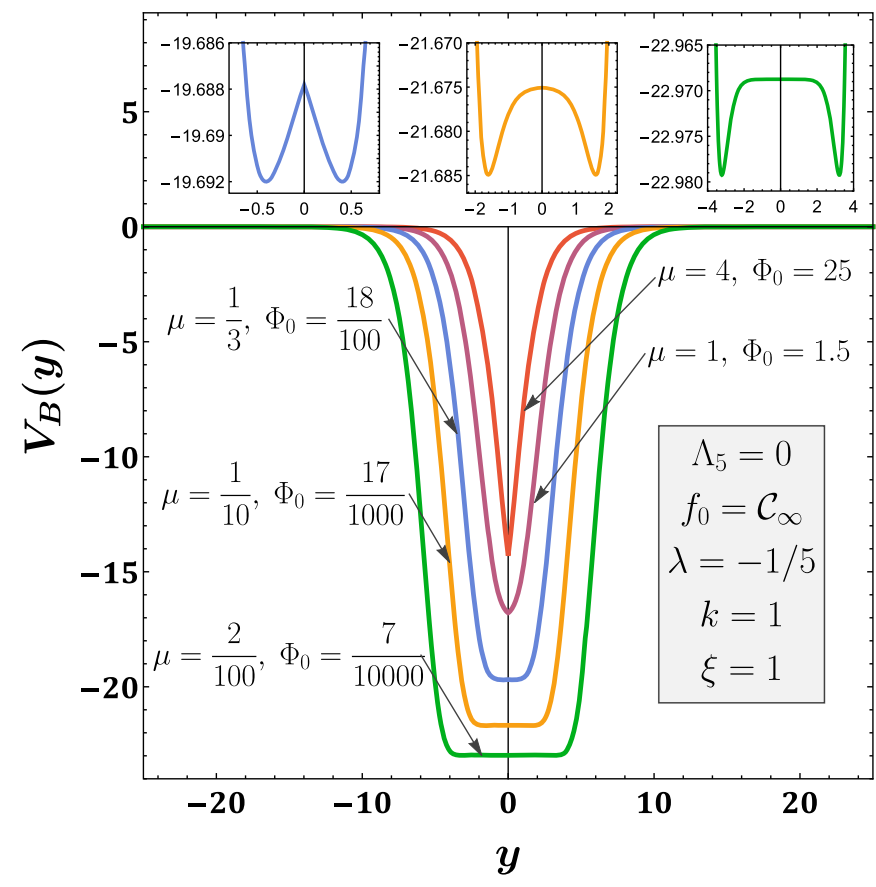

(a)

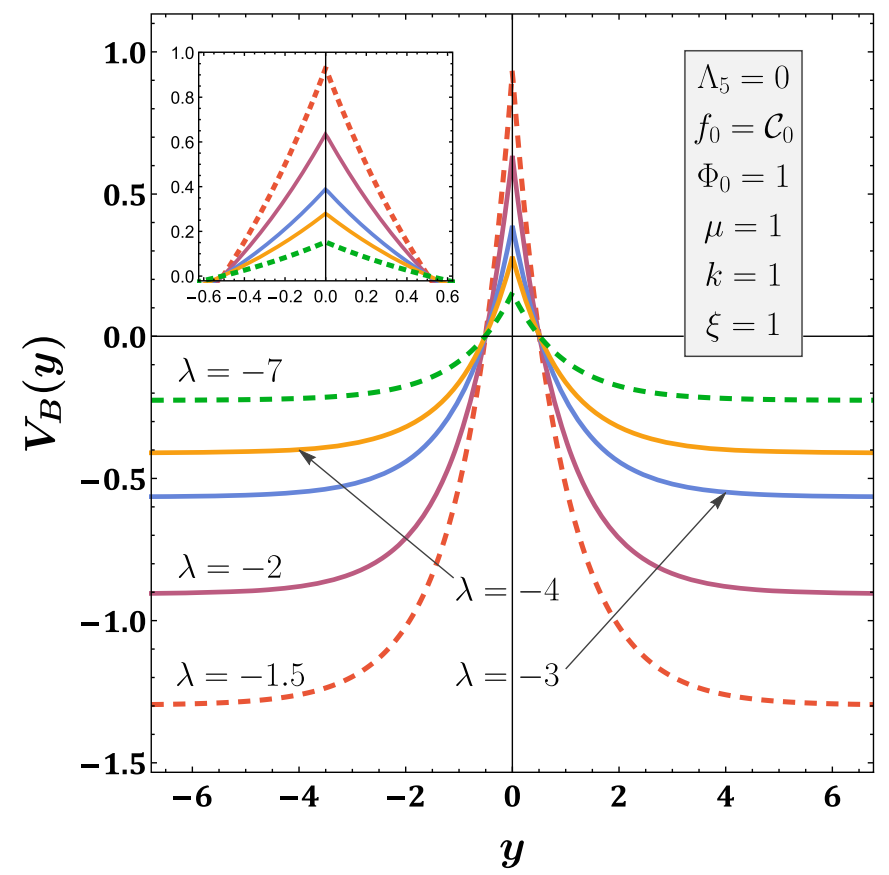

(b)

FIG. 6. The scalar potential $V_{B}$ in terms of the extra dimension $y$ for $\Lambda_{5}=0, k=1, \xi=1$, and (a) $\lambda=-1 / 5, f_{0}=\mathcal{C}_{\infty}$, and variable $\mu$ and $\Phi_{0}$, while in (b) $\Phi_{0}=1, \mu=1, f_{0}=\mathcal{C}_{0}$, and $\lambda=-1.5,-2,-3,-4,-7$. In each case, $\mathcal{C}_{\infty}$ and $\mathcal{C}_{0}$ should be evaluated separately.

negative bulk cosmological constant $\Lambda_{5}$, while it vanishes away from our brane. In Fig. 6(b), constructed for values of $\lambda$ in the regime $(-\infty,-1 / 4)$, the scalar potential has a positive value on and close to our brane and then decreases rapidly to a constant negative value, which depends on the values of the parameters of the theory. Finally, Fig. 7(a), constructed for $\lambda=-1 / 3$ and $\frac{2 \lambda}{1+4 \lambda}=2$, shows the sensitivity of the scalar potential to the value of parameter $\mu$ with

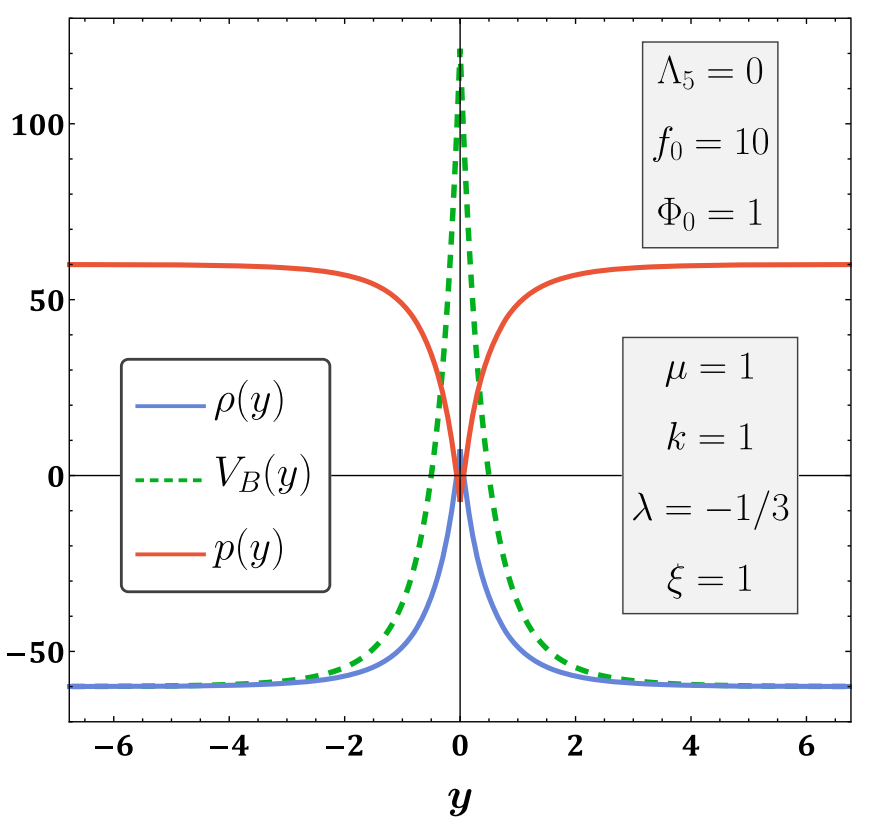

(b)

FIG. 7. (a) Scalar potential $V_{B}$ in terms of the extra dimension $y$ for $\Lambda_{5}=0, \lambda=-1 / 3, k=1, \xi=1$, and $f_{0}=\mathcal{C}_{\infty}$. The varying parameters are $\Phi_{0}$ and $\mu$. (b) Energy density $\rho$ and pressure $p$ of the system together with the scalar potential $V_{B}$ in terms of the coordinate $y$ for $\Lambda_{5}=0, f_{0}=10, \Phi_{0}=1, \mu=1, k=1, \xi=1$, and $\lambda=-1 / 3$. 
local minima and maxima appearing in its profile. It should be, however, stressed that the warp factor adopts its exponentially decaying form for all aforementioned profiles of the bulk potential and independently of whether $\Lambda_{5}=0$ or not.

The components of the energy-momentum tensor of the theory may be finally computed by employing Eqs. (3.6)(3.7). As in the linear case, we obtain

$$
\rho(y)=-p(y)=-6 k^{2} f(y), \quad p(y)=p^{i}(y)=p^{y}(y) .
$$

We discussed thoroughly in the previous section, that in order to satisfy the weak energy conditions on and close to the brane, we should allow the coupling function $f(y)$ to take negative values at these regimes. Thus, demanding that $f(0)<0$ and using Eq. (4.5), we obtain the constraints:

$$
\left\{\begin{array}{ll}
\frac{f_{0}}{\Phi_{0}^{2}}<\frac{1}{4 \lambda}\left[1-\xi^{2}(\mu+1)^{\left.\frac{4 \lambda}{1+4 \lambda}\right]},\right. & \lambda \in \mathbb{R} \backslash\left\{-\frac{1}{4}, 0\right\} \\
\frac{f_{0}}{\Phi_{0}^{2}}<\frac{\xi^{2}}{4} e^{2 \mu}-1, & \lambda=-\frac{1}{4}
\end{array}\right\} .
$$

In Fig. 7(b), we present the energy density $\rho(y)$, the pressure $p(y)=p^{i}(y)=p^{y}(y)$, and the scalar potential $V_{B}(y)$ in terms of the coordinate $y$. It is obvious that, for this particular set of parameters, chosen to satisfy the above constraints, the weak energy conditions are satisfied by the bulk matter on and close to the brane.

We should complete our bulk solution with the junction conditions introduced in the model due to the presence of the brane at $y=0$. As discussed in the previous section, the energy content of the brane is given by the combination $\sigma+V_{b}(\Phi)$, and it creates a discontinuity in the second derivatives of the warp factor, coupling function, and scalar field at the location of the brane. Using Eqs. (3.10) and (3.11), for $\lambda \neq\left\{-\frac{1}{4}, 0\right\}$ and $\lambda=-1 / 4$, we obtain

$$
\begin{aligned}
\sigma+\left.V_{b}(\Phi)\right|_{y=0}= & 6 k\left(f_{0}-\frac{\Phi_{0}^{2}}{4 \lambda}\right)+\frac{\Phi_{0}^{2} \xi^{2} k}{2 \lambda(1+4 \lambda)} \\
& \times[3(1+\mu)(1+4 \lambda)+4 \lambda](1+\mu)^{-\frac{1}{1+4 \lambda}}
\end{aligned}
$$

$\left.\partial_{\Phi} V_{b}\right|_{y=0}=\frac{2 k \xi \Phi_{0}}{1+4 \lambda}(1+\mu)^{-\frac{1+2 \lambda}{1+4 \lambda}}[4(1+\mu)(1+4 \lambda)-1]$,

and

$$
\begin{aligned}
\sigma+\left.V_{b}(\Phi)\right|_{y=0} & =6 k\left(f_{0}+\Phi_{0}^{2}\right)-\frac{\Phi_{0}^{2} \xi^{2} k e^{2 \mu}}{2}(3+2 \mu), \\
\left.\partial_{\Phi} V_{b}\right|_{y=0} & =-2 \Phi_{0} k \xi \mu e^{\mu}(\mu+2),
\end{aligned}
$$

respectively. Using the constraints (4.17) and (4.19), it is easy to deduce that in order to have a positive total energy density on the brane, namely $\sigma+\left.V_{b}(\Phi)\right|_{y=0}>0$, we should have, respectively

$$
\left\{\begin{array}{ll}
\frac{f_{0}}{\Phi_{0}^{2}}>\frac{1}{4 \lambda}\left\{1-\xi^{2}\left[(1+\mu)^{\frac{4 \lambda}{1+4 \lambda}}+\frac{4 \lambda}{3(1+4 \lambda)}(1+\mu)^{\left.-\frac{1}{1+4 \lambda}\right]}\right\},\right. & \lambda \in \mathbb{R} \backslash\left\{-\frac{1}{4}, 0\right\} \\
\frac{f_{0}}{\Phi_{0}^{2}}>-1+\frac{\xi^{2}}{12} e^{2 \mu}(3+2 \mu), & \lambda=-1 / 4
\end{array}\right\} .
$$

Let us also note that, from the constraint (4.20), we see that the brane interaction term $V_{b}$ can be a constant, and thus absorbed into the brane tension $\sigma$, under the condition $\mu=-2$. A similar fixing of the parameter $\mu$ follows from Eq. (4.18), which leads to the result $\mu=-\frac{3+4 \lambda}{4(1+4 \lambda)}$. However, in this case, care should be taken so that the resulting values of $\mu$, in terms of $\lambda$, are allowed by Table I.

The effective four-dimensional gravitational scale on the brane has already been calculated and is given in Eqs. (4.10) and (4.11). The effective cosmological constant on the brane $\Lambda_{4}$ can be calculated from Eq. (3.21), and is found to be zero also in this case, as anticipated.

\section{B. The energy conditions in the parameter space}

We will now study the inequalities (4.12), (4.16), and (4.21) and investigate again whether these may be simultaneously satisfied. In particular, we will study the parameter space between the ratio $f_{0} / \Phi_{0}^{2}$ and the parameters $\lambda, \mu$, and $\xi$. Given the large number of parameters, we will present three-dimensional graphs of the parameter space of the ratio $f_{0} / \Phi_{0}^{2}$ with two of the three parameters $\lambda, \mu, \xi$, while keeping the remaining one fixed. Before we continue, we elucidate that, in the forthcoming analysis, we will denote the right-hand side of inequality (4.12) with $F_{\text {eff }}(\lambda, \mu, \xi)$, since it is associated with the effective gravitational constant, the right-hand side of inequality (4.16), which refers to the energy conditions in the bulk, with $F_{B}(\lambda, \mu, \xi)$, and finally, the right-hand side of inequality (4.21), which involves the total energy density on the brane, with $F_{\mathrm{br}}(\lambda, \mu, \xi)$.

While pursuing to satisfy simultaneously all the aforementioned inequalities, we have performed a comprehensive study of the parameter space of the quantities $f_{0} / \Phi_{0}^{2}, \lambda$, $\mu$, and $\xi$ following the classification of cases, regarding the values of the free parameters, presented in Table I. We present the corresponding results below: 


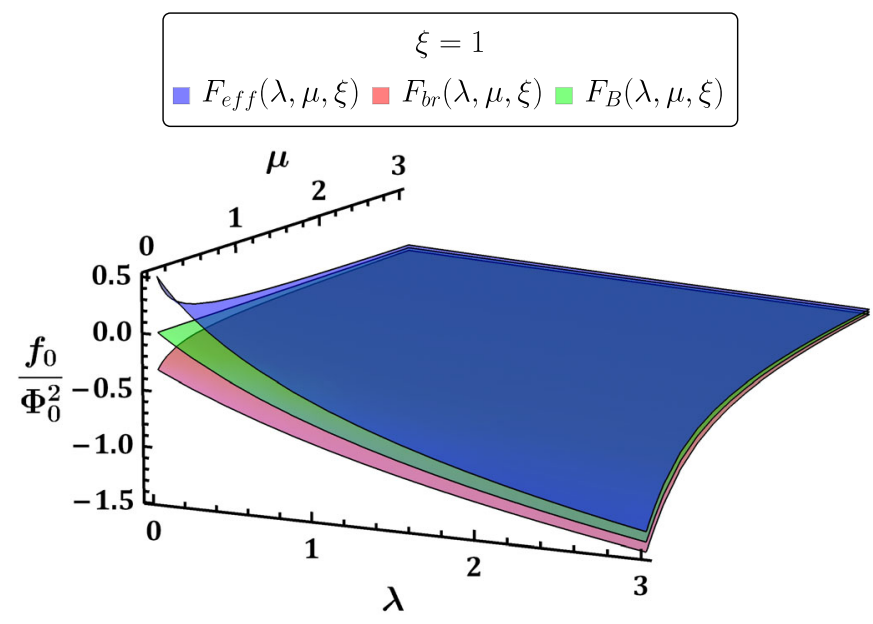

(a)

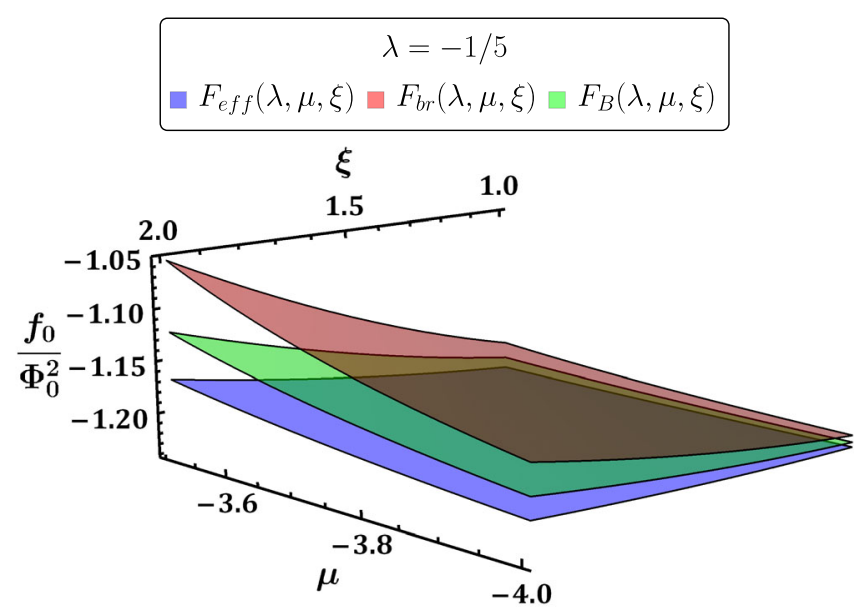

(b)

FIG. 8. (a) Parameter space of the quantities $f_{0} / \Phi_{0}^{2}, \lambda$ and $\mu$, for $\xi=1$ and $\lambda>0$. (b) Parameter space of the quantities $f_{0} / \Phi_{0}^{2}, \mu$ and $\xi$, for $\lambda=-1 / 5$ or $\frac{2 \lambda}{1+4 \lambda}=-2$ and $\mu<-1$. The graphs depict the functions $F_{\text {eff }}(\lambda, \mu, \xi), F_{\text {br }}(\lambda, \mu, \xi)$, and $F_{B}(\lambda, \mu, \xi)$.

(i) For $\lambda>0$, we have $\mu \geq 0$, while the parameter $\xi$ can take values in the whole set of real numbers except zero. In Fig. 8(a), we depict the parameter space of the quantities $f_{0} / \Phi_{0}^{2}, \lambda$, and $\mu$, for $\xi=1$ and $\lambda>0$. Although the surfaces representing the functions $F_{\text {eff }}(\lambda, \mu, \xi), \quad F_{b r}(\lambda, \mu, \xi)$, and $F_{B}(\lambda, \mu, \xi)$ change significantly for different values of the parameter $\xi$, their relative positions remain the same satisfying always the relation

$$
F_{\text {eff }}(\lambda, \mu, \xi)>F_{B}(\lambda, \mu, \xi)>F_{\text {br }}(\lambda, \mu, \xi)
$$

This means that there is no point in the parameter space for $\lambda>0$ at which all three inequalities are satisfied simultaneously. It is possible though to satisfy simultaneously the inequalities (4.12) and (4.21). Particularly, for every value of the ratio $f_{0} / \Phi_{0}^{2}$ which is greater than the value of the function $F_{\text {eff }}(\lambda, \mu, \xi)$ at any given point in the parameter space the aforementioned two inequalities will be satisfied. This means that the positivity of both the effective four-dimensional gravitational constant and the total energy density on the brane is ensured. In contrast, there is no point in the parameter space at which we can satisfy the inequality (4.16) because the surface of the function $F_{B}(\lambda, \mu, \xi)$ lies always below the surface of the function $F_{\text {eff }}(\lambda, \mu, \xi)$; as a result, the weak energy conditions are always violated by the bulk matter close to the brane.

(ii) For $\lambda \in\left(-\frac{1}{4}, 0\right), \frac{2 \lambda}{1+4 \lambda} \neq n, n \in \mathbb{Z}^{<}$, we have $\mu>0$, and we obtain the same qualitative behavior as in the previous case. However, when $\frac{2 \lambda}{1+4 \lambda}=n$, we have $\mu \in(-\infty,-1) \cup(0, \infty)$. In this case, the position of the surfaces $F_{\text {eff }}(\lambda, \mu, \xi), F_{b r}(\lambda, \mu, \xi)$, and $F_{B}(\lambda, \mu, \xi)$ are different in the region of the parameter space where $\mu<-1$ and in the region where $\mu>0$. Specifically, in this case we find that

$$
\begin{array}{cl}
F_{\text {br }}(\lambda, \mu, \xi)>F_{B}(\lambda, \mu, \xi)>F_{\text {eff }}(\lambda, \mu, \xi), & \mu<-1, \\
F_{\text {eff }}(\lambda, \mu, \xi)>F_{B}(\lambda, \mu, \xi)>F_{\text {br }}(\lambda, \mu, \xi), & \mu>0 .
\end{array}
$$

Again, there is no point in the parameter space at which we can satisfy simultaneously all inequalities. For $\mu>0$, the situation is similar to the one of case (i) depicted in Fig. 8(a). In this case, we may easily obtain a positive effective gravitational constant and a positive total energy density on the brane. For $\mu<-1$, though, as Fig. 8(b) also reveals, we have the choice of supplementing the positivity of the effective gravitational constant by either a positive total energy density on the brane or by a bulk matter that satisfies the energy conditions close to our brane.

(iii) For $\lambda=-1 / 4$, due to the different form of the solution, the functions $F_{\text {eff }}(-1 / 4, \mu, \xi), F_{\text {br }}(-1 / 4, \mu, \xi)$, and $F_{B}(-1 / 4, \mu, \xi)$ are given by different expressions. Now, these are found to satisfy the relations

$$
\begin{gathered}
F_{\text {eff }}(-1 / 4, \mu, \xi)>F_{B}(-1 / 4, \mu, \xi)>F_{\mathrm{br}}(-1 / 4, \mu, \xi), \\
\mu<0, \\
F_{\mathrm{br}}(-1 / 4, \mu, \xi)>F_{B}(-1 / 4, \mu, \xi)>F_{\mathrm{eff}}(-1 / 4, \mu, \xi), \\
\mu>0 .
\end{gathered}
$$

In this case, for $\mu<0$, we may obtain only the combination of a positive effective gravitational scale and a positive total energy density on the brane, in the region of the parameter space in which the value of the ratio $f_{0} / \Phi_{0}^{2}$ is greater than the value of the function $F_{\text {eff }}(-1 / 4, \mu, \xi)$; the relative positions of the different 
surfaces are the same as in Fig. 8(a). On the other hand, for $\mu>0$, we again have the choice of satisfying either Eqs. (4.12) and (4.16), in the region where $F_{\text {eff }}(-1 / 4, \mu, \xi)<f_{0} / \Phi_{0}^{2}<F_{B}(-1 / 4, \mu, \xi)$, or Eqs. (4.12) and (4.21), in the region where $f_{0} / \Phi_{0}^{2}>F_{\mathrm{br}}(-1 / 4, \mu, \xi)$. This situation is in turn similar to the one depicted in Fig. 8(b).

(iv) For $\lambda<-1 / 4, \frac{2 \lambda}{1+4 \lambda} \neq n, n \in \mathbb{Z}^{>}$, and for every allowed value of the parameters $\mu \geq 0$ and $\xi \in \mathbb{R} \backslash\{0\}$, we always have

$$
F_{\text {br }}(\lambda, \mu, \xi)>F_{B}(\lambda, \mu, \xi)>F_{\text {eff }}(\lambda, \mu, \xi) .
$$

In this case, the situation is similar to the one depicted in Fig. 8(b), and we have again the choice of combining a positive effective gravitational constant with either a positive energy density on the brane or a bulk matter that satisfies the weak energy conditions close to and on our brane.

(v) For $\lambda<-1 / 4$ and $\frac{2 \lambda}{1+4 \lambda}=n, n \in \mathbb{Z}^{>}$, the parameter $\mu$ is free to take values in the whole set of real numbers. In Figs. 9(a) and 9(b), we depict the parameter space of the ratio $f_{0} / \Phi_{0}^{2}$ and $\mu$ together with the curves of the functions $F_{\text {eff }}(\lambda, \mu, \xi)$, $F_{\text {br }}(\lambda, \mu, \xi)$, and $F_{B}(\lambda, \mu, \xi)$. Note that, for clarity of the graph, we have fixed the values of two parameters, i.e., $\lambda=-1 / 3$ and $\xi=1$, and thus present a two-dimensional graph. However, the situation remains the same for every other allowed value of the parameters $\lambda$ and $\xi$. We observe that

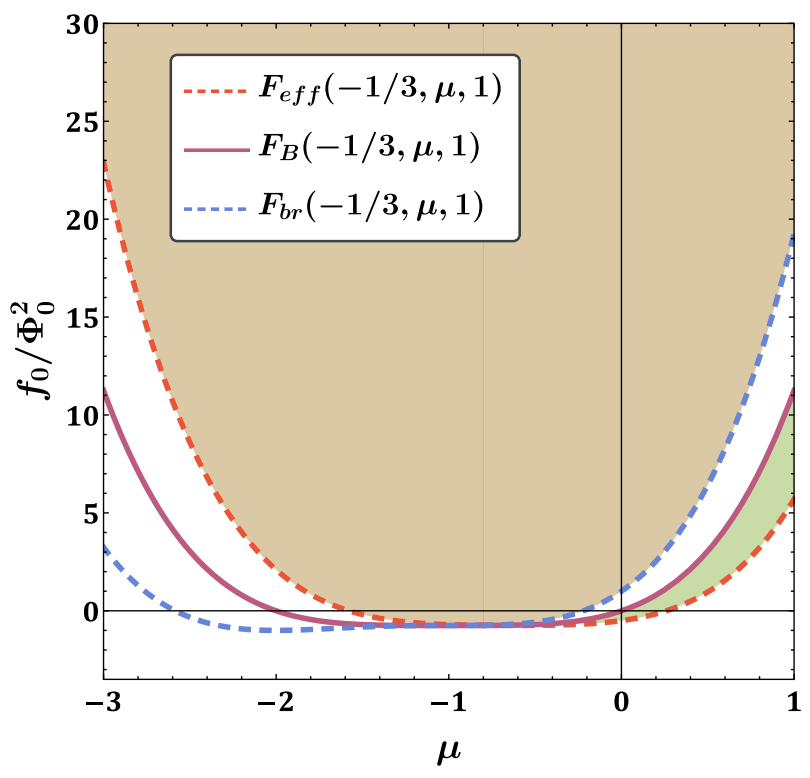

(a) there always exists a region in the parameter space in which we can have a positive value for the effective four-dimensional gravitation scale and satisfy the weak energy conditions close to the brane (green region) and a region in which both the four-dimensional gravitational constant and the total energy density on the brane are positive (brown region). Since there is no overlapping between the green and brown regions, as Fig. 9(b) reveals, there is no point in the parameter space where all three conditions are satisfied. For comparison, we note that the parameters in Fig. 7(b) have been chosen so that the depicted solution falls into the green area of Fig. 9(a).

\section{AN INVERSE-POWER COUPLING FUNCTION IN TERMS OF $y$}

In this and the following two sections, we will consider explicit forms of the coupling function $f(y)$ in terms of the coordinate $y$. These forms cannot be easily expressed in terms of the scalar field $\Phi$ in a closed form; they are however legitimate choices that satisfy the reality and finiteness conditions imposed in Sec. II. We start with the following expression:

$$
f(y)=f_{0}+\frac{\Phi_{0}^{2}}{k^{\lambda}\left(y+y_{0}\right)^{\lambda}},
$$

where $\left(f_{0}, \Phi_{0}\right) \in \mathbb{R} \backslash\{0\}$ while $\left(\lambda, y_{0}\right) \in(0,+\infty)$. The factor $k^{\lambda}$ in the denominator was introduced to make the product $k\left(y+y_{0}\right)$ dimensionless.

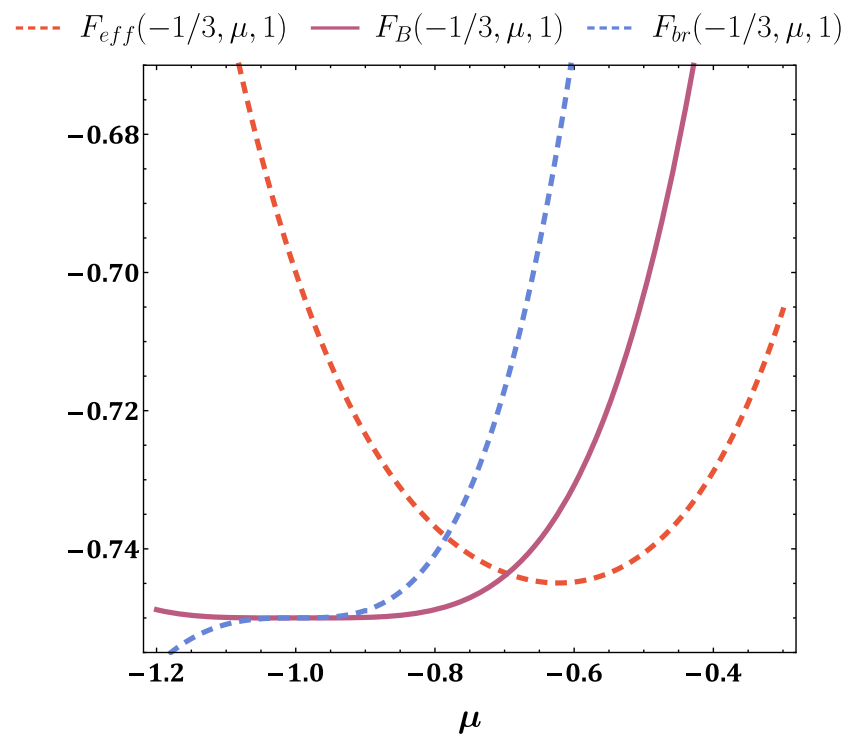

(b)

FIG. 9. (a) Parameter space of the quantities $f_{0} / \Phi_{0}^{2}$ and $\mu$, for $\xi=1, \lambda=-1 / 3$. (b) Magnification of a particular region of the previous figure in order to get a clear picture of the behavior of the functions $F_{\text {eff }}(-1 / 3, \mu, 1), F_{\mathrm{br}}(-1 / 3, \mu, 1)$, and $F_{B}(-1 / 3, \mu, 1)$ close to $\mu=-0.8$. 


\section{A. The bulk solution}

Substituting the aforementioned coupling function in Eq. (2.18) we obtain the differential equation:

$$
\left[\Phi^{\prime}(y)\right]^{2}=\frac{\lambda \Phi_{0}^{2}}{k^{\lambda}\left(y+y_{0}\right)^{\lambda+2}}\left[k\left(y+y_{0}\right)-\lambda-1\right] .
$$

The right-hand side of the above equation should be always positive; evaluating at $y=0$, the above yields the following constraint on the parameters of the model:

$$
\frac{k y_{0}}{\lambda+1}>1
$$

The function $\left[\Phi^{\prime}(y)\right]^{2}$ could, in principle, be zero at the point where $\Phi(y)$ has an extremum. However, from Eq. (5.2) this may happen only at $y=y_{0}\left(\frac{\lambda+1}{k y_{0}}-1\right)$ which, upon using the constraint (5.3), turns out to be negative. Therefore, the scalar field does not have an extremum in the whole domain $y \in[0,+\infty)$, which also means that $\Phi(y)$ is an one-to-one function in the same regime. In addition, from Eq. (5.2) it is straightforward to deduce that, as $y \rightarrow+\infty$, the physical constraint (2.23) is satisfied, thus, the scalar field does not diverge at infinity.

Let us now determine the explicit expression of the scalar field $\Phi(y)$ from Eq. (5.2). For simplicity and without loss of generality, we will assume that $\Phi_{0} \in(0,+\infty)$. Then, after taking the square root of Eq. (5.2), we have

$\Phi_{ \pm}(y)= \pm \frac{\Phi_{0} \sqrt{\lambda(\lambda+1)}}{k^{\lambda / 2}} \int d y\left(y+y_{0}\right)^{-\frac{\lambda}{2}-1}\left[\frac{k\left(y+y_{0}\right)}{\lambda+1}-1\right]^{\frac{1}{2}}$.

Setting $u=\frac{k\left(y+y_{0}\right)}{\lambda+1}$ and then $w=1-\frac{1}{u}$, the above integral takes the form

$$
\begin{aligned}
& \int d y\left(y+y_{0}\right)^{-\frac{\lambda}{2}-1}\left[\frac{k\left(y+y_{0}\right)}{\lambda+1}-1\right]^{\frac{1}{2}} \\
& =\left(\frac{k}{\lambda+1}\right)^{\frac{\lambda}{2}} \int d w(1-w)^{\frac{\lambda}{2}-\frac{3}{2}} w^{\frac{1}{2}} \\
& =\left(\frac{k}{\lambda+1}\right)^{\frac{\lambda}{2}} \int_{0}^{w} d t^{\frac{1}{2}}(1-t)^{\frac{\lambda}{2}-\frac{3}{2}}+C_{1} \\
& =\left(\frac{k}{\lambda+1}\right)^{\frac{\lambda}{2}} w^{\frac{3}{2}} \int_{0}^{1} d t^{\prime} t^{\prime \frac{1}{2}}\left(1-w t^{\prime}\right)^{\frac{\lambda}{2}-\frac{3}{2}}+C_{1},
\end{aligned}
$$

where in the last line we have made the change of variable $t^{\prime}=\frac{t}{w}$. Using the integral representation of the hypergeometric function (4.7), Eq. (5.4) leads to the following expression for the scalar field $\Phi(y)$ :

$$
\begin{aligned}
\Phi_{ \pm}(y)= & \pm \frac{2 \Phi_{0}}{3} \sqrt{\frac{\lambda}{(\lambda+1)^{\lambda-1}}}\left[1-\frac{\lambda+1}{k\left(y+y_{0}\right)}\right]^{\frac{3}{2}} \\
& \times{ }_{2} F_{1}\left(\frac{3}{2}-\frac{\lambda}{2}, \frac{3}{2} ; \frac{5}{2} ; 1-\frac{\lambda+1}{k\left(y+y_{0}\right)}\right) .
\end{aligned}
$$

In the above, we have also used the translational symmetry of the gravitational field equations with respect to the scalar field, discussed also in Sec. III A, to set $C_{1}=0$.

A solution for the scalar field similar to Eq. (5.6) was derived in the context of our previous analysis [73] for an exponential coupling function $f(y)$ and an anti-de Sitter brane $(\Lambda<0)$. The mathematical properties of the solution were studied there in detail; therefore, here we adapt those results in the present case and present our solutions for the scalar field without repeating the analysis-we refer the interested reader to our previous work for further information.

Trying to simplify Eq. (5.6), we first note that for every value of the coordinate $y$, the argument $1-\frac{\lambda+1}{k\left(y+y_{0}\right)}$ of the hypergeometric function is positive and smaller than unity. Therefore, one can expand the hypergeometric function in power series as $[73,83]$

$$
\begin{aligned}
{ }_{2} & F_{1}\left(\frac{3}{2}-\frac{\lambda}{2}, \frac{3}{2} ; \frac{5}{2} ; 1-\frac{\lambda+1}{k\left(y+y_{0}\right)}\right) \\
& =\sum_{n=0}^{\infty} \frac{\Gamma\left(\frac{3}{2}-\frac{\lambda}{2}+n\right)}{\Gamma\left(\frac{3}{2}-\frac{\lambda}{2}\right)} \frac{3}{(2 n+3) n !}\left[1-\frac{\lambda+1}{k\left(y+y_{0}\right)}\right]^{n} .
\end{aligned}
$$

There are two interesting categories of values for the parameter $\lambda$ which lead to even simpler and more elegant expressions for the hypergeometric function and subsequently for the scalar field. These are as follows:

(i) If $\lambda=1+2 q$ with $q \in \mathbb{Z}^{>}$, then, from Eq. (5.7), we have

$$
{ }_{2} F_{1}\left(\frac{3-\lambda}{2}, \frac{3}{2} ; \frac{5}{2} ; 1-\frac{\lambda+1}{k\left(y+y_{0}\right)}\right)=\left\{\begin{array}{cc}
1, & q=1 \\
1+\sum_{n=1}^{q-1} \frac{3(-q+1)(-q+2) \cdots(-q+n)}{(2 n+3) n !}\left[1-\frac{\lambda+1}{k\left(y+y_{0}\right)}\right]^{n}, & q>1
\end{array}\right\} .
$$


The solution for the scalar field then easily follows by using Eqs. (5.6) and (5.8) and substituting the selected values for the parameter $\lambda$ (or $q$ ). As indicative cases, we present below the form of the scalar field for $^{5} \lambda=3$ (i.e., $q=1$ )

$$
\Phi_{ \pm}(y)= \pm \frac{\Phi_{0}}{2 \sqrt{3}}\left[1-\frac{4}{k\left(y+y_{0}\right)}\right]^{3 / 2}
$$

and $\lambda=5$ (i.e., $q=2$ )

$$
\begin{aligned}
\Phi_{ \pm}(y)= & \pm \frac{\Phi_{0} \sqrt{5}}{54}\left[1-\frac{6}{k\left(y+y_{0}\right)}\right]^{3 / 2} \\
& \times\left\{1-\frac{3}{5}\left[1-\frac{6}{k\left(y+y_{0}\right)}\right]\right\} .
\end{aligned}
$$

(ii) If $\lambda=2 q$ with $q \in \mathbb{Z}^{>}$, we can always express the hypergeometric function in Eq. (5.6) in terms of elementary functions, namely arcsin, square roots, and powers of its argument. For $\lambda=2$ (i.e.. $q=1$ ), it is

$$
{ }_{2} F_{1}\left(\frac{1}{2}, \frac{3}{2} ; \frac{5}{2} ; u^{2}\right)=\frac{3}{2} \frac{1}{u^{2}}\left(\frac{\arcsin u}{u}-\sqrt{1-u^{2}}\right) .
$$

Therefore, from Eq. (5.6), the scalar field for $\lambda=2$ can be written in the form

$$
\begin{aligned}
\Phi_{ \pm}(y)= & \pm \frac{\Phi_{0} \sqrt{2}}{3}\left[1-\frac{3}{k\left(y+y_{0}\right)}\right]^{\frac{1}{2}}\left[\left(1-\frac{3}{k\left(y+y_{0}\right)}\right)^{-\frac{1}{2}}\right. \\
& \left.\times \arcsin \left(\sqrt{1-\frac{3}{k\left(y+y_{0}\right)}}\right)-\sqrt{\frac{3}{k\left(y+y_{0}\right)}}\right] .
\end{aligned}
$$

For larger values of $\lambda$ (i.e., for $q=1+\ell$, with $\left.\ell \in \mathbb{Z}^{>}\right)$, the following relation holds:

$$
\begin{aligned}
& { }_{2} F_{1}\left(\frac{1}{2}-\ell, \frac{3}{2} ; \frac{5}{2} ; u^{2}\right) u^{2} \\
& =\alpha\left(\frac{\arcsin u}{u}-\sqrt{1-u^{2}}\right) \\
& +\sqrt{1-u^{2}}\left(\beta_{1} u^{2}+\beta_{2} u^{4}+\cdots+\beta_{\ell-1} u^{2(\ell-1)}+\beta_{\ell} u^{2 \ell}\right),
\end{aligned}
$$

\footnotetext{
${ }^{5}$ For completeness, we present here also the solution for the limiting case with $\lambda=1$ (i.e., for $q=0$ ); this has the form

$$
\Phi_{ \pm}(y)= \pm \frac{2 \Phi_{0}}{3}\left[\operatorname{arctanh}\left(\sqrt{1-\frac{2}{k\left(y+y_{0}\right)}}\right)-\sqrt{1-\frac{2}{k\left(y+y_{0}\right)}}\right] .
$$
}

where $\alpha, \beta_{1}, \ldots, \beta_{\ell}$ are constant coefficients, which satisfy a system of $\ell+1$ linear algebraic equations [73] - the solution of this system readily determines the unknown coefficients $\alpha, \beta_{1}, \ldots, \beta_{\ell}$. For example, for $\ell=1$ (i.e., for $q=2$, or equivalently $\lambda=4$ ), this set of equations gives $\alpha=3 / 8$ and $\beta_{1}=3 / 4$. Upon substituting these in (5.10), the solution for the scalar field follows from Eq. (5.6) and has the form

$$
\begin{aligned}
\Phi_{ \pm}(y)= & \pm \frac{\Phi_{0}}{5 \sqrt{5}}\left[1-\frac{5}{k\left(y+y_{0}\right)}\right]^{\frac{1}{2}} \\
& \times\left[\frac{1}{2}\left(1-\frac{5}{k\left(y+y_{0}\right)}\right)^{-\frac{1}{2}}\right. \\
& \times \arcsin \left(\sqrt{1-\frac{5}{k\left(y+y_{0}\right)}}\right) \\
& \left.+\sqrt{\frac{5}{k\left(y+y_{0}\right)}}\left(\frac{1}{2}-\frac{5}{k\left(y+y_{0}\right)}\right)\right] .
\end{aligned}
$$

In Fig. 10, we depict the coupling function $f(y)$ and the scalar field $\Phi(y)$ for the indicative set of parameters $f_{0}=0, \Phi_{0}=1.5, y_{0}=1, k=2.5$, and $\lambda=1.5$. For comparison, we also display the exponentially decreasing warp factor. The coupling function remains localized near the brane and asymptotically decreases to the constant value $f_{0}$, which here has been taken to be zero. The scalar field starts from a constant value at the location of the brane, which for this set of parameters turns out to be zero, and goes asymptotically to a constant value that depends on the values of $\Phi_{0}$ and $\lambda$. Although this is not very clear from

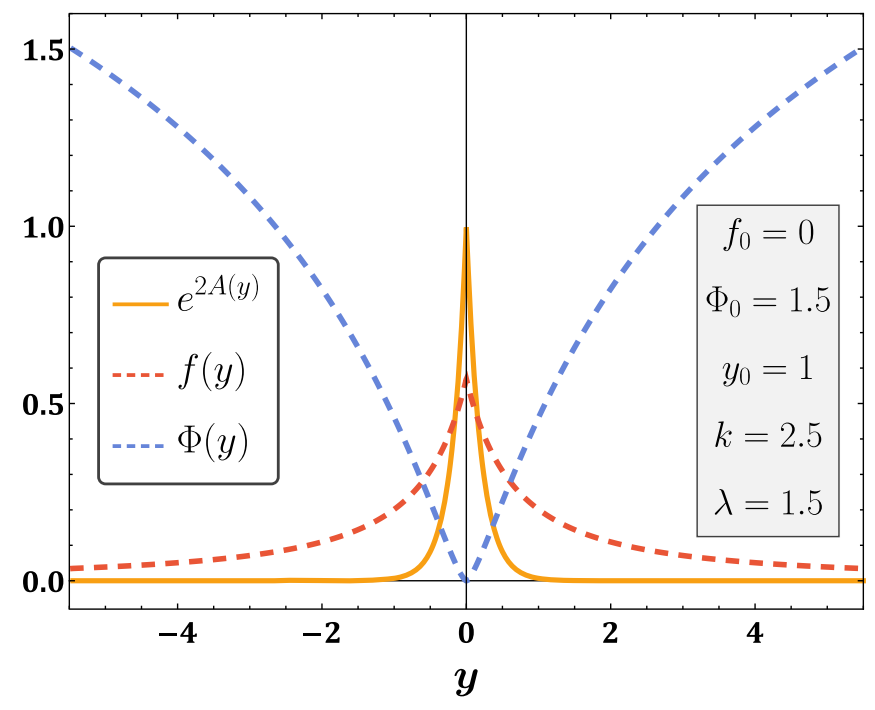

FIG. 10. Warp factor $e^{2 A(y)}=e^{-2 k|y|}$, the coupling function $f(y)$ and the scalar field $\Phi(y)$ in terms of the coordinate $y$ for $f_{0}=0, \Phi_{0}=1.5, y_{0}=1, k=2.5$, and $\lambda=1.5$. 
Fig. 10, it easily follows from Eq. (5.6) with the asymptotic value of the scalar field, as $y \rightarrow \pm \infty$, coming out to be

$$
\lim _{y \rightarrow \pm \infty} \Phi_{ \pm}(y)= \pm \frac{\sqrt{\pi} \Phi_{0}}{2} \sqrt{\frac{\lambda}{(\lambda+1)^{\lambda-1}}} \frac{\Gamma\left(\frac{\lambda}{2}-\frac{1}{2}\right)}{\Gamma\left(\frac{\lambda}{2}+1\right)} .
$$

It is worth noting that the profiles of both $f(y)$ and $\Phi(y)$ do not change with the variation of the values of the parameters.

The potential of the scalar field $V_{B}(y)$ in the bulk can be determined from Eq. (2.19) using the expression of the coupling function $f(y)$. Thus, we find

$$
\begin{aligned}
V_{B}(y)= & -\Lambda_{5}-6 k^{2} f_{0}-\frac{\Phi_{0}^{2}}{2 k^{\lambda}\left(y+y_{0}\right)^{\lambda+2}} \\
& \times\left[12 k^{2}\left(y+y_{0}\right)^{2}+7 \lambda k\left(y+y_{0}\right)+\lambda(\lambda+1)\right] .
\end{aligned}
$$

Since $\lambda>0$, the last term in the above expression is negative-definite; it also vanishes as $y \rightarrow+\infty$ leaving the parameters $\Lambda_{5}$ and $f_{0}$ to determine its asymptotic value. Depending on the values of the parameters, the potential may be either positive or negative at the location of the brane or asymptotically far away. In Fig. 11, one can observe the aforementioned behavior of the scalar potential $V_{B}(y)$. The values of the fixed parameters $\Lambda_{5}, f_{0}, \Phi_{0}, y_{0}, k$ are the same as in Fig. 10, while the parameter $\lambda$ varies.

The energy density $\rho(y)$ and pressure $p(y)=p^{i}(y)=$ $p^{y}(y)$ may be finally computed by employing Eqs. (3.6) and (3.7). Then, we are led to the result

$\rho(y)=-p(y)=-6 k^{2} f(y)=-6 k^{2}\left[f_{0}+\frac{\Phi_{0}^{2}}{k^{\lambda}\left(y+y_{0}\right)^{\lambda}}\right]$.

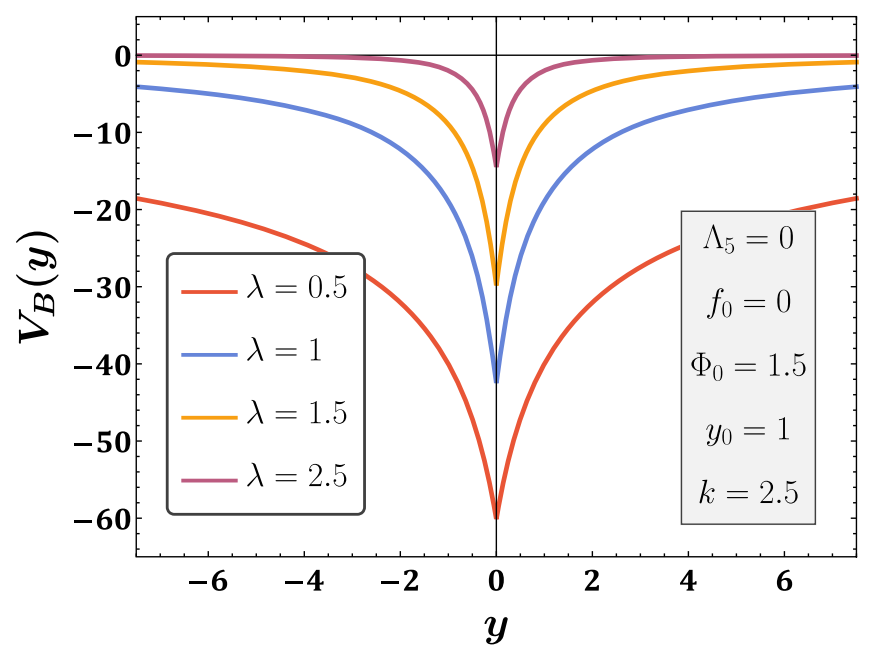

FIG. 11. Scalar potential $V_{B}$ in terms of the extra dimension $y$ for $\Lambda_{5}=0, f_{0}=0, \Phi_{0}=1.5, \quad y_{0}=1, \quad k=2.5$, and $\lambda=$ $0.5,1,1.5,2.5$ (from bottom to top).
If we wish to satisfy the weak energy conditions close and on the brane, we should have $\rho(0)>0$, which in turn means $f(0)<0$; in that case, the parameters of the model should satisfy the following inequality:

$$
\frac{f_{0}}{\Phi_{0}^{2}}<-\frac{1}{\left(k y_{0}\right)^{\lambda}}
$$

\section{B. Junction conditions and the effective theory}

From the field equations (2.12) and (2.14), we obtain the following junction conditions for the matter on the brane:

$$
\begin{aligned}
3 f(y)\left[A^{\prime}\right] & =-\left[f^{\prime}\right]-\left(\sigma+V_{b}\right), \\
{\left[\Phi^{\prime}\right] } & =4\left[A^{\prime}\right] \partial_{\Phi} f+\partial_{\Phi} V_{b},
\end{aligned}
$$

where all quantities are again evaluated at $y=0$. The only difference in this case is that we have used the derivatives of the coupling function with respect to the coordinate $y$ rather than the one with respect to the scalar field. This is due to the fact that the explicit expression of the function $f(\Phi)$ is not known-although $\Phi(y)$ is a one-to-one function, it cannot in general be inverted. Taking advantage of the $\mathbf{Z}_{2}$ symmetry in the bulk, we can easily evaluate the total energy density on the brane by Eq. (5.17), which is given by

$$
\begin{aligned}
\sigma+\left.V_{b}(\Phi)\right|_{y=0} & =6 k f_{0}+\frac{2 \Phi_{0}^{2}}{k^{\lambda} y_{0}^{\lambda+1}}\left(3 k y_{0}+\lambda\right) \\
& =6 k \Phi_{0}^{2}\left[\frac{f_{0}}{\Phi_{0}^{2}}+\frac{3 k y_{0}+\lambda}{3\left(k y_{0}\right)^{\lambda+1}}\right] .
\end{aligned}
$$

If we demand the total energy density on the brane to be positive, namely $\sigma+\left.V_{b}(\Phi)\right|_{y=0}>0$, then we straightforwardly deduce the constraint

$$
\frac{f_{0}}{\Phi_{0}^{2}}>-\frac{3 k y_{0}+\lambda}{3\left(k y_{0}\right)^{\lambda+1}} .
$$

In order to evaluate the first jump condition (5.16), we write $\partial_{\Phi} f=\partial_{y} f / \Phi^{\prime}$ and $\partial_{\Phi} V_{b}=\partial_{y} V_{b} / \Phi^{\prime}$. We are allowed to do this since, as we mentioned previously, the function $\Phi(y)$ does not possess any extrema in the bulk; therefore, $\Phi^{\prime}(y)$ never vanishes. Then, multiplying both sides of Eq. (5.16) by $\Phi^{\prime}$ and using Eq. (5.2), we obtain the condition

$$
\left.\partial_{y} V_{b}\right|_{y=0}=-\frac{2 \lambda \Phi_{0}^{2}}{k^{\lambda} y_{0}^{\lambda+2}}\left(3 k y_{0}+\lambda+1\right) .
$$

Due to the fact that $\lambda>0, k>0$, and $y_{0}>0$, the right-hand side of the above equation never vanishes, which means that $V_{b} \neq$ const.

Let us now focus on the effective four-dimensional theory on the brane. Using Eq. (3.17) and the expression for the coupling function, from Eq. (5.1) we obtain 


$$
\begin{aligned}
\frac{1}{\kappa_{4}^{2}} & =\frac{f_{0}}{k}+\frac{2 \Phi_{0}^{2}}{k^{\lambda}} \int_{0}^{\infty} d y \frac{e^{-2 k y}}{\left(y+y_{0}\right)^{\lambda}} \\
& =\frac{f_{0}}{k}+\frac{2 \Phi_{0}^{2} e^{2 k y_{0}}}{k^{\lambda}} \int_{0}^{\infty} d y \frac{e^{-2 k\left(y+y_{0}\right)}}{\left(y+y_{0}\right)^{\lambda}}
\end{aligned}
$$

Setting $t=2 k\left(y+y_{0}\right)$, the above relation takes the form

$$
\begin{aligned}
\frac{1}{\kappa_{4}^{2}} & =\frac{f_{0}}{k}+\frac{2 \Phi_{0}^{2} e^{2 k y_{0}}}{k^{\lambda}}(2 k)^{\lambda-1} \int_{2 k y_{0}}^{\infty} d t t^{-\lambda} e^{-t} \\
& =\frac{f_{0}}{k}+\frac{2^{\lambda} \Phi_{0}^{2} e^{2 k y_{0}}}{k} \Gamma\left(1-\lambda, 2 k y_{0}\right) .
\end{aligned}
$$

Above, we have used the upper incomplete gamma function $\Gamma(s, x)$, defined as

$$
\Gamma(s, x) \equiv \int_{x}^{\infty} d t t^{s-1} e^{-t} .
$$

The properties of the incomplete gamma function as well as the expressions giving its numerical values are discussed in Appendix B. With the use of Eq. (5.22) and the relation $1 / \kappa_{4}^{2}=M_{P l}^{2} /(8 \pi)$, we finally obtain

$$
M_{P l}^{2}=\frac{8 \pi \Phi_{0}^{2}}{k}\left\{\frac{f_{0}}{\Phi_{0}^{2}}+2^{\lambda} e^{2 k y_{0}} \Gamma\left(1-\lambda, 2 k y_{0}\right)\right\} .
$$

Demanding the positivity of the effective four-dimensional gravitational scale $M_{P l}^{2}$, we are led to the additional constraint

$$
\frac{f_{0}}{\Phi_{0}^{2}}>-2^{\lambda} e^{2 k y_{0}} \Gamma\left(1-\lambda, 2 k y_{0}\right)
$$

Finally, substituting the total energy density on the brane from Eq. (5.18) and the expressions of the functions $f(y)$, $\Phi(y)^{6}$ and $V_{B}(y)$ in Eq. (3.21), we can verify that the effective four-dimensional cosmological constant on the brane is zero, as expected.

\section{Energy conditions and the parameter space}

In this subsection, we will study the parameter space of the ratio $f_{0} / \Phi_{0}^{2}$ and the dimensionless parameter $k y_{0}$. The value of the parameter $\lambda$ may be also varied; however, once fixed, it determines the allowed values of the parameter $k y_{0}$ through the constraint (5.3). As usual, we will investigate the parameter regimes where the inequalities (5.15), (5.19), and (5.25) are satisfied.

In Fig. 12, we depict the aforementioned parameter space for the value $\lambda=3$. We also depict the curves of the

\footnotetext{
${ }^{6}$ For the calculation of the effective four-dimensional cosmological constant on the brane $\Lambda_{4}$, it is more convenient to use the relation (5.2) instead of the explicit form of the scalar field $\Phi(y)$ as given by Eq. (5.6).
}

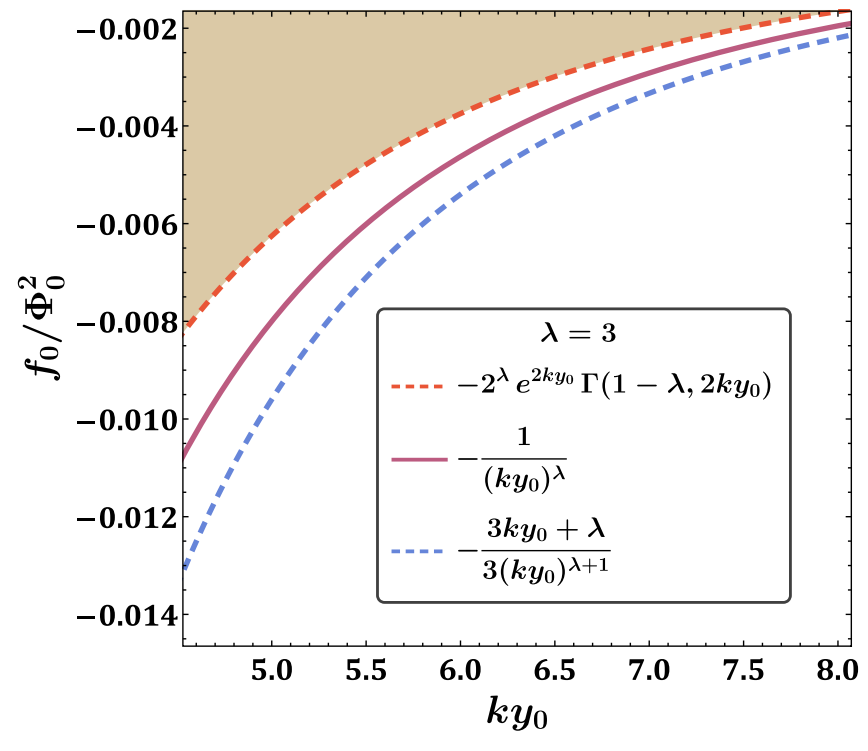

FIG. 12. Parameter space between the ratio $f_{0} / \Phi_{0}^{2}$ and the parameter $k y_{0}$, for $\lambda=3$. The figure depicts also the plots of the expressions appearing on the right-hand sides of the inequalities (5.15), (5.19), and (5.25).

expressions on the right-hand sides of the inequalities (5.15), (5.19), and (5.25). Although the corresponding curves have been drawn for a particular value of $\lambda$, it turns out that their relative position remains the same for any allowed value of the parameters $\lambda$ and $k y_{0}$; namely we always have

$$
-2^{\lambda} e^{2 k y_{0}} \Gamma\left(1-\lambda, 2 k y_{0}\right)>-\frac{1}{\left(k y_{0}\right)^{\lambda}}>-\frac{3 k y_{0}+\lambda}{3\left(k y_{0}\right)^{\lambda+1}} .
$$

Clearly, this means that only the inequalities (5.19) and (5.25) can be simultaneously satisfied. Therefore, we may easily obtain a model with a positive four-dimensional gravitational constant and a positive total energy density on the brane. However, in that case, we will not be able to satisfy the weak energy conditions by the bulk matter close and on the brane. This means that the energy density $\rho$ will be negative at the location of the brane with the pressure having the exact opposite value.

\section{A LINEAR-EXPONENTIAL COUPLING FUNCTION IN TERMS OF $y$}

In this case, we consider the following coupling function $f(y)$ in terms of the coordinate $y$ :

$$
f(y)=f_{0}+f_{1} k y e^{-\lambda k y} .
$$

We also assume that $f_{1} \in \mathbb{R} \backslash\{0\}$ and $\lambda \in(0,+\infty)$ in order for $f(y)$ to satisfy the physical constraints discussed at the end of Sec. II.

Let us start by deriving first the bulk solution. Substituting the aforementioned coupling function in Eq. (2.18), we obtain 


$$
\left[\Phi^{\prime}(y)\right]^{2}=f_{1} k^{2} e^{-\lambda k y}[2 \lambda-1-\lambda(\lambda-1) k y] .
$$

Since the scalar field $\Phi(y)$ should be a real-valued function, it is obvious that $\left[\Phi^{\prime}(y)\right]^{2} \geq 0$ for all values of $y$ which are greater or equal to zero. Let us first assume that $f_{1}<0$; then, demanding that $\left[\Phi^{\prime}(0)\right]^{2} \geq 0$, we obtain the constraint $\lambda \leq 1 / 2$. On the other hand, for large values of the $y$ coordinate (i.e., at $y=y_{0} \gg 1$ ), demanding that $\left[\Phi^{\prime}\left(y_{0}\right)\right]^{2} \geq 0$ leads to $\lambda \geq 1$. $^{7}$ However, these two constraints are incompatible, which leads us to deduce that the parameter $f_{1}$ should be strictly positive. In that case, a similar argument as above leads to the allowed regime $\lambda \in\left[\frac{1}{2}, 1\right]$. Moreover, since $f_{1}$ is positive, we may set $f_{1}=\Phi_{0}^{2}$, and assume for simplicity that $\Phi_{0} \in(0,+\infty)$.

For $\lambda=1$, we can easily integrate Eq. (6.2) with respect to $y$, and determine the expression of the function of the scalar field $\Phi(y)$. Then, we obtain

$$
\Phi_{ \pm}(y)= \pm 2 \Phi_{0} e^{-k y / 2} .
$$

Above, we have used again the translational symmetry of the gravitational field equations with respect to the value of the scalar field in order to eliminate an additive integration constant. By inverting the above function, we can express the coupling function in terms of the scalar field $\Phi$, namely

$$
f(\Phi)=f_{0}-\frac{\Phi^{2}}{2} \ln \left(\frac{\Phi}{2 \Phi_{0}}\right) .
$$

Equation (6.2) is more difficult to solve in the remaining $\lambda$-parameter regime, i.e., for $\lambda \in\left[\frac{1}{2}, 1\right)$. In that case, Eq. (6.2) leads to

$$
\begin{aligned}
\Phi_{ \pm}(y)= & \pm \Phi_{0} k \int d y e^{-\lambda k y / 2} \sqrt{2 \lambda-1-\lambda(\lambda-1) k y} \\
= & \pm \frac{2 \Phi_{0}}{\lambda}\left[-e^{-\lambda k y / 2} \sqrt{2 \lambda-1-\lambda(\lambda-1) k y}\right. \\
& \left.+\int d y e^{-\lambda k y / 2} \frac{d}{d y}(\sqrt{2 \lambda-1-\lambda(\lambda-1) k y})\right] .
\end{aligned}
$$

Focusing on the second term of the right-hand side of the above relation, and due to the fact that $\lambda \in\left[\frac{1}{2}, 1\right)$, we can write

$$
\begin{aligned}
& \int d y e^{-\frac{\lambda k y}{2}} \frac{d}{d y}(\sqrt{2 \lambda-1-\lambda(\lambda-1) k y}) \\
& =e^{\frac{2 \lambda-1}{2(1-\lambda)}} \sqrt{2(1-\lambda)} \int d y e^{-\frac{2 \lambda-1-\lambda(\lambda-1) k y}{2(1-\lambda)}} \frac{d}{d y} \sqrt{\frac{2 \lambda-1-\lambda(\lambda-1) k y}{2(1-\lambda)}} \\
& =\sqrt{\frac{\pi(1-\lambda)}{2}} e^{\frac{2 \lambda-1}{2(1-\lambda)}} \operatorname{erf}\left(\sqrt{\frac{2 \lambda-1-\lambda(\lambda-1) k y}{2(1-\lambda)}}\right)
\end{aligned}
$$

\footnotetext{
${ }^{7}$ Here, we have used the fact that, for large values of $y$, only the term proportional to $k y$ mainly contributes to the value of $\left[\Phi^{\prime}(y)\right]^{2}$.
}

where we have used the error function, defined as

$$
\operatorname{erf}(x)=\frac{2}{\sqrt{\pi}} \int_{0}^{x} d t e^{-t^{2}}
$$

and its property

$$
\frac{d}{d x} \operatorname{erf}(g(x))=\frac{2}{\sqrt{\pi}} e^{-g(x)^{2}} \frac{d g(x)}{d x} .
$$

Combining Eqs. (6.5) and (6.6) we obtain

$$
\begin{aligned}
\Phi_{ \pm}(y)= & \pm \frac{2 \Phi_{0}}{\lambda}\left[-e^{-\lambda k y / 2} \sqrt{2 \lambda-1-\lambda(\lambda-1) k y}\right. \\
& \left.+\sqrt{\frac{\pi(1-\lambda)}{2}} e^{\frac{2 \lambda-1}{2(1-\lambda)}} \operatorname{erf}\left(\sqrt{\frac{2 \lambda-1-\lambda(\lambda-1) k y}{2(1-\lambda)}}\right)\right] .
\end{aligned}
$$

In this case, it is not possible to invert the function $\Phi(y)$ in order to find the form of the coupling function $f(\Phi)$. However, from Eq. (6.2) and for $\lambda \in\left[\frac{1}{2}, 1\right)$, it is straightforward to deduce that $\Phi^{\prime}(y) \neq 0$ for all $y>0$; this, again, means that $\Phi(y)$ does not have any extremum, and is therefore a one-to-one function. This property will be of use in the evaluation of the junction conditions on the brane.

In Figs. 13(a) and 13(b), we present the warp factor, the coupling function, and the scalar field for particular choices of values for the parameters of the model. The coupling function $f(y)$ adopts the same constant value $f_{0}$ at the location of our brane and at asymptotic infinity while reaching a maximum value at some intermediate distance off our brane, as depicted in Fig. 13(a). In Fig. 13(b), the scalar field presents two distinct profiles, for $\lambda=1$ and $\lambda \in\left[\frac{1}{2}, 1\right)$ due to the two different solutions given by Eqs. (6.3) and (6.7), respectively. In all cases, though, $\Phi_{ \pm}(y)$ remains everywhere finite approaching a constant value at asymptotic infinity: for $\lambda=1$ this constant is zero, while for $\lambda \in\left[\frac{1}{2}, 1\right)$ this is given by the expression

$\lim _{y \rightarrow \pm \infty} \Phi_{ \pm}(y)= \pm \frac{2 \Phi_{0}}{\lambda} \sqrt{\frac{\pi(1-\lambda)}{2}} e^{\frac{2 \lambda-1}{2(1-\lambda)}}, \quad \lambda \in\left[\frac{1}{2}, 1\right)$.

In the above, we have used the fact that the limit of the error function appearing in Eq. (6.7), as $y \rightarrow+\infty$, is unity. Due to the $\mathbf{Z}_{2}$ symmetry imposed on our model, the same limit will hold for the scalar field also for $y \rightarrow-\infty$.

From Eq. (2.19), we may now determine the potential of the scalar field $V_{B}(y)$ in the bulk by using the expression of the coupling function $f(y)$. Then, we find 


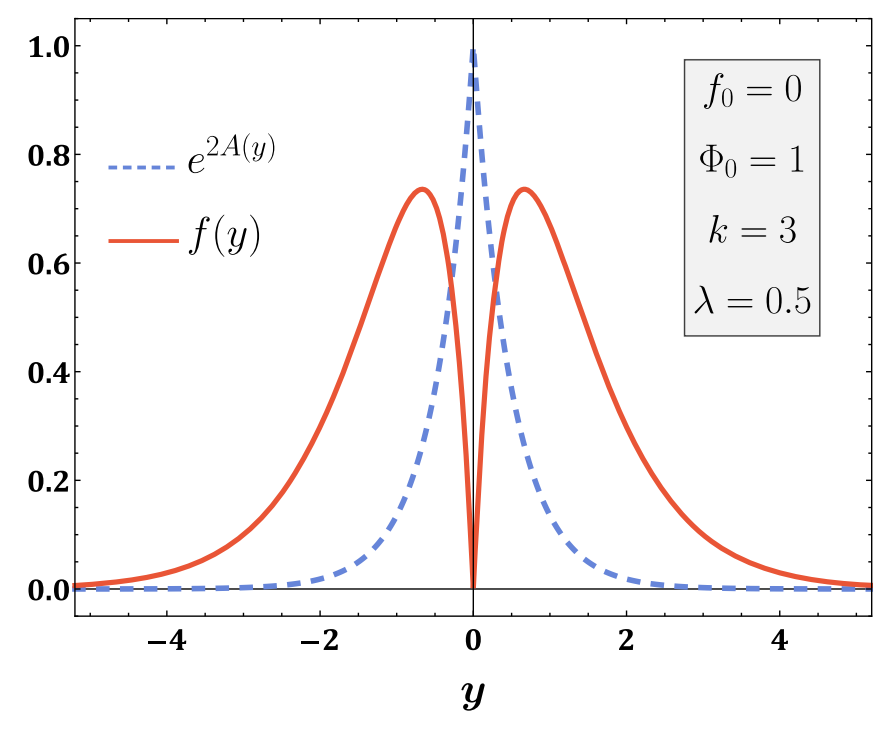

(a)

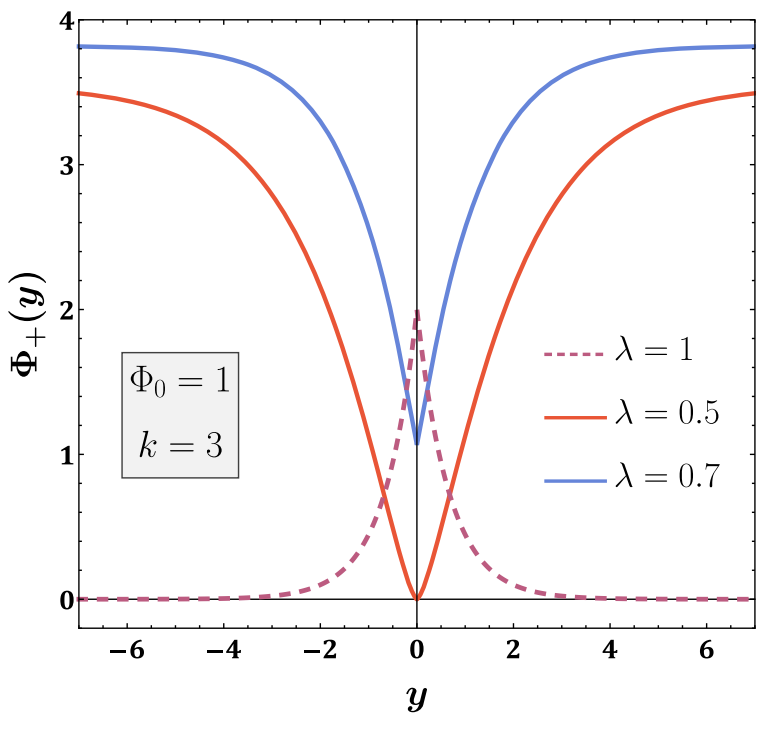

(b)

FIG. 13. (a) Coupling function $f(y)$ and the warp factor $e^{-2 A(y)}$ in terms of the $y$ coordinate for $f_{0}=0, \Phi_{0}=1, k=3$, and $\lambda=0.5$. (b) Scalar field $\Phi_{+}(y)$ for $\Phi_{0}=1, k=3$, and $\lambda=1,0.5,0.7$.

$$
\begin{aligned}
V_{B}(y) & =-\Lambda_{5}-6 k^{2} f_{0}+\Phi_{0}^{2} k^{2} e^{-\lambda k y}\left[\frac{7}{2}+\lambda-k y\left(\frac{\lambda^{2}}{2}+\frac{7 \lambda}{2}+6\right)\right], \\
\lambda & \in\left[\frac{1}{2}, 1\right] .
\end{aligned}
$$

On the other hand, the energy density $\rho(y)$ and pressure $p(y)=p^{i}(y)=p^{y}(y)$ may be computed by employing Eqs. (3.6) and (3.7); then, we obtain

$$
\begin{aligned}
\rho(y) & =-p(y)=-6 k^{2} f(y) \\
& =-6 k^{2}\left(f_{0}+\Phi_{0}^{2} k y e^{-\lambda k y}\right) .
\end{aligned}
$$

In order to satisfy the weak energy conditions close and on the brane, we should have again $\rho(0) \geq 0$, or equivalently $f(0) \leq 0$; hence, we are led to the following inequality:

$$
\frac{f_{0}}{\Phi_{0}^{2}} \leq 0 .
$$

In Fig. 14, we present the energy density and pressure as well as the profile of the bulk potential for the same values of parameters as in Fig. 13 for easy comparison. We observe that both components and the bulk potential are everywhere finite, reach their maximum values at a finite distance from our brane, and reduce to a constant value (which here is taken to be zero) at large distances.

Let us now turn to the junction conditions introduced in the theory at the location of the brane. From Eqs. (5.16) and (5.17), we obtain in a similar way the conditions

$$
\begin{gathered}
\sigma+\left.V_{b}(\Phi)\right|_{y=0}=2 k\left(3 f_{0}-\Phi_{0}^{2}\right), \\
\left.\partial_{y} V_{b}\right|_{y=0}=8 k^{2} \Phi_{0}^{2}\left(\lambda^{2}-\lambda+\frac{5}{4}\right),
\end{gathered}
$$

for $\lambda \in\left[\frac{1}{2}, 1\right]$. The total energy density on the brane will be positive if and only if $\sigma+\left.V_{b}(\Phi)\right|_{y=0}>0$, which results to

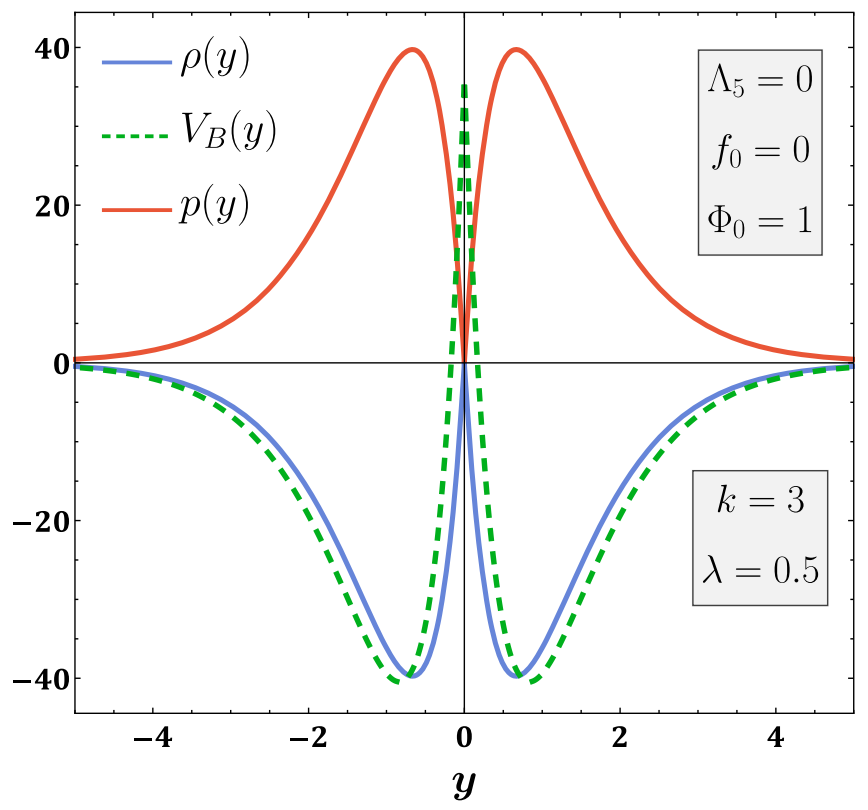

FIG. 14. Energy density $\rho$ and pressure $p$ of the system together with the scalar potential $V_{B}$ in terms of the coordinate $y$ for $\Lambda_{5}=0, f_{0}=0, \Phi_{0}=1, k=3$, and $\lambda=0.5$. 


$$
\frac{f_{0}}{\Phi_{0}^{2}}>\frac{1}{3} .
$$

Next, we are going to evaluate the effective four-dimensional gravitational constant on the brane. Using Eq. (3.17), we obtain

$$
\frac{1}{\kappa_{4}^{2}}=\frac{M_{P l}^{2}}{8 \pi}=\frac{\Phi_{0}^{2}}{k}\left[\frac{f_{0}}{\Phi_{0}^{2}}+\frac{2}{(2+\lambda)^{2}}\right] .
$$

For a robust effective theory on the brane, it is imperative to have a positive four-dimensional gravitational constant; thus, we must satisfy the following constraint:

$$
\frac{f_{0}}{\Phi_{0}^{2}}>-\frac{2}{(2+\lambda)^{2}} .
$$

Once again, as expected, the effective four-dimensional cosmological constant on the brane may be found to be zero with the use of Eq. (3.21).

It is straightforward to study whether the inequalities (6.11), (6.14), and (6.16) can be simultaneously satisfied. By merely observing the first two of them, it is easy to deduce that they are incompatible since the value of $f_{0} / \Phi_{0}^{2}$ can be either positive or negative. Additionally, as we already mentioned, the parameter $\lambda$ takes values in the range $\left[\frac{1}{2}, 1\right]$. In this case, it holds that

$$
-\frac{8}{25} \leq-\frac{2}{(2+\lambda)^{2}} \leq-\frac{2}{9}
$$

Hence, we can simultaneously satisfy either the inequalities (6.11) and (6.16), or (6.14) and (6.16). In particular, a positive four-dimensional gravitational scale $M_{P l}^{2}$ can be combined with the bulk matter satisfying the weak energy conditions close to the brane, for

$$
-\frac{2}{9} \leq \frac{f_{0}}{\Phi_{0}^{2}} \leq 0
$$

or with a positive total energy density on the brane, for

$$
\frac{f_{0}}{\Phi_{0}^{2}}>\frac{1}{3} .
$$

The particular solution depicted in Fig. 14 corresponds to the value $f_{0}=0$; therefore, it is characterized by a negative energy density inside the bulk, which violates the energy conditions. Note, however, that at the location of our brane, both the energy density and pressure are zero while the bulk potential is positive.

\section{A DOUBLE-EXPONENTIAL SCALAR FIELD IN TERMS OF $y$}

In this section, we follow an alternative approach and consider the following expression for the scalar field in terms of the coordinate $y$ :

$$
\Phi(y)=\Phi_{0} e^{-\mu^{2} e^{k y}} .
$$

Although this expression seems similar to the subcase of the quadratic coupling function with $\lambda=-1 / 4$, it differs significantly as it will become clear from the expressions of the coupling function $f(\Phi)$ and the scalar potential $V_{B}(\Phi)$. Moreover, it is obvious that both parameters $\Phi_{0}$ and $\mu$ can now take values in the entire set of real numbers except zero. With the form of the scalar field already known, it is straightforward to derive the corresponding forms of the coupling function, bulk potential, and components of the energy-momentum tensor. Starting with the coupling function, upon substituting the aforementioned expression of the scalar field in Eq. (2.18), we readily obtain

$$
f(y)=f_{0}-f_{1} e^{-k y}-\frac{\Phi_{0}^{2}}{4 \mu^{2}} e^{-2 \mu^{2} e^{k y}}\left(\mu^{2}+e^{-k y}\right) .
$$

In the above result, the parameter $f_{1}$ is allowed to take values in the whole set of real numbers, while the allowed values for the parameter $f_{0}$ will be examined shortly. Inverting the function $\Phi(y)$, the expression of the coupling function in terms of the scalar field reads

$$
f(\Phi)=f_{0}+\frac{f_{1} \mu^{2}}{\ln \left(\Phi / \Phi_{0}\right)}-\frac{\Phi^{2}}{4}\left(1-\frac{1}{\ln \left(\Phi / \Phi_{0}\right)}\right) .
$$

The scalar potential $V_{B}(y)$ can then be evaluated employing Eqs. (2.19) and (7.2). Then, we find

$$
\begin{aligned}
V_{B}(y)= & -\Lambda_{5}-6 k^{2} f_{0}+10 k^{2} f_{1} e^{-k y} \\
& +\frac{\Phi_{0}^{2} k^{2}}{2 \mu^{2}} e^{-2 \mu^{2} e^{k y}}\left(5 e^{-k y}+7 \mu^{2}+4 \mu^{4} e^{k y}+\mu^{6} e^{2 k y}\right),
\end{aligned}
$$

in terms of the $y$ coordinate, or

$$
\begin{aligned}
V_{B}(\Phi)= & -\Lambda_{5}-6 k^{2} f_{0}-\frac{10 k^{2} f_{1} \mu^{2}}{\ln \left(\Phi / \Phi_{0}\right)}+\frac{\Phi_{0}^{2} k^{2}}{2}\left\{-\frac{5}{\ln \left(\Phi / \Phi_{0}\right)}\right. \\
& \left.+7-4 \ln \left(\frac{\Phi}{\Phi_{0}}\right)+\left[\ln \left(\frac{\Phi}{\Phi_{0}}\right)\right]^{2}\right\},
\end{aligned}
$$

in terms of the scalar field. In Figs. 15(a) and 15(b), we display the profiles of the coupling function and scalar potential in terms of the $y$ coordinate, for particular values of the parameters of the model. The varying parameter here is $f_{1}$, which is clearly the decisive one for the form of both functions. We observe that for positive $f_{1}$, the coupling function takes its lowest value at the location of the brane 


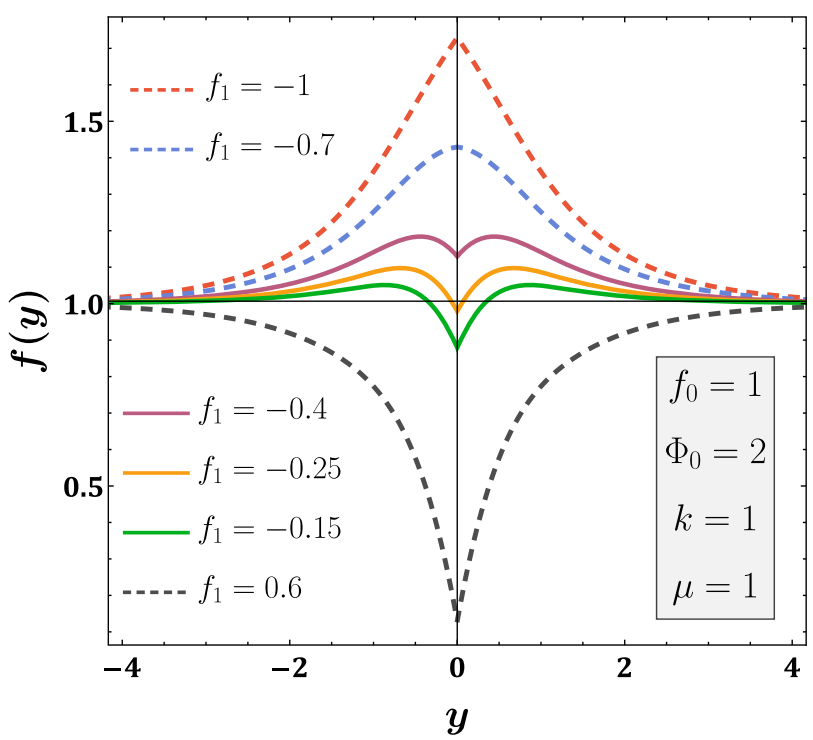

(a)

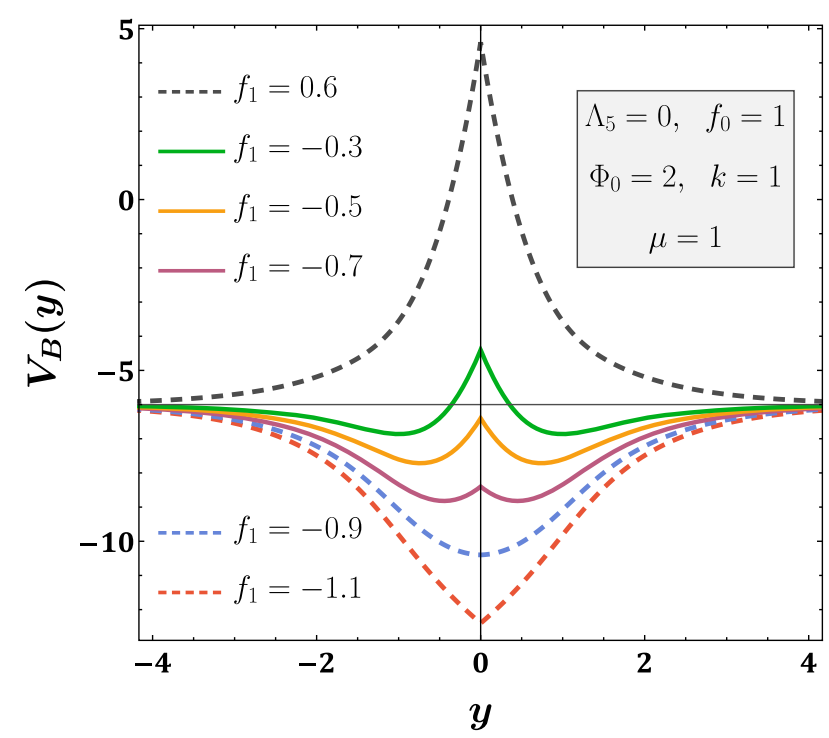

(b)

FIG. 15. (a) Coupling function in terms of the $y$ coordinate for $f_{0}=1, \quad \Phi_{0}=2, k=1, \quad \mu=1, \quad$ and $f_{1}=$ $-1,-0.7,-0.4,-0.25,-0.15,0.6$ (from top to bottom). (b) Scalar potential $V_{B}$ in terms of the coordinate $y$ for $\Lambda_{5}=0, f_{0}=1$, $\Phi_{0}=2, k=1, \mu=1$, and $f_{1}=-1.1,-0.9,-0.7,-0.5,-0.3,0.6$ (from bottom to top).

while, as $f_{1}$ gradually takes larger negative values, the coupling function eventually exhibits a peak at the location of the brane. The bulk potential has almost the exact opposite profile of the coupling function: it acquires a maximum, positive value at the location of our brane for $f_{1}>0$ while it turns to globally negative values for $f_{1}<0$. For every set of values of the parameters, though, both functions are everywhere finite and reduce to a constant value at large distances -this value, when $\Lambda_{5}=0$, is determined by $f_{0}$.

Finally, the energy density $\rho(y)$ and pressure $p(y)=$ $p^{i}(y)=p^{y}(y)$ components may be computed as usual by employing Eqs. (3.6) and (3.7), in which case we are led to the results

$$
\begin{aligned}
\rho(y) & =-p(y)=-6 k^{2} f(y) \\
& =-6 k^{2}\left[f_{0}-f_{1} e^{-k y}-\frac{\Phi_{0}^{2}}{4 \mu^{2}} e^{-2 \mu^{2} e^{k y}}\left(\mu^{2}+e^{-k y}\right)\right] .
\end{aligned}
$$

In order to satisfy the weak energy conditions close and on the brane, we demand again that $f(0)<0$; hence, we obtain the following inequality:

$$
\frac{f_{0}}{\Phi_{0}^{2}}<\frac{f_{1}}{\Phi_{0}^{2}}+\frac{\mu^{2}+1}{4 \mu^{2}} e^{-2 \mu^{2}}
$$

Turning now to the junction conditions, from Eqs. (5.16), (5.17) and using also the relations (7.1)-(7.3), we obtain $\sigma+\left.V_{b}(\Phi)\right|_{y=0}=6 k f_{0}-8 k f_{1}-\frac{\Phi_{0}^{2} k e^{-2 \mu^{2}}}{2 \mu^{2}}\left(4+5 \mu^{2}+2 \mu^{4}\right)$,

$$
\left.\partial_{y} V_{b}\right|_{y=0}=8 k^{2} f_{1}+\frac{2 \Phi_{0}^{2} k^{2}}{\mu^{2}} e^{-2 \mu^{2}}\left(1+2 \mu^{2}+2 \mu^{4}+\mu^{6}\right) .
$$

In the second of the above equations, we have used the relation $\partial_{y} V_{b}=\Phi^{\prime} \partial_{\Phi} V_{b}$. In order to have a positive total energy density on the brane, we should have

$$
\frac{f_{0}}{\Phi_{0}^{2}}>\frac{4}{3} \frac{f_{1}}{\Phi_{0}^{2}}+\frac{e^{-2 \mu^{2}}}{12 \mu^{2}}\left(4+5 \mu^{2}+2 \mu^{4}\right) .
$$

In the context of the effective theory on the brane, we may evaluate the four-dimensional gravitational scale using Eqs. (3.17) and (7.2). Then,

$$
\begin{aligned}
\frac{1}{\kappa_{4}^{2}} & =\frac{f_{0}}{k}-\frac{2 f_{1}}{3 k}-\frac{2 \Phi_{0}^{2}}{4 \mu^{2}} \int_{0}^{\infty} d y e^{-2 \mu^{2} e^{k y}-2 k y}\left(\mu^{2}+e^{-k y}\right) \\
& =\frac{f_{0}}{k}-\frac{2 f_{1}}{3 k}-\frac{2 \Phi_{0}^{2} \mu^{2}}{k}\left(\frac{e^{-2 \mu^{2}}}{8 \mu^{4}}-\mu^{2} \int_{2 \mu^{2}}^{\infty} d t t^{-4} e^{-t}\right)
\end{aligned}
$$

where, in the second line, we first set $e^{-k y}=w$ and then $\frac{2 \mu^{2}}{w}=t$. The integral in the above expression is the upper incomplete gamma function $\Gamma\left(-3,2 \mu^{2}\right)$ as one may easily conclude from Eq. (B1). The latter quantity, through Eq. (B4), may be written as

$$
\Gamma\left(-3,2 \mu^{2}\right)=\frac{1}{6}\left[\frac{e^{-2 \mu^{2}}}{4 \mu^{6}}\left(1-\mu^{2}+2 \mu^{4}\right)-\Gamma\left(0,2 \mu^{2}\right)\right],
$$

where 


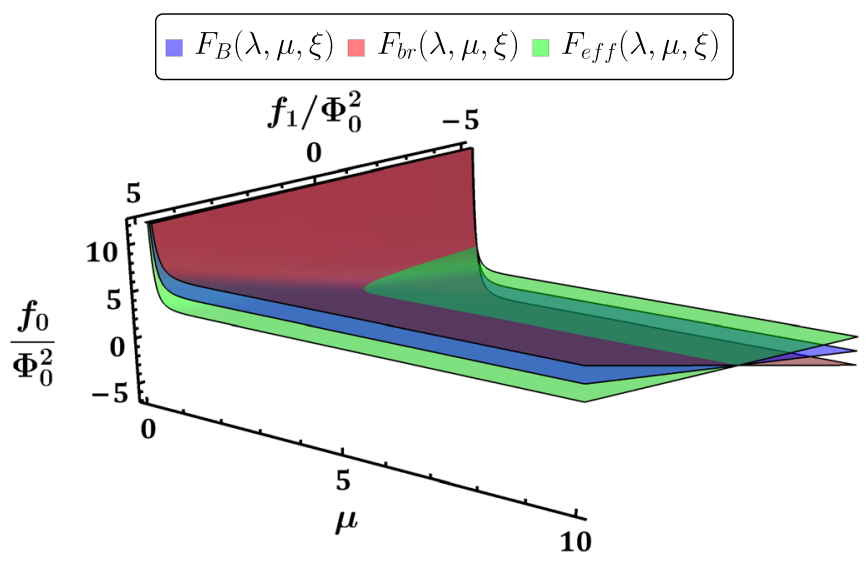

(a)

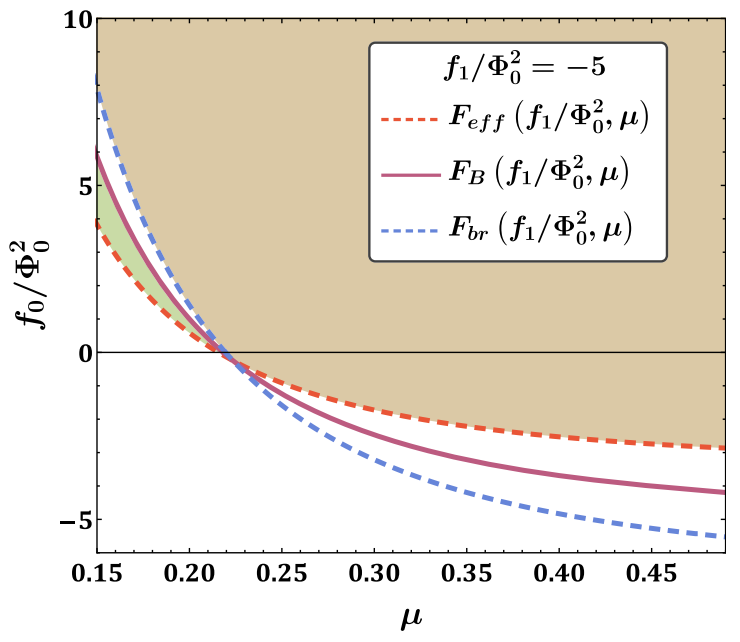

(b)

FIG. 16. (a) 3D parameter space of the quantities $f_{0} / \Phi_{0}^{2}, f_{1} / \Phi_{0}^{2}$, and $\mu$. (b) $2 \mathrm{D}$ parameter space of the quantities $f_{0} / \Phi_{0}^{2}$ and $\mu$ for $f_{1} / \Phi_{0}^{2}=-5$. The figures also present the corresponding surfaces or curves of $F_{B}\left(f_{1} / \Phi_{0}^{2}, \mu\right), F_{b r}\left(f_{1} / \Phi_{0}^{2}, \mu\right)$, and $F_{\text {eff }}\left(f_{1} / \Phi_{0}^{2}, \mu\right)$.

$$
\Gamma\left(0,2 \mu^{2}\right)=-\gamma-\ln \left(2 \mu^{2}\right)-\sum_{m=1}^{\infty} \frac{(-1)^{m} 2^{m} \mu^{2 m}}{m(m !)}
$$

Hence, we finally obtain

$$
\begin{aligned}
\frac{1}{\kappa_{4}^{2}}= & \frac{M_{P l}^{2}}{8 \pi}=\frac{f_{0}}{k}-\frac{2 f_{1}}{3 k}-\frac{\Phi_{0}^{2} e^{-2 \mu^{2}}}{12 k \mu^{2}}\left(2+\mu^{2}-2 \mu^{4}\right) \\
& -\frac{\Phi_{0}^{2} \mu^{4}}{3 k} \Gamma\left(0,2 \mu^{2}\right) .
\end{aligned}
$$

Demanding as usual a positive four-dimensional gravitational constant, we find that the following inequality must be satisfied:

$$
\frac{f_{0}}{\Phi_{0}^{2}}>\frac{2}{3} \frac{f_{1}}{\Phi_{0}^{2}}+\frac{e^{-2 \mu^{2}}}{12 \mu^{2}}\left(2+\mu^{2}-2 \mu^{4}\right)+\frac{\mu^{4}}{3} \Gamma\left(0,2 \mu^{2}\right) .
$$

As before, the evaluation of the effective four-dimensional cosmological constant gives $\Lambda_{4}=0$.

Finally, we investigate the parameter space of the quantities $f_{0} / \Phi_{0}^{2}, f_{1} / \Phi_{0}^{2}$, and $\mu$, in an attempt to simultaneously satisfy the inequalities (7.7), (7.10), and (7.15). In Fig. 16(a), we depict the aforementioned parameter space as well as the surfaces which correspond to the right-hand sides of these inequalities. We observe that there is no point in the parameter space at which all three inequalities can be satisfied. It is possible though to satisfy two out of these three inequalities simultaneously; which two are satisfied depends on the values of the parameters. For $f_{1} / \Phi_{0}^{2}=5$, for example, the situation is simple as the relative position of the three surfaces remains the same independently of the value of $\mu$ : thus, we may have a positive effective cosmological constant and a positive total energy density on the brane for low values of $f_{0} / \Phi_{0}^{2}$ whereas for large values of $f_{0} / \Phi_{0}^{2}$ we have a positive $M_{P l}^{2}$ and the weak energy conditions are satisfied close to our brane. For $f_{1} / \Phi_{0}^{2}=-5$, the situation changes and the pair of conditions satisfied depends on the values of all three parameters - the exact situation is depicted in Fig. 16(b) where the green region corresponds to the area where the inequalities (7.7) and (7.15) are satisfied, and the brown region to the area where (7.10) and (7.15) are satisfied.

In Fig. 17, we present the graphs of the energy density $\rho(y)$ and pressure $p(y)$ as well as the potential of the scalar

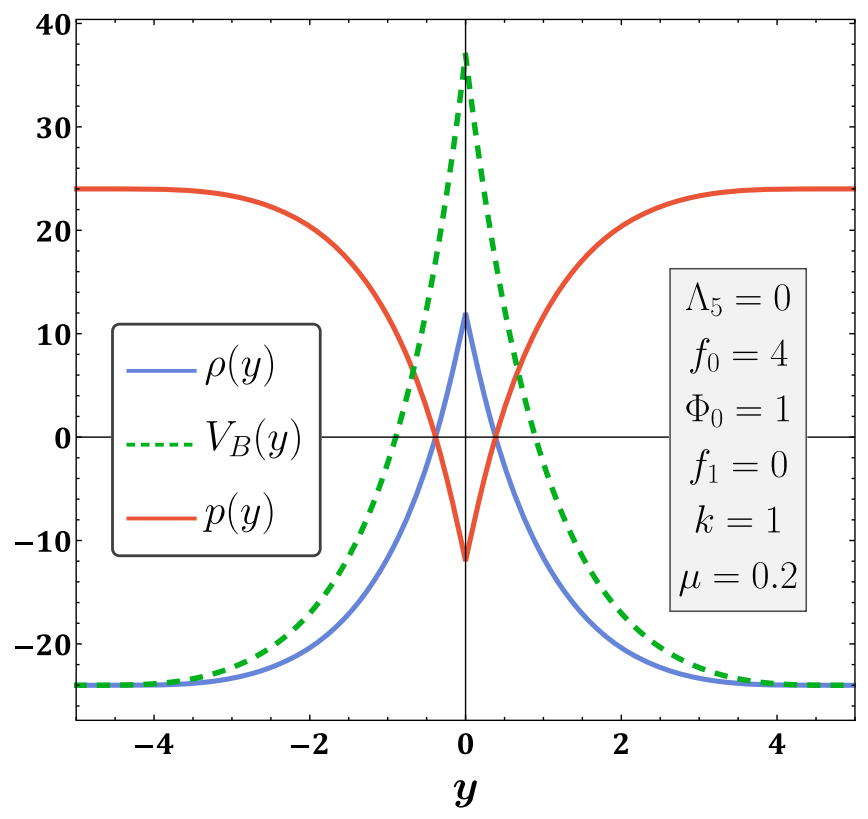

FIG. 17. Energy density $\rho$ and pressure $p$ of the system together with the scalar potential $V_{B}$ in terms of the coordinate $y$ for $\Lambda_{5}=0, f_{0}=4, \Phi_{0}=1, f_{1}=0, k=1$, and $\mu=0.2$. 
field $V_{B}(y)$ in terms of the $y$ coordinate for $\Lambda_{5}=0, f_{0}=4$, $\Phi_{0}=1, f_{1}=0, k=1$, and $\mu=0.2$. As we can see in the figure, the values of the parameters are appropriately chosen to satisfy the weak energy conditions close to the brane.

\section{A HYPERBOLIC-TANGENT SCALAR FIELD IN TERMS OF $y$}

Following the same line of thinking as in the previous section, we now consider the following expression for the scalar field in terms of the coordinate $y$ :

$$
\Phi(y)=\Phi_{0} \tanh (k y),
$$

where $\Phi_{0} \in \mathbb{R} \backslash\{0\}$. Substituting the above expression of the scalar field in Eq. (2.18), we obtain the form of the coupling function

$f(y)=f_{0}-f_{1} e^{-k y}+\Phi_{0}^{2} e^{-k y} \arctan \left(e^{k y}\right)-\frac{\Phi_{0}^{2} e^{2 k y}\left(e^{2 k y}-1\right)}{3\left(e^{2 k y}+1\right)^{2}}$.

Again, the parameter $f_{1}$ is allowed to take values in the whole set of real numbers, while the allowed values for the parameter $f_{0}$ will be examined shortly. By inverting the function $\Phi(y)$, we may express the coupling function in terms of the scalar field to get

$$
\begin{aligned}
f(\Phi)= & f_{0}-f_{1} \sqrt{\frac{\Phi_{0}-\Phi}{\Phi_{0}+\Phi}} \\
& +\Phi_{0}^{2} \sqrt{\frac{\Phi_{0}-\Phi}{\Phi_{0}+\Phi}} \arctan \left(\sqrt{\frac{\Phi_{0}+\Phi}{\Phi_{0}-\Phi}}\right)-\frac{\Phi\left(\Phi+\Phi_{0}\right)}{6} .
\end{aligned}
$$

Similarly, the scalar potential $V_{B}(y)$ can be evaluated from Eq. (2.19) with the use of (8.2); then, we find

$$
\begin{aligned}
V_{B}(y)= & -\Lambda_{5}-6 k^{2} f_{0}+10 k^{2} e^{-k y}\left[f_{1}-\Phi_{0}^{2} \arctan \left(e^{k y}\right)\right] \\
& +\frac{2 k^{2} \Phi_{0}^{2}}{3} \frac{6+19 e^{2 k y}+19 e^{4 k y}-3 e^{6 k y}+3 e^{8 k y}}{\left(e^{2 k y}+1\right)^{4}} .
\end{aligned}
$$

In terms of the scalar field, the scalar potential is alternatively written as

$$
\begin{aligned}
V_{B}(\Phi)= & -\Lambda_{5}-6 k^{2} f_{0} \\
& +10 k^{2} \sqrt{\frac{\Phi_{0}-\Phi}{\Phi_{0}+\Phi}}\left[f_{1}-\Phi_{0}^{2} \arctan \left(\sqrt{\frac{\Phi_{0}+\Phi}{\Phi_{0}-\Phi}}\right)\right] \\
& +\frac{k^{2}}{6 \Phi_{0}^{2}}\left(3 \Phi^{4}+8 \Phi^{3} \Phi_{0}+4 \Phi^{2} \Phi_{0}^{2}-14 \Phi \Phi_{0}^{3}+11 \Phi_{0}^{4}\right) .
\end{aligned}
$$

The profiles of the coupling function and scalar potential in this case are qualitatively the same as the ones in the double-exponential case of the previous section depicted in Figs. 15(a) and 15(b). Again, as the parameter $f_{1}$ changes from positive to negative values, the coupling function acquires an increasingly larger positive value at the location of our brane; with the same variation, the scalar potential changes from globally positive-definite to globally negative-definite values. As before, both functions remain finite everywhere in the bulk and adopt constant values at large distances.

The energy density $\rho(y)$ and pressure $p(y)=p^{i}(y)=$ $p^{y}(y)$ may be computed by employing Eqs. (3.6) and (3.7), and we are led to the result

$$
\begin{aligned}
\rho(y)= & -p(y)=-6 k^{2} f(y) \\
= & -6 k^{2}\left[f_{0}-f_{1} e^{-k y}+\Phi_{0}^{2} e^{-k y} \arctan \left(e^{k y}\right)\right. \\
& \left.-\frac{\Phi_{0}^{2} e^{2 k y}\left(e^{2 k y}-1\right)}{3\left(e^{2 k y}+1\right)^{2}}\right] .
\end{aligned}
$$

In order to satisfy the weak energy conditions close and on the brane, we impose the condition that $\rho(0)>0$, or $f(0)<0$; hence, we obtain the following inequality:

$$
\frac{f_{0}}{\Phi_{0}^{2}}<\frac{f_{1}}{\Phi_{0}^{2}}-\frac{\pi}{4}
$$

The junction conditions (5.16), (5.17), employing the relations (8.1)-(8.3), now yield

$$
\begin{gathered}
\sigma+\left.V_{b}(\Phi)\right|_{y=0}=6 k f_{0}-8 k f_{1}+2 k \Phi_{0}^{2}\left(\pi-\frac{1}{3}\right), \\
\left.\partial_{y} V_{b}\right|_{y=0}=8 k^{2} f_{1}+2 k^{2} \Phi_{0}^{2}\left(\frac{7}{3}-\pi\right) .
\end{gathered}
$$

Therefore, in order to have a positive total energy density on the brane, we demand the condition

$$
\frac{f_{0}}{\Phi_{0}^{2}}>\frac{4}{3} \frac{f_{1}}{\Phi_{0}^{2}}+\frac{1}{9}-\frac{\pi}{3}
$$

Let us also evaluate the effective four-dimensional gravitational constant on the brane. Using Eq. (3.17), we obtain

$$
\begin{aligned}
\frac{1}{\kappa_{4}^{2}}= & 2 \int_{0}^{\infty} d y e^{-2 k y}\left[f_{0}-f_{1} e^{-k y}+\Phi_{0}^{2} e^{-k y} \arctan \left(e^{k y}\right)\right. \\
& \left.-\frac{\Phi_{0}^{2} e^{2 k y}\left(e^{2 k y}-1\right)}{3\left(e^{2 k y}+1\right)^{2}}\right] .
\end{aligned}
$$

Evaluating the above integral, we obtain the result 


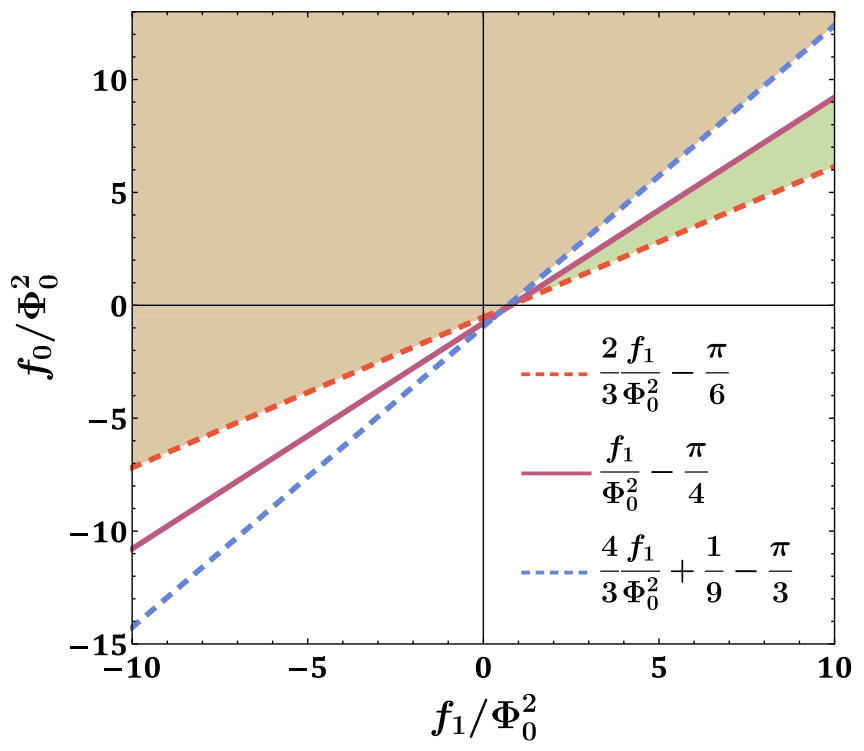

FIG. 18. Parameter space of the quantities $f_{0} / \Phi_{0}^{2}$ and $f_{1} / \Phi_{0}^{2}$. The curves corresponding to the expressions on the right-hand sides of the inequalities (8.7), (8.10), and (8.13) are depicted as well.

$$
\frac{1}{\kappa_{4}^{2}}=\frac{M_{P l}^{2}}{8 \pi}=\frac{f_{0}}{k}-\frac{2}{3} \frac{f_{1}}{k}+\frac{\pi}{6} \frac{\Phi_{0}^{2}}{k} .
$$

Since it is imperative to have a positive four-dimensional gravitational constant, we must satisfy also the following constraint:

$$
\frac{f_{0}}{\Phi_{0}^{2}}>\frac{2}{3} \frac{f_{1}}{\Phi_{0}^{2}}-\frac{\pi}{6} .
$$

In Fig. 18, we present the parameter space of the quantities $f_{0} / \Phi_{0}^{2}$ and $f_{1} / \Phi_{0}^{2}$, in an attempt to satisfy simultaneously the inequalities (8.7), (8.10), and (8.13). As it is clear, there is again no point where all three inequalities can be satisfied. The green area defines the part of the parameter space where $M_{P l}^{2}$ is positive and the weak energy conditions are satisfied by the bulk matter close to our brane, while the brown area defines the part of the parameter space where both the effective gravitational constant and the total energy density of the brane are positive.

\section{DISCUSSION AND CONCLUSIONS}

In the context of this work, we have investigated the emergence of braneworld solutions in the framework of a general scalar-tensor theory where the scalar field is nonminimally coupled to the five-dimensional Ricci scalar. In the bulk, these solutions are characterized by a RandallSundrum type, exponentially decaying warp factor, and a $y$ dependent scalar field with a bulk potential. On the brane, the space-time takes in general the form of a
Schwarzschild-(anti-)de Sitter solution. The present work completes our previous two analyses [69,73], where the cases of a de Sitter and an anti-de Sitter brane were considered, and focuses on the case of a flat, Minkowski brane with $\Lambda=0$. The complete five-dimensional solution for the gravitational background in this case may describe either a nonhomogeneous black string, when the metric parameter $M$ is nonzero, or a regular anti-de Sitter spacetime, when $M=0$.

The above features characterize our solutions irrespectively of the form of the coupling function between the bulk scalar field and the five-dimensional Ricci scalar. In this work, we have performed a comprehensive study of the types of braneworld solutions that emerge in the context of this theory by considering a plethora of forms of the coupling function, all supported by physical arguments regarding the reality and finiteness of its value everywhere in the bulk. We have thus considered the cases of a linear and quadratic coupling function in terms of the scalar field $\Phi$, but also an inverse-power and a linear-exponential form in terms of the $y$ coordinate. From a different perspective, we also considered given forms for the scalar field which again satisfied the finiteness condition, namely a doubleexponential and a hyperbolic-tangent form in terms of the $y$ coordinate, and determined subsequently the form of the coupling function. In all cases, the profile of the coupling function remains finite along the fifth coordinate as expected, reducing either to zero or to a constant value far away from our brane-in both cases, the coupling between the scalar field and the bulk Ricci scalar becomes trivial and as a result the scalar-tensor theory naturally reduces to a purely gravitational theory at large distances. Gravity by itself is also localized due to the exponentially decaying warp factor.

In each case, we have also determined in an analytical way the corresponding solutions for the profiles of the scalar field and scalar bulk potential. These also remain finite over the entire bulk for every solution found, and their behavior resembles the one of the coupling function reducing either to zero or to a constant value away from our brane. Depending on the values of the parameters of the solutions, the bulk potential in particular could adopt a variety of forms being nontrivial close to our brane and reducing to a constant, positive or negative, value at asymptotic infinity. What was of particular importance is the fact that the Randall-Sundrum-type, exponentially decaying warp factor is supported independently of the presence of the negative bulk cosmological constant $\Lambda_{5}$, which is usually introduced in an ad hoc way in braneworld models.

The case of a zero effective cosmological constant, studied in the context of this work, allowed for the maximum flexibility regarding the form and characteristics of the coupling function, when compared to the cases of a positive or negative effective cosmological constant 
$[69,73]$. For $\Lambda>0$, the coupling function had to be negative-definite at large distances from our brane, while for $\Lambda<0$, a fast localized profile was necessary in order to avoid an ill-defined behavior for the scalar field at the bulk boundaries. For $\Lambda=0$, though, no such requirements are necessary. In order, however, to derive physically acceptable braneworld solutions, we have imposed three additional conditions: the positivity of the effective gravitational constant $\kappa_{4}^{2}$ on our brane defined as

$$
\frac{1}{\kappa_{4}^{2}} \equiv 2 \int_{0}^{\infty} d y e^{-2 k y} f(y),
$$

the positivity of the total energy density of our brane, which follows from the junction condition (3.10) and may be rewritten as

$$
\sigma+V_{b}=6 k f(0)-2 f^{\prime}(0),
$$

and the validity of the weak energy conditions by the bulk matter in the vicinity of our brane; the latter, using Eqs. (3.6)-(3.8), may be expressed as

$$
f(0)<0 .
$$

In each solution found, we have thus performed a careful study of the effective theory on the brane, the junction conditions introduced by the presence of the brane, and the profiles of the energy density and pressure of the bulk matter. Subsequently, we conducted a thorough investigation of the corresponding parameter space in order to deduce whether it is possible to simultaneously satisfy all three aforementioned conditions.

We have found that, for all solutions presented, this is not possible. The aforementioned three constraints are not a priori incompatible: Eq. (9.3) constrains the value of the coupling function $f(y)$ at the location of the brane, Eq. (9.2) dictates that its first derivative must be also negative and decreasing fast at the same point, while Eq. (9.1) imposes a constraint on its integral over the entire bulk. Note that if we had demanded the validity of the weak energy condition everywhere in the bulk, i.e., $\rho(y)>0$, that would imply $f(y)<0$, for $\forall y$. This would be in obvious contradiction with the positivity of the effective gravitational constant through Eq. (9.1). Demanding the validity of the weak energy condition only at the vicinity of our brane, as in Eq. (9.3), allows the coupling function to be negative close to our brane and become positive at some distance off it, so that the integral in Eq. (9.1) turns out to be positive-definite. That was indeed realized for some of our solutions but the parameter space corresponding to those solutions was always severely restricted. Imposing the third constraint (9.2) on the value of $f^{\prime}(0)$, on top of the previous two constraints, in an attempt to make the energy density of the brane also positive, we were led to contradictions for all the analytical solutions we have found.

These contradictions are translated to the absence of a single point in the parameter space in which all the above constraints can be simultaneously satisfied. In contrast, relaxing the weak energy condition, which involves bulk quantities, and demanding instead the validity of Eqs. (9.1) and (9.2), which are relevant for the four-dimensional observer on the brane, has led to a plethora of analytic solutions with an extended parameter space. The question of whether a solution satisfying all three constraints could be constructed, either analytically or numerically, naturally emerges, and could be pursued in a future work. That solution, however, would have to be not only a mathematically consistent solution of the set of field equations satisfying the constraints (9.1)-(9.3) but to be also characterized by a physically acceptable behavior throughout the bulk - the analytical solutions presented in this work were carefully constructed in order to have a physically acceptable behavior regarding the profiles of the scalar field, its coupling function, and potential throughout the bulk.

The question of the stability of the solutions found in this work is also an important one. The presence of the scalar field, which is nonminimally coupled to gravity in the context of our theory, considerably complicates the stability analysis of the solutions found. Such an analysis will inevitably involve a coupled system of gravitational and scalar-field equations with the particular form of the coupling function, characterizing each solution, playing perhaps a decisive role in the outcome of the analysis. We note that, in all solutions presented in this work, the coupling function becomes trivial at large distances from the brane and the scalar field acquires a constant value. As a result, the nonminimally coupled scalar-tensor theory reduces there to a pure gravitational theory. Thus, at large distances from the brane, our solutions reduce to the blackstring solutions derived in [14] and are shown to be unstable in [55]. However, the nontrivial configurations of both the coupling function and the scalar field as we approach the brane may significantly alter the stability behavior of our solutions compared to that of the black string of [14]. It is quite likely that each of the obtained solutions has its own stability behavior under perturbations, and their future study may provide valuable restrictions on the exact form of the coupling function, bulk potential, and profile of the scalar field itself.

In conclusion, the well-known generalized gravitational theory of a scalar field nonminimally coupled to the Ricci scalar admits, upon embedded in a five-dimensional braneworld context, a variety of solutions with a number of attractive features, such as the support of an exponentially decaying warp factor, and thus of graviton localization, without the need for a negative bulk cosmological constant. In the particular case of $\Lambda=0$ studied here, this is always 
supplemented by a regular scalar field, a finite coupling function, which becomes naturally trivial at the outskirts of the bulk, a physically acceptable brane with a positive total energy density, and a robust effective four-dimensional theory on our brane.

\section{ACKNOWLEDGMENTS}

The research of T. N. was cofinanced by Greece and the European Union (European Social Fund- ESF) through the operational program "Human Resources Development, Education and Lifelong Learning" in the context of the project "Strengthening Human Resources Research Potential via Doctorate Research-2nd Cycle" (MIS-5000432), implemented by the State Scholarships Foundation (IKY). The research of N. P. was implemented under the "Strengthening Post-Doctoral Research" scholarship program (Grant No. 2016-050-0503-7626) by the Hellenic State Scholarships Foundation as part of the operational program "Human Resources Development Program, Education and Lifelong Learning," cofinanced by the European Social Fund-ESF and the Greek government.

\section{APPENDIX A: RESTRICTIONS ON THE ALLOWED VALUES OF THE PARAMETER $\mu$ IN QUADRATIC CASE}

We shall now determine the range of values for the parameter $\mu$ in the case of the quadratic coupling function (4.1). The allowed values of $\mu$ will be obtained by demanding that the scalar field (4.4) remains real and finite, and depend primarily on the assumed value of the parameter $\lambda$. In what follows, we will consider in detail every possible case:

(i) $\lambda>0$ :

Using Eq. (4.4) we get

$$
\lim _{y \rightarrow+\infty} \Phi(y)=\frac{\Phi_{0}}{2 \lambda}\left(\xi \mu^{\frac{2 \lambda}{1+4 \lambda}}-1\right) .
$$

Thus, demanding the functions $\Phi(y), f(y)$ to be real valued in their whole domain, it is necessary to have $\mu \geq 0$.

(ii) $\lambda \in\left(-\frac{1}{4}, 0\right) \wedge \frac{2 \lambda}{1+4 \lambda} \neq n, n \in \mathbb{Z}^{<}$:

In this case $\frac{2 \lambda}{1+4 \lambda}$ is a negative rational number.

Hence, one may write

$\lim _{y \rightarrow+\infty} \Phi(y)=\frac{\Phi_{0}}{2 \lambda}\left(\xi \mu^{\frac{2 \lambda}{1+4 \lambda}}-1\right)=-\frac{\Phi_{0}}{2|\lambda|}\left[1-\xi\left(\frac{1}{\mu}\right)^{\left|\frac{2 \lambda}{1+4 \lambda}\right|}\right]$.

Therefore, in order to avoid having a complex scalar field we should demand $\mu>0$.

(iii) $\lambda \in\left(-\frac{1}{4}, 0\right) \wedge \frac{2 \lambda}{1+4 \lambda}=n, n \in \mathbb{Z}^{<}$:

In this case we have $\frac{2 \lambda}{1+4 \lambda}=n$ or $\lambda=\frac{n}{2(1-2 n)}$. Thus, one may write

$$
\begin{aligned}
\Phi(y) & =\frac{\Phi_{0}}{2 \lambda}\left[\xi\left(\mu+e^{-k y}\right)^{\frac{2 \lambda}{1+4 \lambda}}-1\right] \\
& =\frac{\Phi_{0}(1-2 n)}{n}\left[\xi\left(\frac{1}{\mu+e^{-k y}}\right)^{|n|}-1\right] .
\end{aligned}
$$

It is clear that the parameter $\mu$ is allowed to take negative values. However, we should not allow values in the range $[-1,0]$, because then at $y_{0}=$ $-\frac{1}{k} \ln (-\mu)>0$ we would encounter infinities regarding both the scalar field and the coupling function in a finite distance away from the brane. Thus, $\mu \in(-\infty,-1) \cup(0, \infty)$.

(iv) $\lambda=-\frac{1}{4}$ :

In this particular case it is obvious from Eqs. (4.4) and (4.5) that the parameter $\mu$ is allowed to take any value in the set of the real numbers except zero. Thus, $\mu \in(-\infty, 0) \cup(0,+\infty)$.

(v) $\lambda<-\frac{1}{4} \wedge \frac{2 \lambda}{1+4 \lambda} \neq n, n \in \mathbb{Z}^{>}$:

In this case $\frac{2 \lambda}{1+4 \lambda}$ is a positive rational number. Thus, we have

$$
\lim _{y \rightarrow+\infty} \Phi(y)=\frac{\Phi_{0}}{2 \lambda}\left(\xi \mu^{\frac{2 \lambda}{1+4 \lambda}}-1\right) .
$$

Therefore, $\mu \geq 0$ to avoid a complex-valued scalar field.

(vi) $\lambda<-\frac{1}{4} \wedge \frac{2 \lambda}{1+4 \lambda}=n, n \in \mathbb{Z}^{>}$:

In this case, it is $\frac{2 \lambda}{1+4 \lambda}=n$ and $\lambda=\frac{n}{2(1-2 n)}$. Thus, from Eq. (4.4) we have

$$
\begin{aligned}
\Phi(y) & =\frac{\Phi_{0}}{2 \lambda}\left[\xi\left(\mu+e^{-k y}\right)^{\frac{2 \lambda}{1+4 \lambda}}-1\right] \\
& =\frac{\Phi_{0}(1-2 n)}{n}\left[\xi\left(\mu+e^{-k y}\right)^{n}-1\right],
\end{aligned}
$$

which allows $\mu$ to take values in the whole set of the real number: $\mu \in \mathbb{R}$.

The aforementioned results are summarized in Table I.

\section{APPENDIX B: THE UPPER AND LOWER INCOMPLETE GAMMA FUNCTIONS}

The upper incomplete gamma function $\Gamma(s, x)$ is defined as follows:

$$
\Gamma(s, x) \equiv \int_{x}^{\infty} d t t^{s-1} e^{-t}=\Gamma(s)-\gamma(s, x),
$$

where

$$
\gamma(s, x) \equiv \int_{0}^{x} d t t^{s-1} e^{-t}
$$

is the lower incomplete gamma function. Both upper and lower incomplete gamma functions, as defined above, are 
valid for real and positive $s$ and $x$. However, both functions can be extended for almost all combinations of complex $s$ and $x$. One can show that, for all complex $s$ and $z$, the lower incomplete gamma function can be expanded in the following power series:

$$
\gamma(s, z)=z^{s} \Gamma(s) e^{-z} \sum_{k=0}^{\infty} \frac{z^{k}}{\Gamma(s+k+1)} .
$$

Locally, the sum in the right-hand side of the previous relation converges uniformly for all $s \in \mathbb{C}$ and $z \in \mathbb{C}$. Using the relation $\Gamma(s, z)=\Gamma(s)-\gamma(s, z)$ we obtain the values of the upper incomplete gamma function for complex $s$ and $z$, but only for the points $(s, z)$ in which the righthand side exists. The numerical value of the upper incomplete gamma function can be given by the following expressions:

$$
\Gamma(s, x)=\left\{\begin{array}{ll}
\Gamma(s)-x^{s} \Gamma(s) e^{-x} \sum_{k=0}^{\infty} \frac{x^{k}}{\Gamma(s+k+1)}, & s \neq-n, n \in \mathbb{Z}^{>} \\
-\gamma-\ln (x)-\sum_{k=1}^{\infty} \frac{(-x)^{k}}{k(k !)}, & s=0 \\
\frac{1}{n !}\left[\frac{e^{-x}}{x^{n}} \sum_{k=0}^{n-1}(-x)^{k}(n-k-1) !+(-1)^{n} \Gamma(0, x)\right], & s=-n, n \in \mathbb{Z}^{>}
\end{array}\right\},
$$

where $\gamma$ is the Euler-Mascheroni constant. In our case, for $s=1-\lambda$, namely $s \in(-\infty, 1)$ and $x=2 k y_{0}>0$, we obtain the expressions

$$
\Gamma\left(1-\lambda, 2 k y_{0}\right)=\left\{\begin{array}{ll}
\Gamma(1-\lambda)\left[1-\left(2 k y_{0}\right)^{1-\lambda} e^{2 k y_{0}} \sum_{m=0}^{\infty} \frac{\left(2 k y_{0}\right)^{m}}{\Gamma(2+m-\lambda)}\right], & \lambda \neq n+1, \\
-\gamma-\ln \left(2 k y_{0}\right)-\sum_{m=1}^{\infty} \frac{\left(-2 k y_{0}\right)^{m}}{m(m !)}, & n \in \mathbb{Z}^{>} \\
\frac{1}{n !}\left[\frac{e^{-2 k y_{0}}}{\left(2 k y_{0}\right)^{n}} \sum_{m=0}^{n-1}\left(-2 k y_{0}\right)^{m}(n-m-1) !+(-1)^{n} \Gamma\left(0,2 k y_{0}\right)\right], & \lambda=n+1, \\
& n \in \mathbb{Z}^{>}
\end{array}\right\} .
$$

[1] A. Einstein, Zur allgemeinen relativitätstheorie, Sitzungsber. Preuss. Akad. Wiss. Berlin (Math. Phys.) 1915, 778 (1915).

[2] A. Einstein, Die Feldgleichungen der gravitation, Sitzungsber. Preuss. Akad. Wiss. Berlin (Math. Phys.) 1915, 844 (1915).

[3] A. Einstein, Die Grundlage der allgemeinen Relativitätstheorie, Ann. Phys. (Berlin) 354, 769 (1916).

[4] T. Kaluza, Zum UnitÃtsproblem der physik, Sitzungsber. Preuss. Akad. Wiss. Berlin (Math. Phys.) 1921, 966 (1921) [Int. J. Mod. Phys. D 27, 1870001 (2018)].

[5] O. Klein, Quantentheorie und fünfdimensionale relativitätstheorie, Z. Phys. 37, 895 (1926).

[6] V. A. Rubakov and M. E. Shaposhnikov, Do we live inside a domain wall?, Phys. Lett. 125B, 136 (1983).

[7] K. Akama, An early proposal of "Brane World", Lect. Notes Phys. 176, 267 (1982).

[8] N. Arkani-Hamed, S. Dimopoulos, and G. R. Dvali, The hierarchy problem and new dimensions at a millimeter, Phys. Lett. B 429, 263 (1998).

[9] N. Arkani-Hamed, S. Dimopoulos, and G. R. Dvali, Phenomenology, astrophysics and cosmology of theories with submillimeter dimensions and $\mathrm{TeV}$ scale quantum gravity, Phys. Rev. D 59, 086004 (1999).
[10] I. Antoniadis, N. Arkani-Hamed, S. Dimopoulos, and G. R. Dvali, New dimensions at a millimeter to a Fermi and superstrings at a TeV, Phys. Lett. B 436, 257 (1998).

[11] L. Randall and R. Sundrum, Large Mass Hierarchy from a Small Extra Dimension, Phys. Rev. Lett. 83, 3370 (1999).

[12] L. Randall and R. Sundrum, Alternative to Compactification, Phys. Rev. Lett. 83, 4690 (1999).

[13] R. Emparan and H. S. Reall, Black holes in higher dimensions, Living Rev. Relativity 11, 6 (2008).

[14] A. Chamblin, S. W. Hawking, and H. S. Reall, Brane world black holes, Phys. Rev. D 61, 065007 (2000).

[15] R. Emparan, G. T. Horowitz, and R. C. Myers, Exact description of black holes on branes, J. High Energy Phys. 01 (2000) 007.

[16] R. Emparan, G. T. Horowitz, and R. C. Myers, Exact description of black holes on branes. 2. Comparison with BTZ black holes and black strings, J. High Energy Phys. 01 (2000) 021.

[17] N. Dadhich, R. Maartens, P. Papadopoulos, and V. Rezania, Black holes on the brane, Phys. Lett. B 487, 1 (2000).

[18] P. Kanti and K. Tamvakis, Quest for localized 4-D black holes in brane worlds, Phys. Rev. D 65, 084010 (2002). 
[19] G. Kofinas, E. Papantonopoulos, and V. Zamarias, Black hole solutions in brane worlds with induced gravity, Phys. Rev. D 66, 104028 (2002).

[20] R. Casadio, A. Fabbri, and L. Mazzacurati, New black holes in the brane world?, Phys. Rev. D 65, 084040 (2002).

[21] V. P. Frolov, M. Snajdr, and D. Stojkovic, Interaction of a brane with a moving bulk black hole, Phys. Rev. D 68, 044002 (2003).

[22] P. Kanti, I. Olasagasti, and K. Tamvakis, Quest for localized 4-D black holes in brane worlds. 2. Removing the bulk singularities, Phys. Rev. D 68, 124001 (2003).

[23] G. Kofinas and E. Papantonopoulos, Gravitational collapse in brane world models with curvature corrections, J. Cosmol. Astropart. Phys. 12 (2004) 011.

[24] D. Karasik, C. Sahabandu, P. Suranyi, and L. C. R. Wijewardhana, Small black holes on branes: Is the horizon regular or singular?, Phys. Rev. D 70, 064007 (2004).

[25] C. Galfard, C. Germani, and A. Ishibashi, Asymptotically AdS brane black holes, Phys. Rev. D 73, 064014 (2006).

[26] S. Creek, R. Gregory, P. Kanti, and B. Mistry, Braneworld stars and black holes, Classical Quantum Gravity 23, 6633 (2006).

[27] B. Cuadros-Melgar, E. Papantonopoulos, M. Tsoukalas, and V. Zamarias, BTZ-like String on Codimension-2 Braneworlds in the Thin Brane Limit, Phys. Rev. Lett. 100, 221601 (2008).

[28] M. Anber and L. Sorbo, New exact solutions on the RandallSundrum 2-brane: Lumps of dark radiation and accelerated black holes, J. High Energy Phys. 07 (2008) 098.

[29] M. Heydari-Fard and H. R. Sepangi, Spherically symmetric solutions and gravitational collapse in braneworlds, J. Cosmol. Astropart. Phys. 02 (2009) 029.

[30] R. Casadio and J. Ovalle, Brane-world stars and (microscopic) black holes, Phys. Lett. B 715, 251 (2012).

[31] P. Kanti, N. Pappas, and K. Zuleta, On the localization of four-dimensional brane-world black holes, Classical Quantum Gravity 30, 235017 (2013).

[32] J. Ovalle and F. Linares, Tolman IV solution in the Randall-Sundrum braneworld, Phys. Rev. D 88, 104026 (2013).

[33] T. Harko and M. J. Lake, Null fluid collapse in brane world models, Phys. Rev. D 89, 064038 (2014).

[34] J. Ovalle, L. Ã. Gergely, and R. Casadio, Brane-world stars with a solid crust and vacuum exterior, Classical Quantum Gravity 32, 045015 (2015).

[35] A. M. Kuerten and R. da Rocha, Probing topologically charged black holes on brane worlds in $f(\mathrm{R})$ bulk, Gen. Relativ. Gravit. 48, 90 (2016).

[36] A. Herrera-Aguilar, A. M. Kuerten, and R. da Rocha, Regular bulk solutions in brane-worlds with inhomogeneous dust and generalized dark radiation, Adv. High Energy Phys. 2015, 1 (2015).

[37] R. Casadio, J. Ovalle, and R. da Rocha, The minimal geometric deformation approach extended, Classical Quantum Gravity 32, 215020 (2015).

[38] P. Kanti, N. Pappas, and T. Pappas, On the localization of four-dimensional brane-world black holes: II. The general case, Classical Quantum Gravity 33, 015003 (2016).

[39] R. Maartens and K. Koyama, Brane-World gravity, Living Rev. Relativity 13, 5 (2010).
[40] A. S. Majumdar and N. Mukherjee, Braneworld black holes in cosmology and astrophysics, Int. J. Mod. Phys. D 14, 1095 (2005).

[41] R. Gregory, Braneworld black holes, Lect. Notes Phys. 769, 259 (2009).

[42] P. Kanti, Brane-world black holes, J. Phys. Conf. Ser. 189, 012020 (2009).

[43] P. Kanti, Footprints of higher-dimensional decaying black holes, Rom. J. Phys. 57, 879 (2012).

[44] P. Kanti and E. Winstanley, Hawking radiation from higherdimensional black holes, Fundam. Theor. Phys. 178, 229 (2015).

[45] N. D. Pappas, The black hole challenge in Randall-Sundrum II model, arXiv:1409.0817.

[46] T. Nakas, Searching for localized black-hole solutions in Brane-world models, Master's thesis, Ioannina University, 2017.

[47] H. Kudoh, T. Tanaka, and T. Nakamura, Small localized black holes in brane world: Formulation and numerical method, Phys. Rev. D 68, 024035 (2003).

[48] H. Kudoh, Six-dimensional localized black holes: Numerical solutions, Phys. Rev. D 69, 104019 (2004); Erratum, Phys. Rev. D 70, 029901 (2004).

[49] N. Tanahashi and T. Tanaka, Time-symmetric initial data of large brane-localized black hole in RS-II model, J. High Energy Phys. 03 (2008) 041.

[50] B. Kleihaus, J. Kunz, E. Radu, and D. Senkbeil, Electric charge on the brane?, Phys. Rev. D 83, 104050 (2011).

[51] P. Figueras and T. Wiseman, Gravity and Large Black Holes in Randall-Sundrum II Braneworlds, Phys. Rev. Lett. 107, 081101 (2011).

[52] S. Abdolrahimi, C. Cattoen, D. N. Page, and S. YaghoobpourTari, Large Randall-Sundrum II black holes, Phys. Lett. B 720, 405 (2013).

[53] S. Abdolrahimi, C. CattoÃn, D. N. Page, and S. YaghoobpourTari, Spectral methods in general relativity and large RandallSundrum II black holes, J. Cosmol. Astropart. Phys. 06 (2013) 039.

[54] R. Gregory and R. Laflamme, Black Strings and p-Branes are Unstable, Phys. Rev. Lett. 70, 2837 (1993).

[55] R. Gregory, Black string instabilities in anti-de Sitter space, Classical Quantum Gravity 17, L125 (2000).

[56] S. S. Gubser, On nonuniform black branes, Classical Quantum Gravity 19, 4825 (2002).

[57] T. Wiseman, Static axisymmetric vacuum solutions and nonuniform black strings, Classical Quantum Gravity 20, 1137 (2003).

[58] H. Kudoh and T. Wiseman, Connecting Black Holes and Black Strings, Phys. Rev. Lett. 94, 161102 (2005).

[59] E. Sorkin, Critical Dimension in the Black String Phase Transition, Phys. Rev. Lett. 93, 031601 (2004).

[60] E. Sorkin, Nonuniform black strings in various dimensions, Phys. Rev. D 74, 104027 (2006).

[61] B. Kleihaus, J. Kunz, and E. Radu, New nonuniform black string solutions, J. High Energy Phys. 06 (2006) 016.

[62] M. Headrick, S. Kitchen, and T. Wiseman, A new approach to static numerical relativity, and its application to KaluzaKlein black holes, Classical Quantum Gravity 27, 035002 (2010). 
[63] C. Bogdanos, C. Charmousis, B. Gouteraux, and R. Zegers, Einstein-Gauss-Bonnet metrics: Black holes, black strings and a staticity theorem, J. High Energy Phys. 10 (2009) 037.

[64] C. Charmousis, T. Kolyvaris, and E. Papantonopoulos, Charged C-metric with conformally coupled scalar field, Classical Quantum Gravity 26, 175012 (2009).

[65] P. Figueras, K. Murata, and H. S. Reall, Stable non-uniform black strings below the critical dimension, J. High Energy Phys. 11 (2012) 071.

[66] M. Kalisch and M. Ansorg, Pseudo-spectral construction of non-uniform black string solutions in five and six spacetime dimensions, Classical Quantum Gravity 33, 215005 (2016).

[67] R. Emparan, R. Luna, M. MartÃnez, R. Suzuki, and K. Tanabe, Phases and stability of non-uniform black strings, J. High Energy Phys. 05 (2018) 104.

[68] A. Cisterna and J. Oliva, Exact black strings and p-branes in general relativity, Classical Quantum Gravity 35, 035012 (2018).

[69] P. Kanti, T. Nakas, and N. Pappas, Antigravitating braneworld solutions for a de Sitter brane in scalar-tensor gravity, Phys. Rev. D 98, 064025 (2018).

[70] A. Cisterna, C. Corral, and S. del Pino, Static and rotating black strings in dynamical Chern-Simons modified gravity, Eur. Phys. J. C 79, 400 (2019).

[71] A. Cisterna, L. Guajardo, and M. Hassaine, Axionic charged black branes with arbitrary scalar nonminimal coupling, Eur. Phys. J. C 79, 418 (2019); 79, 710 (2019).

[72] A. Cisterna, S. Fuenzalida, M. Lagos, and J. Oliva, Homogeneous black strings in Einstein-Gauss-Bonnet with Horndeski hair and beyond, Eur. Phys. J. C 78, 982 (2018).
[73] T. Nakas, N. Pappas, and P. Kanti, New black-string solutions for an anti-de Sitter brane in scalar-tensor gravity, Phys. Rev. D 99, 124040 (2019).

[74] S. Rezvanjou, R. Saffari, and M. Masoudi, Particle dynamics around the black string, arXiv:1707.02817.

[75] K. Farakos and P. Pasipoularides, Second Randall-Sundrum brane world scenario with a nonminimally coupled bulk scalar field, Phys. Rev. D 73, 084012 (2006).

[76] C. Bogdanos, A. Dimitriadis, and K. Tamvakis, Brane models with a Ricci-coupled scalar field, Phys. Rev. D 74, 045003 (2006).

[77] K. Farakos and P. Pasipoularides, Gauss-Bonnet gravity, brane world models, and nonminimal coupling, Phys. Rev. D 75, 024018 (2007).

[78] K. Farakos, G. Koutsoumbas, and P. Pasipoularides, Graviton localization and Newton's law for brane models with a nonminimally coupled bulk scalar field, Phys. Rev. D 76, 064025 (2007).

[79] S. Bhattacharya and S. R. Kousvos, Constraining the phantom braneworld model from cosmic structure sizes, Phys. Rev. D 96, 104006 (2017).

[80] S. Bhattacharya, S. R. Kousvos, S. Romanopoulos, and T. N. Tomaras, Cosmological screening and the phantom braneworld model, Eur. Phys. J. C 78, 637 (2018).

[81] P. Binetruy, C. Deffayet, and D. Langlois, Nonconventional cosmology from a brane universe, Nucl. Phys. B565, 269 (2000).

[82] A. Padilla and V. Sivanesan, Boundary terms and junction conditions for generalized scalar-tensor theories, J. High Energy Phys. 08 (2012) 122.

[83] M. Abramowitz and I. A. Stegun, Handbook of Mathematical Functions with Formulas, Graphs, and Mathematical Tables (Dover, New York, 1964). 